\title{
THE INVERSION OF THE LAPLACE INTEGRAL AND THE RELATED MOMENT PROBLEM*
}

\author{
BY \\ D. V. WIDDER \\ INTRODUCTION
}

Let $\alpha(t)$ be a complex function of the real variable $t$, of bounded variation in the interval $(0, R)$ for every positive $R$, and such that the integral

$$
f(x)=\int_{0}^{\infty} e^{-x t} d \alpha(t)
$$

converges for some complex value of $x$. It is then known that the integral will converge for all complex $x$ of greater real part and will consequently define a function, which we have denoted by $f(x)$, in a half-plane. By the inversion of the integral (1) we mean the determination of the function $\alpha(t)$ in terms of the function $f(x)$. There is one special case of (1) of particular interest, that in which $\alpha(t)$ is a step-function with jumps only at the integral points. Then

$$
f(x)=\sum_{n=0}^{\infty} a_{n} e^{-n x}
$$

In this case the problem of inversion reduces to the determination of the coefficients of the power series (2). If we set $z=e^{-x}$ in (2) we get

$$
F(z)=f(\log (1 / z))=\sum_{n=0}^{\infty} a_{n} z^{n}
$$

and there are two familiar determinations of the coefficients. One is in terms of a contour integral

$$
a_{n}=\frac{1}{2 \pi i} \int_{C} \frac{F(z)}{z^{n+1}} d z
$$

where the contour $C$ may be taken as a circle with center at $z=0$ and with any sufficiently small radius, the integration being in the positive sense. The other is

$$
a_{n}=F^{(n)}(0) / n ! .
$$

If we return to the function $f(x)$, the contour $C$ becomes a vertical line in

* Presented to the Society, December 29, 1932; received by the editors January 13, 1933, and in revised form in June, 1933. 
the $x$-plane. Likewise we should have

$$
a_{n}=\left.\lim \frac{1}{n !} \frac{d^{n}}{d z^{n}} f\left(\log \frac{1}{z}\right)\right|_{z=e^{-x}}
$$

as $x$ becomes infinite along the positive real axis.*

Reasoning from this special case, we should expect that for the general case (1) there would be two determinations of $\alpha(t)$, one in terms of a contour integral along a vertical line, the other in terms of the derivatives of $f(x)$ for large real positive values of $x$. The first of these is in fact very well known. For the special case in which

$$
\alpha(t)=\int_{0}^{t} \phi(u) d u
$$

and

$$
f(x)=\int_{0}^{\infty} e^{-x t} \phi(t) d t
$$

it was already known to Cauchy in the form

$$
\phi(t)=\frac{1}{2 \pi i} \int_{c-i \infty}^{c+i \infty} f(x) e^{x t} d x,
$$

where $c$ is a sufficiently large real constant and the path of integration is a vertical line. The general case (1) seems first to have been treated by $\mathrm{H}$. Hamburger. $\dagger$ It is remarkable that the second method, discovered the first in the special case (2), has received no attention until very recent times. It was apparently discovered first by W. P. Mason in his researches in electrical theory of 1929. No rigorous derivation of the inversion formula was published, however. In $1930 \mathrm{E}$. L. Post did obtain an inversion formula of the type in question for the special case in which the function $\alpha(t)$ has the form (3) with $\phi(u)$ a continuous function. It is the purpose of the present paper to obtain an inversion formula for the general integral (1).

By way of introducing this inversion operator let us consider first functions $f(x)$ which are analytic at infinity and which vanish there. That is, $f(x)$

* We could of course take the approach along any parallel line or along any curve proceeding indefinitely to the right.

$\dagger$ H. Hamburger, Über eine Riemannsche Formel aus der Theorie der Dirichletschen Reihen, Mathematische Zeitschrift, vol. 6 (1920), p. 6. See also D. V. Widder, A generalization of Dirichlet's series and of Laplace's integrals by means of a Stieltjes integral, these Transactions, vol. 31 (1929), p. 708. We shall refer to this latter paper as $I$.

$\ddagger$ E. L. Post, Generalized differentiation, these Transactions, vol. 32 (1930), p. 772. 
can be represented for $|x|$ sufficiently large by a convergent power series in $1 / x$,

$$
f(x)=\sum_{n=0}^{\infty} \frac{b_{n}}{x^{n+1}}
$$

If we note that

$$
\frac{1}{x^{n+1}}=\int_{0}^{\infty} e^{-x t} \frac{t^{n}}{n !} d t
$$

for all $x$ whose real part is positive, we see that

$$
\begin{aligned}
& f(x)=\int_{0}^{\infty} e^{-x t} \phi(t) d t, \\
& \phi(t)=\sum_{n=0}^{\infty} \frac{b_{n} t^{n}}{n !} .
\end{aligned}
$$

Now introduce the operator

$$
L_{k, t}[f(x)]=\frac{(-1)^{k}}{k !} f^{(k)}\left(\frac{k}{t}\right)\left(\frac{k}{t}\right)^{k+1} .
$$

For the series (5),

$$
L_{k, t}[f(x)]=\sum_{n=0}^{\infty} \frac{(n+k) !}{n ! k !} b_{n}\left(\frac{t}{k}\right)^{n} .
$$

As $k$ becomes infinite,

$$
\lim _{k \rightarrow \infty} \frac{(n+k) !}{k ! k^{n}}=1 \quad(n=0,1, \cdots),
$$

so that, if it were permissible to interchange summation and limit signs, we should have

$$
\lim _{k \rightarrow \infty} L_{k, t}[f(x)]=\sum_{n=0}^{\infty} b_{n} \frac{t^{n}}{n !}=\phi(t) .
$$

This leads us to introduce an operator $L_{t}[f(x)]$ by the equation

$$
L_{t}[f(x)]=\lim _{k \rightarrow \infty} L_{k, t}[f(x)] \text {. }
$$

We could easily justify the formal steps taken above and show rigorously that

$$
L_{t}[f(x)]=\phi(t) \quad(0<t<\infty) .
$$

However, our purpose for the moment is merely to provide a heuristic ap- 
proach. Much more general cases, in which the method of series will not be applicable, will be treated later.

We may now see what sort of operator will apply to the Stieltjes integral (1) by performing an integration by parts,

$$
f(x)=x \int_{0}^{\infty} e^{-x t} \alpha(t) d t .
$$

If we apply the operator $L_{t}$ to the function $f(x) / x$, we expect, guided by the above formal work, to obtain the function $\alpha(t)$. On the other hand we can show, as we do in $\S 3$, that

$$
L_{k, t}[f(x) / x]=f(\infty)+(-1)^{k+1} \int_{k / t}^{\infty} \frac{u^{k}}{k !} f^{(k+1)}(u) d u .
$$

We are thus led to introduce a second operator $S_{t}$ by the equations

$$
\begin{aligned}
S_{k, t}[f(x)] & =f(\infty)+(-1)^{k+1} \int_{k / t}^{\infty} \frac{u^{k}}{k !} f^{(k+1)}(u) d u, \\
S_{t}[f(x)] & =\lim _{k \rightarrow \infty} S_{k, t}[f(x)] .
\end{aligned}
$$

We shall show in $\$ 3$ that our conjecture is verified, that for every convergent integral (1)

$$
S_{t}[f(x)]=\frac{\alpha(t+)+\alpha(t-)}{2} \quad(0<t<\infty)
$$

at least if a suitable constant is added to the function $\alpha(t)$ so as to make $\alpha(0)=0$. (This change in $\alpha(t)$ of course produces no change in $f(x)$.)

In $\$ 4$ we treat the most general integral (4) where the function $\phi(t)$ is integrable in the sense of Lebesgue, and we find that the operator $L_{t}$ inverts the integral for almost all positive values of $x$. The remaining sections of Part $I$ are devoted to further inversion formulas, it being always understood that the function $f(x)$ is known to have a representation (1). In Part II we drop this assumption and consider all functions $f(x)$ to which the operators $L$ and $S$ are applicable. Their very nature demands, of course, that the functions $f(x)$ must have derivatives of all orders and must have certain asymptotic properties as $x$ becomes positively infinite. We are thus able to develop necessary and sufficient conditions that a function $f(x)$ should have a representation (1). Among other results we prove a theorem of S. Bernstein to the effect that if $f(x)$ is completely monotonic,

$$
(-1)^{k} f^{(k)}(x) \geqq 0
$$$$
(k=0,1, \cdots),
$$ 
it must have the representation (1) with $\alpha(t)$ a non-decreasing function. By use of our operator $S$ this result becomes almost self-evident. For, if (7) holds, $S_{k, t}[f(x)]$ is clearly a non-decreasing function of $t$. The same must be true of $S_{t}[f(x)]$ if this function exists. The existence of this limit of course requires proof.

In Part III we take up several applications. First we discuss the zeros of a function $f(x)$ represented in the form (1). To make our results more descriptive let us consider here the case (4) where $\phi(t)$ is real and continuous. We are able to show that if $\phi(t)$ has just $n$ changes of sign in $(0, \infty)$ then $f^{(k)}(x)$ will have exactly the same number in the interval of convergence of (4) for all $k$ sufficiently large. We are even able to compute the coordinates of the zeros of $\phi(t)$ in terms of those of $f^{(k)}(x)$. Thus if the zeros of $\phi(t)$ are at the points $t_{1}, t_{2}, \cdots, t_{n}$, where

$$
0<t_{1}<t_{2}<\cdots<t_{n}
$$

and if those of $f^{(k)}(x)$ are at the points $x_{1, k}, x_{2, k}, \cdots, x_{n, k}$, where

then

$$
x_{1, k}>x_{2, k}>\cdots>x_{n, k},
$$

$$
\lim _{k \rightarrow \infty} \frac{k}{x_{i, k}}=t_{i} . \quad(i=1,2, \cdots) .
$$

In an article in the Proceedings of the National Academy of Sciences* we announced this result with the restriction that $\phi(t)$ should approach a limit as $t$ becomes infinite, observing that the condition was probably redundant. J. Karamata $\dagger$ in a subsequent note of the same Proceedings removed this restriction but imposed another. In the present paper we remove all conditions of the type, demanding only that the integral (1) should converge, a condition imposed by the nature of the problem. We do not even demand that $\alpha(t)$ or $\phi(t)$ should be continuous.

In Part IV we treat the complex case. The integral is taken in the form (4), and the function $\phi(t)$ is supposed analytic in the half-plane for which the real part of $t$ is positive and of such a nature that the integral (4) converges when the path of integration is the positive real axis. We are then able to show that

$$
L_{t}[f(x)]=\phi(t)
$$

for all complex $t$ whose real part is positive. By use of this result we are able to treat also the complex zeros of $\phi(t)$ in terms of the complex zeros of $f^{(k)}(x)$.

*D. V. Widder, On the changes of sign of the derivatives of a function defined by a Laplace integral, Proceedings of the National Academy of Sciences, vol. 18 (1932), p. 112.

$\dagger \mathrm{J}$. Karamata, Remarks on a theorem of D. V. Widder, Proceedings of the National Academy of Sciences, vol. 18 (1932), p. 406. 
In Part $\mathrm{V}$ we take up a moment problem intimately related to the Laplace integral. If in (1) we allow $x$ to take on only positive integral values we have by an obvious change of variable

$$
f(n)=\mu_{n}=\int_{0}^{\infty} e^{-n t} d \alpha(t)=\int_{0}^{1} t^{n} d \beta(t) \quad(n=0,1,2, \cdots) .
$$

The determination of $\beta(t)$ in terms of the sequence $\left\{\mu_{n}\right\}$ is the moment problem of Hausdorff. Since the variable $n$ now runs through a discrete set of values we expect to get an operator applicable to the sequence $\left\{\mu_{n}\right\}$ as the operator $L$ was to the integral (4) by replacing the derivative of order $k$ by a difference of order $k$ and by replacing a $(k+1)$ th power of $x$ by a product $n(n+1) \cdots(n+k)$. Proceeding in this way we are led to define the operator $L_{t}\left\{\mu_{n}\right\}$ by the equations

$$
\begin{aligned}
L_{k, t}\left\{\mu_{n}\right\} & =\frac{(n+k+1) !}{n ! k !}(-1)^{k} \Delta^{k} \mu_{n}, . \quad n=\left[\frac{k t}{1-t}\right], \\
L_{t}\left\{\mu_{n}\right\} & =\lim _{k \rightarrow \infty} L_{k, t}\left\{\mu_{n}\right\} .
\end{aligned}
$$

Here $[u]$ means the greatest integer contained in $u$. We find in fact that this operator does invert the moment sequence (8) if $\beta(t)$ has the form (3) with $\phi(t)$ integrable in $(0,1)$. That is,

$$
L_{t}\left\{\mu_{n}\right\}=\phi(t)
$$

almost everywhere in $(0,1)$. In defining an operator $S$ which will be applicable to the general sequence (8) we again proceed by analogy replacing the integral sign by the summation sign and the derivatives by differences in (6). In this way we arrive at the operators

$$
\begin{aligned}
S_{k, t}\left\{\mu_{n}\right\} & =-\mu_{\infty}-\sum_{i=n+1}^{\infty} \frac{(i+k) !}{i ! k !}(-1)^{k+1} \Delta^{k+1} \mu_{i}, \\
S_{t}\left\{\mu_{n}\right\} & =\lim _{k \rightarrow \infty} S_{k, t}\left\{\mu_{n}\right\}, \quad n=\left[\frac{k t}{1-t}\right] .
\end{aligned}
$$

We then prove that

$$
S_{t}\left\{\mu_{n}\right\}=\frac{\beta(t+)+\beta(t-)}{2} \quad(0<t<1) .
$$

We then turn to sequences $\left\{\mu_{n}\right\}$ which are not known to be moment sequences and discuss the effect of the operators $L$ and $S$ on them. We are able 
to obtain necessary and sufficient conditions that a given sequence should be a moment sequence. In particular Hausdorff's theorem to the effect that every completely monotonic sequence,

$$
(-1)^{k} \Delta^{k} \mu_{n} \geqq 0 \quad(k=0,1, \cdots ; n=0,1, \cdots),
$$

has the form (8) with $\beta(t)$ non-decreasing is obtained by use of the moment operator $S_{t}\left\{\mu_{n}\right\}$ with the same ease as Bernstein's theorem was obtained by use of the integral operator $S_{t}[f(x)]$.

As a further application of the operators $L$ and $S$ a study is made of the changes of sign of a sequence $\left\{\mu_{n}\right\}$ as affected by the changes of trend in $\beta(t)$ or by the changes of sign in $\phi(t)$. Results analogous to these already mentioned for the Laplace integral are obtained, results which serve to generalize certain theorems of $\mathbf{M}$. Fekete. $\dagger$ In conclusion the complex case is treated. A slight modification of the operator $L$ is necessary to give it meaning for complex $t$. In the foregoing definition we merely replace $[k t /(1-t)]$ by $k t /(1-t)$ thus defining an operator which we denote by $L_{t}^{*}\left\{\mu_{n}\right\}$. We find that if $\phi(t)$ is analytic in a circle of unit diameter with center at $t=\frac{1}{2}$, then throughout that circle

$$
L_{t}^{*}\left\{\mu_{n}\right\}=\phi(t) .
$$

The method found to be most serviceable in the major part of this paper is the Laplace method of determining an asymptotic expression for an integral of the form

$$
\int[f(t)]^{k} \phi(t) d t
$$

when $k$ becomes infinite. In the last section of the paper we use a modification of this method due to Perron for the case in which the integrand is complex and the path of integration is in the complex plane.

\section{PART I}

\section{INVERSION FORMULAS}

1. The problem. In Part I we shall discuss functions $f(x)$ of the real variable $x$ which are known to be expressible by means of a Laplace-Stieltjes integral

$$
f(x)=\int_{0}^{\infty} e^{-x t} d \alpha(t)
$$

where the function $\alpha(t)$ is a real function of bounded variation in the interval

$† \mathrm{M}$. Fekete, Sur les changements de signe d'une fonction continue dans un intervalle, Paris Comptes Rendus, vol. 190 (1930), p. 1366. References to Fekete's earlier work on the subject are given in this article. 
$0 \leqq t \leqq R$ for every positive $R$, where $\alpha(0)=0$, and where the integral converges for some value of $x$. We shall obtain a formula for the determination of $\alpha(t)$ in terms of the values of $f(x)$ and its derivatives. It will appear that a knowledge of these values in a neighborhood of $x=+\infty$ will be sufficient. In particular if $\alpha(t)$ is the integral of a function $\phi(t)$ so that (1.1) becomes

$$
f(x)=\int_{0}^{\infty} e^{-x t} \phi(t) d t,
$$

we shall obtain a similar formula for $\phi(t)$.

2. A preliminary limit. In order to develop the inversion formula for (1.1) we find it useful to know the value of the following limit:

$$
\lim _{k \rightarrow \infty} e^{-k / t}\left[1+\frac{k}{l}+\frac{1}{2 !}\left(\frac{k}{t}\right)^{2}+\cdots+\frac{1}{k !}\left(\frac{k}{t}\right)^{k}\right]
$$

for all positive $t$. To show the existence of this limit and to obtain its value we first express it as a definite integral by use of Taylor's formula with exact remainder,

$f(x)=f(0)+f^{\prime}(0) x+f^{\prime \prime}(0) \frac{x^{2}}{2 !}+\cdots+f^{(k)}(0) \frac{x^{k}}{k !}+\int_{0}^{x} \frac{(x-u)^{k}}{k !} f^{(k+1)}(u) d u$.

For our purposes take $f(x)=e^{x}$ and replace $x$ by $k / t$. Then

$$
\begin{aligned}
e^{k / t}=1+\frac{k}{t}+\frac{1}{2 !}\left(\frac{k}{t}\right)^{2}+\cdots+\frac{1}{k !}\left(\frac{k}{t}\right)^{k}+\frac{1}{k !} \int_{0}^{k / t}\left(\frac{k}{t}-u\right)^{k} e^{u} d u, \\
e^{-k / t}\left[1+\frac{k}{t}+\frac{1}{2 !}\left(\frac{k}{t}\right)^{2}+\cdots+\frac{1}{k !}\left(\frac{k}{t}\right)^{k}\right] \\
=1-\frac{1}{k !} \int_{0}^{k / t}\left(\frac{k}{t}-u\right)^{k} e^{u-(k / t)} d u=1-\frac{1}{k !} \int_{0}^{k / t} u^{k} e^{-u} d u .
\end{aligned}
$$

If we set $u=k y$ this becomes

$$
H_{k}(t)=1-\frac{k^{k+1}}{k !} \int_{0}^{1 / t}\left[y e^{-\nu}\right]^{k} d y .
$$

We can show at once that $H_{k}(t)$ approaches 1 as $k$ becomes infinite for $t>1$. For, the function $y e^{-v}$ has a single maximum at $y=1$, and is consequently increasing for $y<1$, decreasing for $y>1$. For $t>1$ this maximum is outside the interval of integration. Hence

$$
\left|H_{k}(t)-1\right|<\frac{k^{k+1}}{k !} \frac{e^{-k / t}}{t^{k+1}}
$$


The right-hand side of this inequality approaches zero as $k$ becomes infinite, as one sees by use of Stirling's formula.

Next suppose that $t<1$. Since

$$
k !=\int_{0}^{\infty} e^{-u} u^{k} d u
$$

we may clearly obtain the following expression for $H_{k}(t)$ :

$$
H_{k}(t)=\frac{1}{k !} \int_{k / t}^{\infty} u^{k} e^{-u} d u=\frac{k^{k+1}}{k !} \int_{1 / t}^{\infty}\left[y e^{-y}\right]^{k} d y .
$$

If $t<1$, the function $y e^{-y}$ is a decreasing function for $y>1 / t$, so that

$$
\left|H_{k}(t)\right|<\left\lceil\left[\frac{1}{t} e^{-1 / t}\right]^{k-1} \frac{k^{k+1}}{k !} \int_{1 / t}^{\infty} y e^{-y} d y .\right.
$$

The right-hand side of this inequality approaches zero as $k$ becomes infinite, so that $H_{k}(t)$ must do so also.

It remains to treat the case $t=1$. We have

$$
H_{k}(1)=1-\frac{1}{k !} \int_{0}^{k} u^{k} \xi^{-u} d u,
$$

and by a direct application of Laplace's method* we see that

$$
\lim _{k \rightarrow \infty} H_{k}(1)=\frac{1}{2} \text {. }
$$

We have thus proved

Theorem 1. On setting

$$
H_{k}(t)=e^{-k / t}\left[1+\frac{k}{t}+\frac{1}{2 !}\left(\frac{k}{t}\right)^{2}+\cdots+\frac{1}{k !}\left(\frac{k}{t}\right)^{k}\right]
$$

we have

and

$$
H_{k}(t)=1-\int_{0}^{k / t} \frac{u^{k}}{k !} e^{-u} d u=\int_{k / t}^{\infty} \frac{u^{k}}{k !} e^{-u} d u,
$$

$$
\lim _{k \rightarrow \infty} H_{k}(t)=\left\{\begin{array}{l}
0 \text { if } 0<t<1 \\
\frac{1}{2} \text { if } t=1 \\
1 \text { if } 1<t<\infty .
\end{array}\right.
$$

If we set $g(t)=\lim _{k \rightarrow \infty} H_{k}(t)$, then

* G. P6lya and G. Szegö, Aufgaben und Lehrsätze aus der Analysis, vol. I, chapter 2, p. 80, problem 210. The result was known to Jacobi; cf. Gesammelte Werke, vol. 7, p. 213. 


$$
e^{-x}=\int_{0}^{\infty} e^{-x t} d g(t)
$$

so that Theorem 1 gives us an inversion formula for the integral (1.1) in this special case.

3. The inversion of the Laplace-Stieltjes integral. From the result of Theorem 1 we can now conjecture the inversion operator for the general integral (1.1). We introduce the following

Definition. An operator $S_{t}[f(x)]$ is defined by the equations

$$
\begin{aligned}
S_{k, t}[f(x)] & =f(\infty)+(-1)^{k+1} \int_{k / t}^{\infty} \frac{u^{k}}{k !} f^{(k+1)}(u) d u \quad(k=0,1,2, \cdots), \\
S_{t}[f(x)] & =\lim _{k \rightarrow \infty} S_{k, t}[f(x)] .
\end{aligned}
$$

For this operator to be applicable to a given function $f(x)$ it is necessary that each of the operations involved in the definition should be well defined for the function. Thus $f(x)$ must have continuous derivatives of all orders, must approach a finite limit as $x$ becomes infinite, the improper integrals (3.1) must converge, and the limit (3.2) must exist. The operator is clearly distributive. We shall show in this section that it is well defined if $f(x)$ has the representation (1.1) and that it serves to invert that integral. The result to be established is the following:

THEOREM 2. If the integral

$$
f(x)=\int_{0}^{\infty} \cdot e^{-x t} d \alpha(t) \quad(\alpha(0)=0)
$$

converges for $x>c$, then

$$
S_{t}[f(x)]=\frac{\alpha(t+)+\alpha(t-)}{2} \quad(t>0) .
$$

Since the given integral converges for $x>c$ we know that there exists a constant $M$ such that

$$
|\alpha(t)|<M e^{o t} \quad(0 \leqq t<\infty),
$$

where $g$ is a positive constant greater than $c .^{*}$ Hence, on integrating by parts, we obtain

* D. V. Widder, I, p. 703, Lemma 2. 


$$
\begin{aligned}
& f(x)=\lim _{t \rightarrow \infty}\left[e^{-x t} \alpha(t)\right]+x \int_{0}^{\infty} e^{-x t} \alpha(t) d t \quad(x>g), \\
& f(x)=x \int_{0}^{\infty} e^{-x t} \alpha(t) d t .
\end{aligned}
$$

Set $f(x) / x=F(x)$ and introduce a new operator $S$ by the

Definition. An operator $L_{t}[f(x)]$ is defined by the equations

$$
\begin{aligned}
L_{k, t}[f(x)] & =(-1)^{k} f^{(k)}(k / t)(k / t)^{k+1} / k ! \quad(k=0,1,2, \cdots), \\
L_{t}[f(x)] & =\lim _{k \rightarrow \infty} L_{k, t}[f(x)] .
\end{aligned}
$$

We shall show that this operation is well defined when applied to $F(x)$ and that the result of the operation is $[\alpha(t+)+\alpha(t-)] / 2$. If $x>g$ equation (3.5) gives us*

$$
F^{(k)}(x)=(-1)^{k} \int_{0}^{\infty} e^{-x t} t^{k} \alpha(t) d t
$$

Hence

$$
L_{k, t}[F(x)]^{\cdot}=\frac{1}{k !}\left(\frac{k}{t}\right)^{k+1} \int_{0}^{\infty} e^{-k, y / t} y^{k} \alpha(y) d y .
$$

Let $t_{0}$ be an arbitrary positive value of $t$ and make the transformation $u=y / t_{0}$. Then

$$
L_{k, t_{0}}[F(x)]=\frac{k^{k+1}}{k !} \int_{0}^{\infty} e^{-k u} u^{k} \alpha\left(t_{0} u\right) d u .
$$

By use of the function $g(t)$ of $\$ 2$ we now define the function

$$
\psi(u)=\left[\alpha\left(t_{0}+\right)-\alpha\left(t_{0}-\right)\right] g(u)+\alpha\left(t_{0}-\right) .
$$

This function has the properties

so that the function

$$
\begin{gathered}
\psi(1+)=\alpha\left(t_{0}+\right) \\
\psi(1-)=\alpha\left(t_{0}-\right) \\
\psi(1)=\frac{\alpha\left(t_{0}+\right)+\alpha\left(t_{0}-\right)}{2}
\end{gathered}
$$

$$
\phi(u)=\alpha\left(t_{0} u\right)-\psi(u)
$$

has the properties

* D. V. Widder, I, p. 702, Corollary 2. 


$$
\phi(1+)=\phi(1-)=0 \text {. }
$$

We wish to show that the difference

$$
L_{k, t_{0}}[F(x)]-\frac{\alpha\left(t_{0}+\right)+\alpha\left(t_{0}-\right.}{2}
$$

approaches zero with $1 / k$. But

$$
\begin{aligned}
& \frac{k^{k+1}}{k !} \int_{0}^{\infty} e^{-k u} u^{k} \psi(u) d u=\left[\alpha\left(t_{0}+\right)-\alpha\left(t_{0}-\right)\right] \frac{k^{k+1}}{k !} \int_{0}^{\infty} e^{-k u} u^{k} g(u) d u+\alpha\left(t_{0}-\right) \\
& \quad=\left[\alpha\left(t_{0}+\right)-\alpha\left(t_{0}-\right)\right] \frac{k^{k+1}}{k} \int_{1}^{\infty} e^{-k u} u^{k} d u+\alpha\left(t_{0}-\right) \\
& \quad=\left[\alpha\left(t_{0}+\right)-\alpha\left(t_{0}-\right)\right] H_{k}(1)+\alpha\left(t_{0}-\right) \rightarrow \frac{1}{2}\left[\alpha\left(t_{0}+\right)+\alpha\left(t_{0}-\right)\right](k \rightarrow \infty) .
\end{aligned}
$$

Hence

$$
L_{t_{0}}[F(x)]-\frac{\alpha\left(t_{0}+\right)+\alpha\left(t_{0}-\right)}{2}=\lim _{k \rightarrow \infty} \frac{k^{k+1}}{k !} \int_{0}^{\infty} e^{-k u} u^{k}\left[\alpha\left(t_{0} u\right)-\psi(u)\right] d u .
$$

By (3.6) we see that to an arbitrary positive quantity $\epsilon$ there corresponds a number $\eta$ such that

$$
|\phi(u)|<\epsilon / 3 \quad(0<|1-u|<\eta<1) .
$$

Now divide the interval of integration in the right-hand member of (3.7) into $(0,1-\eta),(1-\eta, 1+\eta),(1+\eta, \infty)$, the corresponding contributions being defined as $I_{1}, I_{2}$, and $I_{3}$ respectively. Then

$$
\left|I_{2}\right|<\frac{\epsilon}{3} \frac{k^{k+1}}{k !} \int_{1-\eta}^{1+\eta} e^{-k u} u^{k} d u<\frac{\epsilon}{3} \frac{k^{k+1}}{k !} \int_{0}^{\infty} e^{-k u} u^{k} d u=\frac{\epsilon}{3} .
$$

If we denote by $K$ an upper bound of $|\phi(u)|$ in the interval $(0,1)$, we have as in $\S 2$

$$
\left|I_{1}\right| \leqq \frac{k^{k+1}}{k !} K e^{-k(1-\eta)}(1-\eta)^{k}
$$

Since the right-hand side of this inequality approaches zero with $1 / k$ we can determine $k_{0}$ so large that

$$
\left|I_{1}\right|<\epsilon / 3
$$

Finally, since

$$
\begin{aligned}
& |\phi(u)| \leqq\left|\alpha\left(t_{0} u\right)\right|+|\psi(u)|, \\
& |\phi(u)| \leqq M e^{o t_{0} u}+3 M e^{t_{0}}<N e^{\gamma u} \quad(\gamma>0),
\end{aligned}
$$


we have

$$
\left|I_{3}\right|<\frac{k^{k+1}}{k !} e^{-(k-\lambda)(1+\eta)}(1+\eta)^{k-\lambda} \int_{1+\eta}^{\infty} e^{-\lambda u} u^{\lambda} N e^{\gamma u} d u .
$$

Here $\lambda$ is any fixed number greater than $\gamma$, so that the integral(3.9) converges. The right-hand side of (3.9) tends to zero with $1 / k$. Hence we can find $k_{1}$ greater than $k_{0}$ such that

$$
\left|I_{3}\right|<\epsilon / 3
$$

Then for $k>k_{1}$

and

$$
\left|L_{k, t_{0}}[F(x)]-\frac{\alpha\left(t_{0}+\right)+\alpha\left(t_{0}-\right)}{2}\right|<\epsilon
$$

$$
L_{t_{0}}[F(x)]=\frac{\alpha\left(t_{0}+\right)+\alpha\left(t_{0}-\right)}{2} .
$$

It will now be established that

$$
L_{k, t}[F(x)]=S_{k, t}[f(x)],
$$

and this will complete the proof of the theorem. It is not evident that the operator $S$ has a meaning as applied to $f(x)$. To show that it has we prove first that

(3.10) $(-1)^{n} f^{(n)}(x)=\int_{0}^{\infty} e^{-x t} t^{n} d \alpha(t)=o\left(x^{-n}\right) \quad(x \rightarrow \infty ; n=1,2, \cdots)$.

Indeed, if we set

$$
\beta(0)=0, \beta(t)=\int_{0}^{t}(-1)^{n} u^{n} d \omega(u) \quad(t>0),
$$

where

$$
\omega(0)=0, \omega(u)=\alpha(u)-\alpha(0+)
$$

we have

$$
\begin{aligned}
f^{(n)}(x) & =\int_{0}^{\infty} e^{-x t} d \beta(t) \\
& =x \int_{0}^{\infty} e^{-x t} \beta(t) d t
\end{aligned}
$$

and

$$
\beta(t)=o\left(t^{n}\right)
$$$$
(t \rightarrow 0)
$$ 
This follows since $\omega(u)$ is continuous at $u=0$. The total variation of $\omega(u)$ in the interval. $(0, t)$, which we denote by $V(t)$, approaches zero with $t$ and

$$
|\beta(t)|<t^{n} V(t) \text {. }
$$

If $\epsilon$ is an arbitrary positive number we can determine a number $\delta$ such that

Hence

$$
|\beta(t)|<\frac{\epsilon}{2} \frac{t^{n}}{n !} \quad(0 \leqq t \leqq \delta) .
$$

(3.12) $\left|x \int_{0}^{\delta} e^{-x t} \beta(t) d t\right|<\frac{\epsilon}{2} \frac{x}{n !} \int_{0}^{\delta} e^{-x t} t^{n} d t<\frac{\epsilon}{2} \frac{x}{n !} \int_{0}^{\infty} e^{-x t t^{n}} d t=\frac{\epsilon}{2 x^{n}}$.

By integrating (3.11) by parts one sees at once that $\beta(t)$ satisfies an inequality

$$
|\beta(t)|<M^{\prime} e^{\rho^{\prime} t} \quad(0 \leqq t<\infty),
$$

where $M^{\prime}$ and $g^{\prime}$ are suitable positive constants. Consequently

$$
\begin{aligned}
\left|x \int_{\delta}^{\infty} e^{-x t} \beta(t) d t\right|<M^{\prime} x \int_{\delta}^{\infty} e^{-t\left(x-\sigma^{\prime}\right)} d t=M^{\prime} x e^{-\delta\left(x-\sigma^{\prime}\right)} /\left(x-g^{\prime}\right) & \\
=o\left(x^{-n}\right) & (x \rightarrow \infty) .
\end{aligned}
$$

Combining inequalities (3.12) and (3.13) we have

$$
f^{(n)}(x)=o\left(x^{-n}\right) \quad(x \rightarrow \infty, n>0) .
$$

For $n=0$

In this case

$$
f(x)-\alpha(0+)=\int_{0}^{\infty} e^{-x t} d \omega(t)
$$

$$
\beta(t)=\omega(t)=o(1)
$$

and the above proof shows that

$$
f(x)-\alpha(0+)=o(1) \quad(x \rightarrow \infty),
$$

whence

$$
f(\infty)=\alpha(0+) .
$$

To show that the improper integral

$$
\int_{k / t}^{\infty} \frac{u^{k}}{k !} f^{(k+1)}(u) d u \quad\left(\frac{k}{t}>c\right)
$$

converges, we proceed by induction. The integral 


$$
\int_{k / t}^{\infty} f^{\prime}(u) d u=\lim _{R \rightarrow \infty} f(R)-f(k / t)
$$

clearly converges to the value $\alpha(0+)-f(k / t)$. Suppose that we have shown that the integral (3.15) converges for $k=m-1$. The equation

$$
\int_{k / t}^{R} f^{(m+1)}(u) \frac{u^{m}}{m !} d u=\frac{f^{(m)}(R) R^{m}}{m !}-\frac{f^{(m)}(k / t)}{m !}\left(\frac{k}{t}\right)^{m}-\int_{k / t}^{R} f^{(m)}(u) \frac{u^{m-1}}{(m-1) !} d u
$$

together with the relation (3.14) shows that (3.15) also converges for $k=m$, and thus we see that $S_{k, t}[f(x)]$ exists for every positive $t$.

Successive integration by parts shows that

$$
\begin{aligned}
S_{k, t}[f(x)]=f\left(\frac{k}{t}\right)- & f^{\prime}\left(\frac{k}{t}\right) \frac{k}{t} \\
& +\frac{1}{2 !} f^{\prime \prime}\left(\frac{k}{t}\right)\left(\frac{k}{t}\right)^{2}-\cdots+(-1)^{k} \frac{1}{k !} f^{(k)}\left(\frac{k}{t}\right)\left(\frac{k}{t}\right)^{k} .
\end{aligned}
$$

On the other hand we have

$$
\begin{aligned}
F^{(k)}(x) & =\frac{d^{k}}{d x^{k}}\left[f(x) \frac{1}{x}\right]=\sum_{n=0}^{k}(-1)^{n}\left(\begin{array}{c}
k \\
n
\end{array}\right) f^{(k-n)}(x) \frac{n !}{x^{n+1}} \\
L_{k, t}[F(x)] & =\frac{1}{k !}\left(\frac{k}{t}\right)^{k+1} \sum_{n=0}^{k}(-1)^{k-n}\left(\begin{array}{l}
k \\
n
\end{array}\right) f^{(k-n)}\left(\frac{k}{t}\right) n !\left(\frac{t}{k}\right)^{n+1}=S_{k, t}[f(x)] .
\end{aligned}
$$

Hence

$$
L_{t}[F(x)]=S_{t}[f(x)] .
$$

This completes the proof of the theorem. In the course of the proof we have established a result of interest in itself and which we state as

THEOREM 3. If the function $\phi(t)$ is of bounded variation in the interval $(0, R)$ for every finite $R$, if for some constant $c$

$$
\phi(t)=O\left(e^{c t}\right) \quad(t \rightarrow \infty),
$$

and if

$$
F(x)=\int_{0}^{\infty} e^{-x t} \phi(t) d t
$$

then

$$
L_{i}[F(x)]=\frac{\phi(t+)+\phi(t-)}{2} .
$$

Later we shall prove a much more general result of this same character. 
It is of interest to illustrate the foregoing theory by the following examples:

$$
\begin{array}{rlrl}
F(x) & =\Gamma(\gamma+1) x^{-\gamma-1}, & \phi(t)=t^{\gamma} & (\gamma \geqq 1) ; \\
f(x)=\Gamma(\gamma+1) x^{-\gamma-1}, & \alpha(t)=t^{\gamma+1} /(\gamma+1) & (0<\gamma<1) ; \\
f(x)=(x+c)^{-1}, & \alpha(t)=\left(1-e^{-c t}\right) / c & (c>0) ;
\end{array}
$$

where the limits involved in the definition of the operators $L$ and $S$ may be directly computed. Theorem 3 is not applicable to the function $\Gamma(\gamma+1) x^{-\gamma-1}$ for $\gamma<1$ since the function $\phi(t)=t r$ is not of bounded variation in any interval including the origin.

4. The inversion of the Laplace-Lebesgue integral. In this section we develop an inversion formula for the case in which $\alpha(t)$ in (1.1) is the integral of a function $\phi(t)$,

$$
\alpha(t)=\int_{0}^{t} \phi(u) d u
$$

where $\phi(u)$ is merely integrable in the sense of Lebesgue. Then (1.1) takes the form (1.2). We shall be able to show that if (1.2) converges for some value of $x$ (and hence for every greater value), then

$$
L_{t}[f(x)]=\phi(t)
$$

for almost all positive values of $t$. It is important to note that no restriction of the type (3.16) is imposed on $\phi(t)$, so that the result is the best possible one for integrals of the type (1.2). We state our result in

THEOREM 4. If the function $\phi(t)$ is integrable in the interval $(0, R)$ for every positive $R$ and if the integral

$$
f(x)=\int_{0}^{\infty} e^{-x t} \phi(t) d t
$$

converges for $x>c$, then

$$
L_{t}[f(x)]=\phi(t)
$$

for almost all positive values of $t$.

Since $\phi(t)$ is integrable we have*

$$
\int_{t_{0}}^{t}\left|\phi(u)-\phi\left(t_{0}\right)\right| d u=o\left(\left|t-t_{0}\right|\right) \quad\left(t \rightarrow t_{0}\right)
$$

for almost all positive values of $t_{0}$.

* See, for example, L. Tonelli, Serie Trigonometriche, D. 174. 
Let us fix attention on such a value $t_{0}$. Set

$$
\beta\left(t, t_{0}\right)=\int_{t_{0}}^{t}\left[\phi(u)-\phi\left(t_{0}\right)\right] d u .
$$

We wish to show that

$$
L_{t_{0}}[f(x)]=\phi\left(t_{0}\right)
$$

In view of

$$
L_{k, t_{0}}\left[\frac{\phi\left(t_{0}\right)}{x}\right]=L_{t_{0}}\left[\frac{\phi\left(t_{0}\right)}{x}\right]=\phi\left(t_{0}\right)
$$

we have only to show that the integral

$$
L_{k, t_{0}}\left[f(x)-\frac{\phi\left(t_{0}\right)}{x}\right]=\int_{0}^{\infty} e^{-k u / t_{0}}\left(\frac{k}{t_{0}}\right)^{k+1} \frac{u^{k}}{k !}\left[\phi(u)-\phi\left(t_{0}\right)\right] d u
$$

approaches zero as $k$ becomes infinite. By introducing the function $\beta\left(t, t_{0}\right)$ this becomes

$$
L_{k, t_{0}}\left[f(x)-\frac{\phi\left(t_{0}\right)}{x}\right]=\int_{0}^{\infty} e^{-k u / t_{0}}\left(\frac{k}{t_{0}}\right)^{k+1} \frac{u^{k}}{k !} d \beta\left(u, t_{0}\right) .
$$

Set

$$
\gamma(t)=\int_{0}^{t} \phi(u) d u
$$

Since the integral (4.1) converges for $x>c$, there exist positive constants $M$ and $\boldsymbol{\gamma}$ such that

$$
|\gamma(t)|<M e^{\gamma t} \quad(0 \leqq t<\infty) .
$$

On account of the relation

$$
\beta\left(t, t_{0}\right)=\gamma(t)-\gamma\left(t_{0}\right)-\phi\left(t_{0}\right)\left(t-t_{0}\right)
$$

it is clear that $\beta\left(t, t_{0}\right)$ also satisfies an inequality of the type (4.4). Hence, on integrating (4.3) by parts, we see that the integrated term vanishes if $k$ is sufficiently large $(k>g$, say). Thus

where

$$
L_{k, t_{0}}\left[f(x)-\frac{\phi\left(t_{0}\right)}{x}\right]=I(k)
$$

$$
I(k)=\left(\frac{k}{t_{0}}\right)^{k+1} \frac{1}{k !} \int_{0}^{\infty} e^{-k u / t_{0}} \beta\left(u, t_{0}\right)\left[\frac{k u^{k}}{t_{0}}-k u^{k-1}\right] d u .
$$


In the integral $I(k)$ make the change of variable $u=t_{0} y$. We thus obtain

$$
I(k)=\frac{1}{t_{0}} \frac{k^{k+1}}{(k-1) !} \int_{0}^{\infty} e^{-k} \gamma \beta\left(t_{0} y, t_{0}\right) y^{k-1}(y-1) d y .
$$

Corresponding to an arbitrary positive $\epsilon$ there is a number $\eta$ so small that

$$
\left|\beta\left(y t_{0}, t_{0}\right)\right|<\epsilon|y-1| / 3 \quad(|y-1|<\eta)
$$

by virtue of (4.2). Divide the interval of integration into the three parts $(0,1-\eta),(1-\eta, 1+\eta),(1+\eta, \infty)$, denoting the corresponding contributions by $I_{1}(k), I_{2}(k), I_{3}(k)$ respectively. Then

$$
\left|I_{1}(k)\right| \leqq \frac{1}{t_{0}} \frac{k^{k+1}}{(k-1) !} e^{-k(1-\eta)}(1-\eta)^{k-1} \int_{0}^{1}\left|\beta\left(t_{0} y, t_{0}\right)\right|(1-y) d y .
$$

The right-hand side of this inequality, and hence also the left, approaches zero with $1 / k$.

Next consider the integral $I_{3}(k)$. It converges absolutely for numbers $k$ greater than $g$. Let $k_{1}$ be such a number. Then the integral

$$
A=\int_{1}^{\infty} e^{-k_{1} y}\left|\beta\left(t_{0} y, t_{0}\right)\right| y^{k_{1}-1}(y-1) d y
$$

converges and

$$
\left|I_{3}(k)\right| \leqq \frac{1}{l_{0}} \frac{k^{k+1}}{(k-1) !} e^{-\left(k-k_{1}\right)(1+\eta)}(1+\eta)^{k-k_{1} A} .
$$

The constant $A$ is independent of $k$ and one sees easily that the right-hand side of (4.7) approaches zero with $1 / k$. Hence we may determine $k_{0}$ so large that for $k>k_{0}$

$$
\left|I_{1}(k)\right|<\epsilon / 3,\left|I_{3}(k)\right|<\epsilon / 3 .
$$

As for $I_{2}(k)$, we have by virtue of $(4.5)$

$$
\begin{aligned}
\left|I_{2}(k)\right| & <\frac{\epsilon}{3} \frac{1}{t_{0}} \frac{k^{k+1}}{(k-1) !} \int_{1-\eta}^{1+\eta} e^{-k \nu}(y-1)^{2} y^{k-1} d y \\
& <\frac{\epsilon}{3} \frac{1}{t_{0}} \frac{k^{k+1}}{(k-1) !} \int_{0}^{\infty} e^{-k \nu}(y-1)^{2} y^{k-1} d y .
\end{aligned}
$$

By use of the gamma function we see that the right-hand side of the latter inequality reduces to $\epsilon / 3$ so that

Hence

$$
|I(k)|<\epsilon \quad\left(k>k_{0}\right) .
$$




$$
L_{t_{0}}[f(x)]=\phi\left(t_{0}\right)
$$

and the proof is complete. We add the following

CoROLlaRy. If the function $\phi(t)$ is integrable in the interval $(0, R)$ for every positive $R$, is continuous at $t=t_{0}$, and if the integral

$$
\int_{0}^{\infty} e^{-x t} \phi(t) d t
$$

converges for $x>c$, then

$$
L_{t_{0}}[f(x)]=\phi\left(t_{0}\right) \quad\left(t_{0}>0\right) .
$$

By use of this result we are now able to generalize Theorem 3, removing the condition (3.16). Without loss of generality we may suppose that

$$
\phi(t)=\frac{\phi(t+)+\phi(t-)}{2} .
$$

From the function $g(t)$ of $\S 2$ we construct the function

$$
\omega(u)=\left[\phi\left(t_{0}+\right)-\phi\left(t_{0}-\right)\right] g\left(u / t_{0}\right)+\phi\left(t_{0}-\right) .
$$

This function is seen to satisfy the conditions

$$
\begin{gathered}
\omega\left(t_{0}+\right)=\phi\left(t_{0}+\right), \\
\omega\left(t_{0}-\right)=\phi\left(t_{0}-\right), \\
\omega\left(t_{0}\right)=\phi\left(t_{0}\right) .
\end{gathered}
$$

Consequently the function $\phi(u)-\omega(u)$ has the value zero at $u=t_{0}$ and is continuous there. Set

$$
G(x)=\int_{0}^{\infty} e^{-x u} \omega(u) d u .
$$

The corollary of Theorem 4 gives us

$$
\begin{aligned}
L_{t_{\mathrm{o}}}[F(x)-G(x)] & =\phi\left(t_{0}\right)-\omega\left(t_{0}\right)=0, \\
L_{t_{0}}[F(x)] & =L_{t_{0}}[G(x)] .
\end{aligned}
$$

Since $\omega(u)$ is bounded, and thus satisfies condition (3.16), it follows that we may apply Theorem 3 to $G(x)$. Thus

$$
L_{t_{0}}[G(x)]=\omega\left(t_{0}\right)=\phi\left(t_{0}\right)
$$

and

$$
L_{t_{0}}[F(x)]=\phi\left(t_{0}\right)
$$


We have thus proved

THEOREM 5. If the function $\phi(t)$ is of bounded variation in the interval $(0, R)$ for every positive $R$, and if

$$
F(x)=\int_{0}^{\infty} e^{-x t} \phi(t) d t
$$

the integral converging for some value of $x$, then

$$
L_{t}[F(x)]=\frac{\phi(t+)+\phi(t-)}{2} .
$$

5. Uniform convergence. We have seen that if $\phi(t)$ is continuous at $t=t_{0}$ then $L_{t_{0}}[f(x)]=\phi\left(t_{0}\right)$. If $\phi(t)$ is continuous in an interval $a \leqq t \leqq b$, then the equation holds for each $t$ of the interval. We now show further that as $k$ becomes infinite the sequence of functions $L_{k, t}[f(x)]$ tends to $\phi(t)$ uniformly in any closed sub-interval of $(a, b)$ not including the end points. The precise result is stated in

Theorem 6. If the function $\phi(t)$-is integrable in the interval $(0, R)$ for every positive $R$, is continuous in the interval $0 \leqq a \leqq t \leqq b$, and if the integral

$$
f(x)=\int_{0}^{\infty} e^{-x t} \phi(t) d t
$$

converges for some value of $x$, then

$$
\lim _{k \rightarrow \infty} L_{k, t}[f(x)]=\phi(t)
$$

uniformly in the interval $a^{\prime} \leqq t \leqq b^{\prime}$, where $a<a^{\prime}<b^{\prime}<b$.

Defining the function $\beta\left(t, t_{0}\right)$ as in the proof of Theorem 4 , we obtain

$$
L_{k, t}[f(x)]-\phi(t)=\int_{0}^{\infty} e^{-k u / t}\left(\frac{k}{t}\right)^{k+1} \frac{u^{k}}{k !} d \beta(u, t) .
$$

By virtue of (4.4) we have

$$
|\beta(u, t)| \leqq M e^{\gamma u}+M e^{\gamma t}+|\phi(t)||u-t| .
$$

Denote by $N$ the maximum of $\phi(t)$ in the interval $a \leqq t \leqq b$. Then

$$
|\beta(u, t)| \leqq M e^{\gamma u}+M e^{\gamma b}+N(u+b) \leqq M^{\prime} e^{\gamma u} \quad(0 \leqq u<\infty)
$$

if $M^{\prime}$ is suitably chosen. Hence, on integrating (5.2) by parts the integrated 
term vanishes for all $t$ in the interval $(a, b)$ provided $k>b \gamma$. Then

$$
I(k)=L_{k, t}[f(x)]-\phi(t)=\frac{k^{k+1}}{(k-1) !} \frac{1}{t^{k+2}} \int_{0}^{\infty} e^{-k u / t} \beta(u, t) u^{k-1}(u-t) d u .
$$

Make the change of variable $v=u / t$ :

$$
I(k)=\frac{k^{k+1}}{(k-1) !} \frac{1}{t} \int_{0}^{\infty} e^{-k v} \beta(t v, t) v^{k-1}(v-1) d v .
$$

Since $\phi(t)$ is continuous in the closed interval $(a, b)$, to an arbitrary positive $\epsilon$ there corresponds a number $\delta$ such that for any two points $t^{\prime}$ and $t^{\prime \prime}$ of $(a, b)$

$$
\left|\phi\left(t^{\prime}\right)-\phi\left(t^{\prime \prime}\right)\right|<\epsilon / 3
$$

provided only that $\left|t^{\prime}-t^{\prime \prime}\right|<\delta$. Now choose a number $\eta$ satisfying the inequalities

$$
0<\eta<1, \eta \leqq \delta / b^{\prime}, \eta \leqq\left(b-b^{\prime}\right) / b^{\prime}, \eta \leqq\left(a^{\prime}-a\right) / a^{\prime} .
$$

With this number $\eta$ define the integrals $I_{1}(k), I_{2}(k), I_{3}(k)$ as in $\$ 4$. From (4.6) we now obtain

$$
\left|I_{1}(k)\right| \leqq \frac{1}{a^{\prime}} \frac{k^{k+1}}{(k-1) !} e^{-k(1-\eta)}(1-\eta)^{k-1} M^{\prime} \int_{0}^{1} e^{\gamma b \nu}(1-y) d y .
$$

The right-hand side of this inequality is independent of $t$ and tends to zero with $1 / k$.

We consider next the integral $I_{3}(k)$. Determine $k_{1}>\gamma b$. Then from (4.7) we obtain

where

$$
\left|I_{3}(k)\right| \leqq \frac{1}{a^{\prime}} \frac{k^{k+1}}{(k-1) !} e^{-\left(k-k_{1}\right)(1+\eta)}(1+\eta)^{k-k_{1}} B
$$

$$
B=\int_{1}^{\infty} e^{-k_{1} y} e^{\gamma b y} y^{k_{1}-1}(y-1) d y .
$$

Again the right-hand side is independent of $t$ and approaches zero with $1 / k$.

In order to discuss $I_{2}(k)$ we note first that

$$
|\beta(t v, t)| \leqq \epsilon t|v-1| / 3
$$

if $1-\eta \leqq v \leqq 1+\eta$ and if $a^{\prime} \leqq t \leqq b^{\prime}$. For, these inequalities imply that

$$
|t v-t|=t|v-1| \leqq b^{\prime} \eta \leqq \delta .
$$

Moreover $t v$ and $t$ lie in the closed interval $(a, b)$ since the inequalities 


$$
0 \leqq t \leqq b^{\prime}, 0 \leqq v \leqq 1+\eta
$$

imply

$$
t v \leqq(1+\eta) b^{\prime},
$$

or, by virtue of (5.4),

$$
t v \leqq b .
$$

In a similar way the inequalities $t \geqq a^{\prime}$ and $v \geqq 1-\eta$ imply $t v \geqq a$. It follows that

Hence

$$
|\dot{\beta}(t v, t)| \leqq\left|\int_{t}^{t v}\right| \phi(u)-\phi(t)|d u| \leqq t|v-1| \epsilon / 3 .
$$

$$
\begin{aligned}
\left|I_{2}(k)\right| & \leqq \frac{k^{k+1}}{(k-1) !} \frac{1}{t} \frac{\epsilon}{3} \int_{1-\eta}^{1+\eta} e^{-k v} v^{k-1}|v-1|^{2} t d v \\
& <\frac{k^{k+1}}{(k-1) !} \frac{\epsilon}{3} \int_{0}^{\infty} e^{-k v} v^{k-1}(v-1)^{2} d v=\frac{\epsilon}{3} .
\end{aligned}
$$

Consequently we may determine a number $k_{2}$ independent of $t$ such that for $k>k_{2}$

$$
|I(k)|<\epsilon,
$$

and the proof is complete.

From this result follows immediately

THEOREM 7. If the function $\alpha(t)$ is of bounded variation in the interval $(0, R)$ for every positive $R$, is continuous in the interval $0 \leqq a \leqq t \leqq b$, if $\alpha(0)=0$, and if the integral

$$
f(x)=\int_{0}^{\infty} e^{-x t} d \alpha(t)
$$

converges for some $x$, then

$$
\lim _{k \rightarrow \infty} S_{k, t}[f(x)]=\alpha(t)
$$

uniformly in the interval $a^{\prime} \leqq t \leqq b^{\prime}$, where $a<a^{\prime}<b^{\prime}<b$.

For if $x$ is sufficiently large

$$
f(x) / x=\int_{0}^{\infty} e^{-x t} \alpha(t) d t,
$$

and we have already seen that

$$
L_{k, t}[f(x) / x]=S_{k, t}[f(x)] .
$$


By Theorem 6 the left-hand member of this equation, and hence also the right, approaches $\alpha(t)$ uniformly in the interval $\left(a^{\prime}, b^{\prime}\right)$.

The interval of uniform convergence may, under certain conditions, be extended to infinity. For example, we have

THEOREM 8. If the function $\phi(t)$ is continuous in the interval $0 \leqq t<\infty$, and if $\phi(t)$ tends to a finite limit as $t$ becomes infinite, then

$$
\lim _{k \rightarrow \infty} L_{k, t}[f(x)]=\phi(t)
$$

uniformly in that interval provided $L_{k, 0}[f(x)]$ is defined as $\phi(0)$.

Under the present hypotheses the integral (5.1) converges absolutely for $x>0$ and the method of proof is greatly simplified on that account. It will be seen that we must show that

$$
\lim _{k \rightarrow \infty} \frac{k^{k+1}}{k !} \int_{0}^{\infty} e^{-k y} y^{k}[\phi(t y)-\phi(t)] d y
$$

approaches zero uniformly in the interval $0 \leqq t<\infty$. The details of the proof are left to the reader.

This theorem is a result which the author stated without proof in an earlier note.* Another result which we enunciated in that note we record here as a

COROLlary. The function

$$
(1+x)^{-k}-e^{-k x}
$$

tends uniformly to zero in the interval $0 \leqq x<\infty$ as $k$ becomes infinite.

To prove this take the function $f(x)$ of Theorem 8 as $(1+x)^{-1}$ and $\phi(t)=e^{-t}$. Then

$$
\frac{1}{1+x}=\int_{0}^{\infty} e^{-x t} e^{-t} d t \quad(x>-1) .
$$

Since $e^{-t}$ approaches zero as $t$ becomes infinite and is continuous in the interval $0 \leqq t<\infty$, Theorem 8 is applicable, so that

$$
\lim _{k \rightarrow \infty} L_{k, t}\left[\frac{1}{x+1}\right]=\lim _{k \rightarrow \infty}\left(1+\frac{t}{k}\right)^{-k-1}=e^{-t}
$$

* This was the Proceedings article mentioned in the Introduction. See Theorems 1 and 3 of that note. It will be found that the statement of Theorem 3 is somewhat different from the statement of Theorem 8 above, but the equivalence of the two results may be seen by making the change of variable $k / x=t$. 
uniformly for $0 \leqq t<\infty$. If we set $t=k x$ we have

$$
\lim _{k \rightarrow \infty}\left[(1+x)^{-k-1}-e^{-k x}\right]=0
$$

uniformly for $0 \leqq x<\infty$. But

$$
(1+x)^{-k}-e^{-k x}=\left[(1+x)^{-k-1}-e^{-k x}\right]+x(1+x)^{-k-1} .
$$

The minimum of the function $x(1+x)^{-k-1}$ in the interval $0 \leqq x<\infty$ is $k^{k}(k+1)^{-k-1}$. This approaches zero with $1 / k$, so that the corollary is proved.

6. Further inversion formulas. We now prove

THEOREM 9. If the integral

$$
f(x)=\int_{0}^{\infty} e^{-x t} d \alpha(t) \quad(\alpha(0)=0)
$$

converges for some value of $x$, then

$$
\begin{gathered}
\lim _{k \rightarrow \infty}\left[f(\infty)+(-1)^{k+1} \int_{k / t}^{\infty} \frac{u^{k}}{k !} e^{c k / u} f^{(k+1)}(u+c) d u\right] \\
=\frac{\alpha(t+)+\alpha(t-)}{2}
\end{gathered}
$$

for any constant c.

This theorem is a generalization of Theorem 2 and reduces to that result when $c$ is put equal to zero. To prove it we note as before that for sufficiently large positive values of $x$ we have

Since

$$
F(x)=f(x) / x=\int_{0}^{\infty} e^{-x t} \alpha(t) d t .
$$

$$
F(x+c)=\int_{0}^{\infty} e^{-x t} e^{-c t} \alpha(t) d t
$$

we have by an application of Theorem 3

$$
\lim _{k \rightarrow \infty} \frac{(-1)^{k} e^{c t}}{k !} F^{(k)}\left(\frac{k}{t}+c\right)\left(\frac{k}{t}\right)^{k+1}=\frac{\alpha(t+)+\alpha(t-)}{2} .
$$

Set

$$
I_{k}=f(\infty)+(-1)^{k+1} \int_{k / t}^{\infty} \frac{u^{k}}{k !} e^{c k / u} f^{(k+1)}(u+c) d u .
$$

That this integral converges for any $c$ and for sufficiently large values of $k$ will become apparent when we replace $f(x)$ by $x F(x)$ : 
$I_{k}=f(\infty)+(-1)^{k+1} \int_{k / t}^{\infty} \frac{u^{k}}{k !} e^{c k / u}\left[F^{(k+1)}(u+c)(u+c)+(k+1) F^{(k)}(u+c)\right] d u$.

By integration by parts we obtain

$$
\begin{aligned}
I_{k}=f(\infty)+ & \frac{(-1)^{k}}{k !}\left(\frac{k}{t}+c\right)\left(\frac{k}{t}\right)^{k} e^{c t} F^{(k)}\left(\frac{k}{t}+c\right) \\
+ & \lim _{u \rightarrow \infty}(-1)^{k+1}(u+c) \frac{u^{k}}{k !} e^{c k / u} F^{(k)}(u+c) \\
& +(-1)^{k+1} \int_{k / t}^{\infty} \frac{e^{c k / u}}{k !} c^{2} k u^{k-2} F^{(k)}(u+c) d u .
\end{aligned}
$$

To show that the integral (6.1) exists it will be sufficient to show that the limit involved in the third term of (6.2) exists and that the integral of (6.2) converges. But we saw in $\$ 3$ that

$$
(-1)^{k} F^{(k)}(x) x^{k+1} / k !=f(x)-f^{\prime}(x) x+f^{\prime \prime}(x) \frac{x^{2}}{2 !}-\cdots+(-1)^{k} f^{(k)}(x) \frac{x^{k}}{k !}
$$

and that

$$
\lim _{x \rightarrow \infty} f^{(i)}(x) x^{i}=0 \quad(i=1,2, \cdots) .
$$

Hence

$$
\lim _{x \rightarrow \infty}(-1)^{k} F^{(k)}(x) x^{k+1} / k !=f(\infty) \quad(k=0,1,2, \cdots),
$$

or

$$
\lim _{u \rightarrow \infty}(-1)^{k+1} \frac{u^{k}}{k !}(u+c) e^{c k / u} F^{(k)}(u+c)=-f(\infty),
$$

so that the first and third terms of (6.2) may be omitted. To show that the integral (6.2) converges we appeal to the inequality (3.4), from which it foliows immediately that

$$
\left|F^{(k)}(x)\right|<M k ! /(x-g)^{k+1} \quad(x>g) .
$$

By application of this inequality we see easily that the integral in question is $O(1 / k)$ as $k$ becomes infinite. Since the second term of (6.2) approaches $[\alpha(t+)+\alpha(t-)] / 2$, we see that

$$
\lim _{k \rightarrow \infty} I_{k}=\frac{\alpha(t+)+\alpha(t-)}{2} .
$$

This completes the proof of the theorem. 
7. Relation between the operators $L$ and $S$. We have already seen that if

$$
f(x)=\int_{0}^{\infty} e^{-x t} \phi(t) d t
$$

then

$$
S_{t}[f(x)]=\int_{0}^{t} \phi(u) d u, \quad L_{t}[f(x)]=\phi(t) .
$$

That is, we are able to determine $\phi(t)$ and its integral in terms of $f(x)$. We are thus led to seek to determine the successive integrals and the successive derivatives (provided the latter exist) of $\phi(t)$ in terms of $f(x)$. It will appear in this section that the successive integrals of $S_{k, t}[f(x)]$ approach the corresponding integrals of $\phi(t)$ and that the successive derivatives of $L_{k, t}[f(x)]$ approach the corresponding derivatives of $\phi(t)$. We begin by proving

THEOREM 10. If $f(x)$ is any function such that $S_{k, t}[f(x)]$ exists for every positive $t$, then

$$
\frac{d}{d t} S_{k, t}[f(x)]=-\frac{1}{t} L_{k, t}\left[f^{\prime}(x)\right]
$$

almost everywhere in the interval $(0, \infty)$.

By hypothesis $f(x)$ must have derivatives of the first $k+1$ orders and the integral

$$
(-1)^{k+1} \int_{k / t}^{\infty} \frac{u^{k}}{k !} f^{(k+1)}(u) d u
$$

must converge for $t>0$. But this integral has a derivative with respect to $t$ almost everywhere in the interval $(0, \infty)$ equal to

$$
\frac{(-1)^{k+1}}{k ! t}\left(\frac{k}{t}\right)^{k+1} f^{(k+1)}\left(\frac{k}{t}\right)
$$

so that the result is proved.

If $f(x)$ is defined by (7.1) where $\phi(t)$ is integrable in $(0, R)$ for every positive $R$, Theorem 2 shows that

$$
\lim _{k \rightarrow \infty} S_{k, t}[f(x)]=\int_{0}^{t} \phi(u) d u .
$$

Theorem 10 shows further that the first derivative of $S_{k, t}[f(x)]$ with respect to $t$ approaches $\phi(t)$ almost everywhere. For

$$
f^{\prime}(x)=-\int_{0}^{\infty} e^{-x t} t \phi(t) d t
$$


and by Theorem 4

$$
\lim _{k \rightarrow \infty}-\frac{1}{t} L_{k, t}\left[f^{\prime}(x)\right]=\phi(t)
$$

We next establish

THEOREM 11. If the function $\phi(t)$ is integrable in $(0, R)$ for every positive $R$, and if the integral

$$
f(x)=\int_{0}^{\infty} \dot{e}^{-x t} \phi(t) d t
$$

converges for some value of $x$, then

$$
\begin{aligned}
& \int_{0}^{t} d u_{m} \int_{0}^{u_{m}} d u_{m-1} \int_{0}^{u_{m-1}} \cdots \int_{0}^{u_{1}} \phi\left(u_{0}\right) d u_{0} \\
&=\lim _{k \rightarrow \infty} \frac{(-1)^{k+1}}{k ! m !} \int_{k / t}^{\infty} u^{k} f^{(k+1)}(u)\left(t-\frac{k}{u}\right)^{m} d u .
\end{aligned}
$$

It is a familiar fact that the iterated integral (7.2) can be expressed as the single integral

$$
\int_{0}^{t} \frac{(t-u)^{m}}{m !} \phi(u) d u
$$

The integral on the right-hand side of (7.2) can be expressed as follows:

$$
\begin{aligned}
& \frac{(-1)^{k+1}}{k ! m !} \int_{k / t}^{\infty} u^{k} f^{(k+1)}(u)\left(t-\frac{k}{u}\right)^{m} d u \\
& =\frac{(-1)^{k+1}}{k ! m !} \sum_{i=0}^{m}(-1)^{i}\left(\begin{array}{c}
m \\
i
\end{array}\right) t^{m-i} k^{i} \int_{k / t}^{\infty} u^{k-i} f^{(k+1)}(u) d u \quad(k>m) .
\end{aligned}
$$

That the integrals on the right-hand side of this equality converge one sees at once by use of (3.10) for $i=1,2, \cdots, m$. For $i=0$ the convergence of the integral was already established in $\S 3$. Now let $k$ become infinite in (7.3). We shall be able to show that

$$
\lim _{k \rightarrow \infty} \frac{(-1)^{k+1}}{k !} k^{i} \int_{k / t}^{\infty} f^{(k+1)}(u) u^{k-i} d u=\int_{0}^{t} u^{i} \phi(u) d u
$$

and thus that

$$
\begin{aligned}
& \lim _{k \rightarrow \infty} \frac{(-1)^{k+1}}{k ! m !} \sum_{i=0}^{m}(-1)^{i}\left(\begin{array}{c}
m \\
i
\end{array}\right) t^{m-i} k^{i} \int_{k / t}^{\infty} u^{k-i} f^{(k+1)}(u) d u \\
& =\sum_{i=0}^{m}(-1)^{i}\left(\begin{array}{c}
m \\
i
\end{array}\right) \frac{t^{m-i}}{m !} \int_{0}^{t} u^{i} \phi(u) d u=\frac{1}{m !} \int_{0}^{t}(t-u)^{m} \phi(u) d u .
\end{aligned}
$$


This will clearly establish the theorem. To prove (7.4) we note first that

$$
\begin{aligned}
& \int_{k / t}^{\infty} u^{k-i} f^{(k+1)}(u) d u=\int_{\left(k^{\prime}+i\right) / t}^{\infty} u^{k^{\prime}} f^{\left(k^{\prime}+i+1\right)}(u) d u \\
& =\int_{k^{\prime} / t}^{\infty} u^{k^{\prime}} f^{\left(k^{\prime}+i+1\right)}(u) d u-\int_{k^{\prime} / t}^{\left(k^{\prime}+i\right) / t} u^{k^{\prime}} f^{\left(k^{\prime}+i+1\right)}(u) d u,
\end{aligned}
$$

where $k^{\prime}=k-i$. But

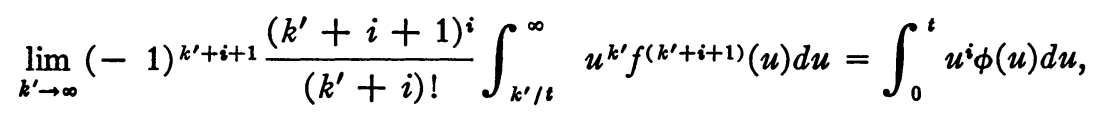

as one sees by applying Theorem 2 to the function

and by noting that

$$
f^{(i)}(x)=(-1)^{i} \int_{0}^{\infty} e^{-x t} t^{i} \phi(t) d t
$$

$$
\frac{1}{k^{\prime} !} \sim \frac{\left(k^{\prime}+i+1\right)^{i}}{\left(k^{\prime}+i\right) !}
$$

That $f^{(i)}(\infty)=0$ follows from (3.10).

It remains only to show that

$$
\lim _{k \rightarrow \infty} I_{k}=0,
$$

where

Set

$$
I_{k}=\frac{(k+i+1)^{i}}{(k+i) !} \int_{k / t}^{(k+i) / t} u^{k} f^{(k+i+1)}(u) d u=0 .
$$

Then

$$
\alpha(t)=\int_{0}^{t} \phi(y) d y .
$$

$$
F(x)=f(x) / x=\int_{0}^{\infty} e^{-x t} \alpha(t) d t,
$$

and $\alpha(t)$ satisfies the inequality (3.4). Introducing the function $F(x)$ we have

$$
I_{k}=\frac{(k+i+1)^{i}}{(k+i) !} \int_{k / t}^{(k+i) / t}\left[F^{(k+i+1)}(u) u^{k+1}+(k+i+1) u^{k} F^{(k+i)}(u)\right] d u .
$$

By integration by parts this becomes

$$
\begin{aligned}
I_{k}=\frac{(k+i+1)^{i}}{(k+i) !} & {\left[F^{(k+i)}\left(\frac{k+i}{t}\right)\left(\frac{k+i}{t}\right)^{k+1}\right.} \\
& \left.-F^{(k+i)}\left(\frac{k}{t}\right)\left(\frac{k}{t}\right)^{k+1}+i \int_{k / t}^{(k+i) / t} F^{(k+i)}(u) u^{k} d u\right] .
\end{aligned}
$$


If we apply Theorem 3 to the function

$$
(-1)^{i} F^{(i)}(x)=\int_{0}^{\infty} e^{-x t} t^{i} \alpha(t) d t
$$

and to the function $F(x)$ itself, we see that the sum of the first two terms on the right-hand side of (7.5) approaches zero with $1 / k$. Finally by virtue of (2.3) we have

$$
\left|F^{(k+i)}(u)\right|<M(k+i) ! /(u-g)^{k+i+1} \quad(u>g),
$$

whence the third term of $(7.5)$ becomes $O(1 / k)$ as $k$ becomes infinite. The proof of the theorem is thus complete.

We turn next to the problem of determining the successive derivatives of $\phi(t)$ in terms of $f(x)$. We first establish the following

LEMMA. If the function $f(x)$ is of class* $C^{n}$ in the interval $0 \leqq x<\infty$, then

$$
\begin{aligned}
L_{k, t}\left[\frac{d^{n}}{d x^{n}}\left\{x^{n} f(x)\right\}\right]=(-1)^{n} t^{n} \frac{d^{n}}{d t^{n}}\left\{L_{k, t}[f(x)]\right\} & \\
& (t>0 ; n, k=0,1,2, \cdots) .
\end{aligned}
$$

We prove this result by induction. For $n=1$ we have

$$
\begin{gathered}
\frac{d}{d x}\{x f(x)\}=x f^{\prime}(x)+f(x), \\
L_{k, t}[x f(x)]=(-1)^{k}\left[f^{(k+1)}(k / t)(k / t)^{k+2}+(k+1) f^{(k)}(k / t)(k / t)^{k+1}\right] / k ! .
\end{gathered}
$$

On the other hand

$$
\frac{d}{d t} L_{k, t}[f(x)]=\frac{(-1)^{k+1}}{k !}\left[f^{(k+1)}\left(\frac{k}{t}\right)\left(\frac{k}{t}\right)^{k+1} \frac{k}{t^{2}}+(k+1) f^{(k)}\left(\frac{k}{t}\right) \frac{k}{t^{2}}\left(\frac{k}{t}\right)^{k}\right] \text {, }
$$

so that (7.7) is established for $n=1$. Suppose it is true for $0,1, \cdots, n-1$. Rewrite the left-hand member of (7.7) as follows:

$$
L_{k, t}\left[\frac{d^{n-1}}{d x^{n-1}}\left\{x^{n-1} \frac{d}{d x} x f(x)+(n-1) x^{n-2} x f(x)\right\}\right] .
$$

If we replace $n$ by $n-1$ and $f(x)$ by

$$
(n-1) f(x)+\frac{d}{d x}[x f(x)]
$$

in (7.7) we see that (7.8) becomes

* That is, continuous with its first $n$ derivatives. 


$$
(-1)^{n-1} t^{n-1} \frac{d^{n-1}}{d t^{n-1}} L_{k, t}\left[\frac{d}{d x}\{x f(x)\}+(n-1) f(x)\right] .
$$

Applying (7.7) again, now with $n=1,(7.8)$ becomes

$$
\begin{gathered}
(-1)^{n-1} t^{n-1} \frac{d^{n-1}}{d t^{n-1}}\left\{(n-1) L_{k, t}[f(x)]-t \frac{d}{d t} L_{k, t}[f(x)]\right\} \\
=(-1)^{n} t^{n} \frac{d^{n}}{d t^{n}} L_{k, t}[f(x)] .
\end{gathered}
$$

The induction is thus complete.

By use of this Lemma we establish

THEOREM 12. If the function $\phi(t)$ is of class $C^{n}$ in the interval $0 \leqq t<\infty$, and if the integral

$$
\int_{0}^{\infty} e^{-\dot{x} t} \phi^{(n)}(t) d t
$$

converges for some value of $x$, then the integral

$$
f(x)=\int_{0}^{\infty} e^{-x t} \phi(t) d t
$$

also converges for large values of $x$ and

$$
\phi^{(n)}(t)=\lim _{k \rightarrow \infty} \frac{d^{n}}{d t^{n}} L_{k, t}[f(x)]
$$

Since the integral (7.9) converges, an application of inequality (3.4) gives (7.11)

$$
\left|\phi^{(n-1)}(t)\right|<M e^{o t}
$$$$
(0 \leqq t<\infty) \text {. }
$$

Integrating (7.9) by parts and using (7.11) we obtain

$$
\int_{0}^{\infty} e^{-x t} \phi^{(n)}(t) d t=-\phi^{(n-1)}(0)+x \int_{0}^{\infty} e^{-x t} \phi^{(n-1)}(t) d t
$$

for all values of $x$ sufficiently large. Successive applications of this result will show that (7.10) converges and that

$$
f(x) x^{n}=\phi^{(n-1)}(0)+x \phi^{(n-2)}(0)+\cdots+x^{n-1} \phi(0)+\int_{0}^{\infty} e^{-x t} \phi^{(n)}(t) d t .
$$

Differentiate both sides of this equation $n$ times with respect to $x$,

$$
\frac{d^{n}}{d x^{n}}\left(x^{n} f(x)\right)=(-1)^{n} \int_{0}^{\infty} e^{-x t} t^{n} \phi^{(n)}(t) d t,
$$


and apply the operator $L_{k, t}$ to both sides of the resulting equation. This gives, when the result of the Lemma is taken into account,

$$
t^{n} \frac{d^{n}}{d t^{n}} L_{k, t}[f(x)]=L_{k, t}\left[\int_{0}^{\infty} e^{\left.-x t t^{n} \phi^{(n)}(t) d t\right] .}\right.
$$

Take the limit of both sides of this equation as $k$ becomes infinite, applying Theorem 4, and obtain

$$
t^{n} \phi^{(n)}(t)=\lim _{k \rightarrow \infty} t^{n} \frac{d^{n}}{d t^{n}} L_{k, t}[f(x)]
$$

from which the result of the theorem follows immediately.

8. The operator $L$ applied to the Laplace-Stieltjes integral. We conclude Part I with a discussion of the effect of the operator $L$ on functions $f(x)$ which are defined by Laplace-Stieltjes integrals (1.1). We have already seen that

$$
S_{t}[f(x)]=\frac{\alpha(t+)+\alpha(t-)}{2} .
$$

We now show that $L_{t}[f(x)]$ also exists for certain values of $t$. We prove

THEOREM 13. Let the function $\alpha(t)$ be of bounded variation in the interval $(0, R)$ for every positive $R$, and let it possess a derivative on the right $\alpha_{+}{ }^{\prime}\left(t_{0}\right)$ and $a$ derivative on the left $\alpha_{-}{ }^{\prime}\left(t_{0}\right)$ at a point $t_{0}>0$. Then if the integral

$$
f(x)=\int_{0}^{\infty} e^{-x t} d \alpha(t)
$$

converges for some value of $x$,

$$
L_{t_{0}}[f(x)]=\frac{\alpha_{+}^{\prime}\left(t_{0}\right)+\alpha_{-}^{\prime}\left(t_{0}\right)}{2} .
$$

We note first that it will be no essential restriction to suppose that $t_{0}=1$. For, set

$$
g(x)=f\left(\frac{x}{t_{0}}\right)=\int_{0}^{\infty} e^{-x t / t_{0}} d \alpha(t)=\int_{0}^{\infty} e^{-x u} d \alpha\left(t_{0} u\right)
$$

where $t=t_{0} u$. Simple computation shows that

$$
L_{1}[g(x)]=t_{0} L_{t_{0}}[f(x)]
$$

and that the derivatives on the right and left of $\alpha\left(t_{0} u\right)$ at $u=1$ are $t_{0} \alpha_{+}{ }^{\prime}\left(t_{0}\right)$ and $t_{0} \alpha_{-}^{\prime}\left(t_{0}\right)$ respectively. Hence if we have proved the theorem for $t_{0}=1$ we have 


$$
L_{t_{0}}[f(x)]=\frac{L_{1}[g(x)]}{t_{0}}=\frac{\alpha_{+}^{\prime}\left(t_{0}\right)+\alpha_{-}^{\prime}\left(t_{0}\right)}{2} .
$$

Form the expression

$$
L_{k, 1}[f(x)]=\frac{k^{k+1}}{k !} \int_{0}^{\infty} e^{-k t} t^{k} d \alpha(t) .
$$

The improper integral will converge if $k$ is sufficiently large. It will be sufficient to show that the two integrals

$$
\begin{aligned}
& I_{k}=\frac{k^{k+1}}{k !} \int_{0}^{1} e^{-k \ell t t^{k}} d\left[\alpha(t)-\alpha_{-}^{\prime}(1) t\right], \\
& \left.J_{k}=\frac{k^{k+1}}{k !} \int_{1}^{\infty} e^{-k t t^{k} d} d \alpha(t)-\alpha_{+}^{\prime}(1) t\right]
\end{aligned}
$$

approach zero with $1 / k$, since we saw in $\$ 2$ that

$$
\begin{aligned}
& \lim _{k \rightarrow \infty} \frac{k^{k+1}}{k !} \int_{0}^{1} e^{-k t} t^{k} \alpha_{-}^{\prime}(1) d t=\frac{\alpha^{\prime}(1)}{2}, \\
& \lim _{k \rightarrow \infty} \frac{k^{k+1}}{k !} \int_{1}^{\infty} e^{-k t} t^{k} \alpha_{+}^{\prime}(1) d t=\frac{\alpha_{+}^{\prime}(1)}{2} .
\end{aligned}
$$

If we introduce the functions

$$
\begin{aligned}
& \beta(t)=\alpha(1)-\alpha(t)-\alpha_{-}^{\prime}(1)(1-t), \\
& \gamma(t)=\alpha(t)-\alpha(1)-\alpha_{+}^{\prime}(1)(t-1),
\end{aligned}
$$

we have

$$
\begin{aligned}
& I_{k}=\frac{-k^{k+1}}{k !} \int_{0}^{1} e^{-k t} t k d \beta(t), \\
& J_{k}=\frac{k^{k+1}}{k !} \int_{1}^{\infty} e^{-k t} t^{k} d \gamma(t)
\end{aligned}
$$

By noting that

$$
\begin{array}{ll}
\beta(t)=o(1-t) & (t \rightarrow 1-), \\
\gamma(t)=o(t-1) & (t \rightarrow 1+),
\end{array}
$$

and by use of the methods of $\$ 4$, we see that $I_{k}$ and $J_{k}$ approach zero with $1 / k$. We omit the details.

The proof may easily be extended to include the case in which $\alpha(t)$ has right-hand or left-hand derivatives that are infinite at $t_{0}$. 


\section{PART II}

The REPRESENTATION OF FUNCTIONS AS LAPLACE INTEgRaLS

9. A preliminary formula. In Part I we dealt with functions $f(x)$ which were known to be expressible as Laplace integrals. Here we shall abandon this assumption and assume only that the functions $f(x)$ are such as to make the operators $L$ and $S$ have meaning. This will lead us to certain uniqueness theorems regarding the representation of functions as Laplace integrals and to necessary and sufficient conditions for such representation. We begin with

THeOREM 14. If $f(x)$ is of class $C^{n}$ in the interval $c \leqq x<\infty$, and if the integrals

$$
\int_{c}^{\infty} u^{k} f^{(k+1)}(u) d u \quad(k=0,1,2, \cdots, n-1)
$$

converge, then

$$
\frac{(-1)^{k}}{k !} F^{(k)}(x) x^{k+1}=f(\infty)+\frac{1}{k !} \int_{x}^{\infty} u^{k}(-1)^{k+1} f^{(k+1)}(u) d u
$$

where $F(x)=f(x) / x$.

$$
(x \geqq c ; k=0,1, \cdots, n-1)
$$

By integration by parts we obtain

$$
\begin{aligned}
(-1)^{k+1} \int_{x}^{\infty} \frac{u^{k}}{k !} f^{(k+1)}(u) d u= & {\left[(-1)^{k+1} f^{(k)}(u) \frac{u^{k}}{k !}\right]_{x}^{\infty} } \\
& +(-1)^{k} \int_{x}^{\infty} \frac{u^{k-1}}{(k-1) !} f^{(k)}(u) d u .
\end{aligned}
$$

Since both integrals converge by hypothesis it follows that the limits

$$
\lim _{x \rightarrow \infty} x^{k} f^{(k)}(x)
$$

exist for $k=0,1, \cdots, n-1$. The existence of all of these limits implies that they are zero except perhaps for $k=0$. We prove this by induction. Suppose that

$$
\lim _{x \rightarrow \infty} x f^{\prime}(x)=B>0 .
$$

Then there exists a positive number $x_{0}$ such that

Hence

$$
x f^{\prime}(x)>B / 2 \quad\left(x \geqq x_{0}>0\right) .
$$




$$
\begin{aligned}
\int_{x_{0}}^{x} f^{\prime}(u) d u & >\frac{B}{2} \int_{x_{0}}^{x} \frac{d u}{u}, \\
f(x) & >\frac{B}{2} \log \frac{x}{x_{0}}+f\left(x_{0}\right) .
\end{aligned}
$$

As $x$ becomes positively infinite the right-hand side of this inequality does also, so that $f(x)$ can not approach a limit as the hypothesis demands. If $B$ is negative we need only replace $f(x)$ by $-f(x)$ in the foregoing proof.

Now suppose we have proved that

$$
\lim _{x \rightarrow \infty} x^{k} f^{(k)}(x)=0 \text {. }
$$

Then

$$
x \frac{d}{d x}\left(x^{k} f^{(k)}(x)\right)=x^{k+1} f^{(k+1)}(x)+k x^{k} f^{(k)}(x)
$$

approaches a limit by hypothesis. By the previous work this limit must be zero, whence

$$
\lim _{x \rightarrow \infty} x^{k+1} f^{(k+1)}(x)=0 .
$$

This completes the induction and gives us the equation

$(-1)^{k+1} \int_{x}^{\infty} \frac{u^{k}}{k !} f^{(k+1)}(u) d u=(-1)^{k} \frac{f^{(k)}(x) x^{k}}{k !}+(-1)^{k} \int_{x}^{\infty} \frac{u^{k-1}}{(k-1) !} f^{(k)}(u) d u$.

Successive application of this result gives (9.2).

COROLLARY 1. Under the conditions of the theorem,

$$
L_{k, t}[F(x)]=S_{k, t}[f(x)] \quad(0<t \leqq k / c ; k=0,1, \cdots, n-1)
$$

if $c>0$.

CoROLlary 2. If condition (9.1) is replaced by the condition

$$
\lim _{x \rightarrow \infty} x^{k} f^{(k)}(x) \text { exists } \quad(k=0,1,2, \cdots, n-1),
$$

the result of the theorem remains true.

To prove this we have only to show that (9.3) implies (9.1).

10. Uniqueness theorems. We turn next to the proof of

Theorem 15. If the function $F(x)$ is of class $C^{\infty}$ in the interval $0<x<\infty$ and satisfies the inequalities

$$
\left|F^{(k)}(x)\right|<\frac{M k !}{x^{k+1}} \quad(x>0 ; k=0,1, \cdots),
$$


then

$$
\lim _{k \rightarrow \infty} \int_{0}^{\infty} e^{-x t} L_{k, t}[F(x)] d t=F(x)
$$

That the integral (10.2) exists for $k=0,1,2, \cdots$ follows at once from the inequalities

$$
\begin{array}{r}
\left|L_{k, t}[F(x)]\right|<M \quad(t>0 ; k=0,1,2, \cdots), \\
\left|\int_{0}^{\infty} e^{-x t} L_{k, t}[F(x)] d t\right|<\int_{0}^{\infty} e^{-x t} M d t=\frac{M}{x} \quad(x>0) .
\end{array}
$$

If we set

we have

$$
\begin{aligned}
H_{k}(x) & =\int_{0}^{k / x} e^{-x t} L_{k, t}[F(x)] d t \\
I_{k}(x) & =\int_{k / x}^{\infty} e^{-x t} L_{k, t}[F(x)] d t
\end{aligned}
$$

$$
\int_{0}^{\infty} e^{-x t} L_{k, t}[F(x)] d t=H_{k}(x)+I_{k}(x),
$$

and it will be sufficient to show that

$$
\begin{array}{ll}
\lim _{k \rightarrow \infty} H_{k}(x)=F(x) & (x>0), \\
\lim _{k \rightarrow \infty} I_{k}(x)=0 & (x>0) .
\end{array}
$$

By the change of variable $u=k / t$ we obtain

$$
H_{k}(x)=(-1)^{k} \int_{x}^{\infty} \frac{u^{k-1}}{(k-1) !} e^{-k x / u} F^{(k)}(u) d u .
$$

On the other hand we have

$$
\begin{aligned}
(-1)^{k} & \int_{x}^{\infty} \frac{(u-x)^{k-1}}{(k-1) !} F^{(k)}(u) d u \\
& =\left.\frac{(-1)^{k}(u-x)^{k-1}}{(k-1) !} F^{(k-1)}(u)\right|_{x} ^{\infty}+(-1)^{k-1} \int_{x}^{\infty} \frac{(u-x)^{k-2}}{(k-2) !} F^{(k-1)}(u) d u .
\end{aligned}
$$

The first term on the right-hand side of this equation is zero since

$$
\lim _{u \rightarrow \infty} F^{(k-1)}(u) u^{k-1}=0,
$$

as we see from (10.1). By successive integration by parts we see that 
Hence

$$
F(x)=(-1)^{k} \int_{x}^{\infty} \frac{(u-x)^{k-1}}{(k-1) !} F^{(k)}(u) d u .
$$

$$
H_{k}(x)-F(x)=\int_{x}^{\infty} \frac{u^{k-1}}{(k-1) !}(-1)^{k} F^{(k)}(u)\left[e^{-k x / u}-\left(1-\frac{x}{u}\right)^{k-1}\right] d u .
$$

Again using (10.1),

$$
\left|H_{k}(x)-F(x)\right|<M k \int_{x}^{\infty} \frac{1}{u^{2}}\left|e^{-k x / u}-\left(1-\frac{x}{u}\right)^{k-1}\right| d u,
$$

or, by the change of variable $v=x / u$,

$$
\left|H_{k}(x)-F(x)\right|<\frac{M k}{x} \int_{0}^{1}\left|e^{-k v}-(1-v)^{k-1}\right| d v .
$$

We write

$$
e^{-k v}-(1-v)^{k-1}=\left[e^{-k v}-(1-v)^{k}\right]-v(1-v)^{k-1},
$$

and note that

$$
e^{-k v}-(1-v)^{k}>0
$$

as one sees by virtue of the familiar inequality

$$
e^{-x}>1-x
$$

Hence

$$
\begin{aligned}
\left|H_{k}(x)-F(x)\right| & <\frac{M k}{x}\left[\int_{0}^{1}\left[e^{-k v}-(1-v)^{k}\right] d v+\int_{0}^{1} v(1-v)^{k-1} d v\right] \\
& =\frac{M k}{x}\left[\frac{1-e^{-k}}{k}-\frac{1}{k+1}\right]+\frac{M k}{x} \frac{\Gamma(k) \Gamma(2)}{\Gamma(k+2)} \\
& =\frac{M}{x}\left[1-e^{-k}-\frac{k}{k+1}+\frac{1}{k+1}\right] .
\end{aligned}
$$

From this inequality it follows that

$$
\lim _{k \rightarrow \infty} H_{k}(x)=F(x)
$$

for all positive values of $x$.

For $I_{k}(x)$ we clearly have the inequality

$$
\left|I_{k}(x)\right|<\int_{k /:}^{\infty} M e^{-x t} d t=M e^{-k} / x \quad(x>0) .
$$


This shows that

$$
\lim _{k \rightarrow \infty} I_{k}(x)=0
$$

for all positive values of $x$, and the proof of the theorem is complete.

We now prove a companion theorem for the operator $S$.

THEOREM 16. If the function $f(x)$ is of class $C^{\infty}$ in the interval $0<x<\infty$, and if

$$
\left|\int_{x}^{\infty} \frac{u^{k}}{k !} f^{(k+1)}(u) d u\right|<M \quad(x>0 ; k=0,1, \cdots),
$$

then

$$
\lim _{k \rightarrow \infty} \int_{0}^{\infty} e^{-x t} d S_{k, t}[f(x)]=f(x)-f(\infty) \quad(x>0)
$$

where $S_{k, 0}[f(x)]$ is defined as $f(\infty)$.

The existence of the integral (10.3) for all $k$ enables us to employ Theorem 14 for an arbitrary value of $k$. Thus

$$
\begin{aligned}
& (-1)^{k} \frac{F^{(k)}(x) x^{k+1}}{k !} \\
& =f(\infty)+(-1)^{k+1} \int_{x}^{\infty} \frac{u^{k}}{k !} f^{(k+1)}(u) d u \quad(x>0 ; k=0,1, \cdots),
\end{aligned}
$$

where $F(x)=f(x) / x$. By (10.3) we have

$$
\left|F^{(k)}(x) x^{k+1} / k !\right| \leqq|f(\infty)|+M=N \quad(x>0 ; k=0,1, \cdots) .
$$

Hence we may apply Theorem 15 to $F(x)$ and obtain

$$
\lim _{k \rightarrow \infty} \int_{0}^{\infty} e^{-x t} L_{k, t}[F(x)] d t=F(x) .
$$

By Corollary 1 of Theorem 14,

$$
L_{k, t}[F(x)]=S_{k, t}[f(x)] \quad(t>0 ; k=0,1,2, \cdots) .
$$

The explicit expression for $S_{k, t}[f(x)]$ shows that

$$
\lim _{t \rightarrow 0} S_{k, t}[f(x)]=f(\infty) \text {. }
$$

An integration by parts, using (10.4), (10.5), and (10.6), gives

$$
\int_{0}^{\infty} e^{-x t} L_{k, t}[F(x)] d t=\frac{f(\infty)}{x}+\frac{1}{x} \int_{0}^{\infty} e^{-x t} d S_{k, t}[f(x)] \quad(x>0) .
$$


It is to be noted that we have not proved that $S_{k, t}[f(x)]$ is of bounded variation in $0 \leqq t \leqq R$. This is not necessary for the existence of the above Stieltjes integral. We have only to note that $e^{-x t}$ is a function of bounded variation in the interval $0 \leqq t \leqq R$ for every positive $x$ and $R$, and that $S_{k, t}[f(x)]$ is continuous in $0 \leqq t \leqq R$ (if defined as $f(\infty)$ at $t=0$ ). Allowing $k$ to become infinite in (10.7) we have

$$
\lim _{k \rightarrow \infty} \frac{1}{x} \int_{0}^{\infty} e^{-x t} d S_{k, t}[f(x)]=\frac{f(x)}{x}-\frac{f(\infty)}{x},
$$

from which the result of the theorem follows immediately.

Theorems 15 and 16 may be regarded as uniqueness theorems. The former shows at once that if $F_{1}(x)$ and $F_{2}(x)$ are two functions satisfying the inequalities (10.1) and such that

$$
L_{t}\left[F_{1}(x)\right]=L_{t}\left[F_{2}(x)\right],
$$

then $F_{1}(x)=F_{2}(x)$ for all positive values of $x$. For, the function

$$
\Phi(x)=F_{1}(x)-F_{2}(x)
$$

also satisfies inequalities (10.1). By Theorem 15

$$
\lim _{k \rightarrow \infty} \int_{0}^{\infty} e^{-x t} L_{k, t}[\Phi(x)] d t=\Phi(x)
$$

But by (10.8)

$$
\lim _{k \rightarrow \infty} L_{k, t}[\Phi(x)]=0
$$

Since

$$
\left|e^{-x t} L_{k, t}[\Phi(x)]\right|<e^{-x t} M,
$$

and since the function $M e^{-x t}$ is integrable with respect to $t$ on the infinite interval $(0, \infty)$ for every positive $x$, we may take the limit under the integral sign in (10.7) and obtain

$$
\Phi(x) \equiv 0 .
$$

In a similar way we can show that if $\Phi(x)$ is a function satisfying (10.3) and such that

$$
S_{t}[\Phi(x)] \equiv 0
$$

then $\Phi(x)$ is identically zero for all positive values of $x$. For, by Theorem 16,

$$
\lim _{k \rightarrow \infty} \int_{0}^{\infty} e^{-x t} d S_{k, t}[\Phi(x)]=\Phi(x)-\Phi(\infty) .
$$


But

$$
\int_{0}^{\infty} e^{-x t} d S_{k, t}[\Phi(x)]=-\Phi(\infty)+x \int_{0}^{\infty} e^{-x t} S_{k, t}[\Phi(x)] d t
$$

and since

$$
\left|S_{k, t} \Phi(x)\right|<N,
$$

we have, on allowing $k$ to become infinite,

$$
\Phi(x)-\Phi(\infty)=-\Phi(\infty) .
$$

The result is thus established.

11. Bernstein's theorem. By use of Theorem 16 we can now give a much simplified proof of a theorem of S. Bernstein.* As a preliminary result we prove

THEOREM 17. If the function $f(x)$ is completely monotonic in the interval $-\eta<x<\infty(\eta>0)$, then

$$
f(x)=\int_{0}^{\infty} e^{-x t} d \alpha(t)
$$

where the function $\alpha(t)$ is a non-decreasing function and the integral converges for $x>0$.

We recall that a function is completely monotonic in an interval $-\eta<x<\infty$ if it possesses derivatives of all orders there which satisfy the inequalities

$$
(-1)^{k} f^{(k)}(x) \geqq 0 \quad(-\eta<x<\infty) .
$$

We prove first that the limits

$$
\lim _{x \rightarrow \infty} x^{k} f^{(k)}(x) \quad(k=0,1,2, \cdots)
$$

exist. The result is obvious for $k=0$ since a completely monotonic function is non-negative and non-decreasing. Now form the function

$$
f(x)-x f^{\prime}(x) \text {. }
$$

It is clearly non-negative for $x>0$ and has a non-positive derivative

$$
\frac{d}{d x}\left(f(x)-x f^{\prime}(x)\right)=-x f^{\prime \prime}(x) .
$$

* S. Bernstein, Sur les fonctions absolument monotones, Acta Mathematica, vol. 52 (1929), p. 1. See also F. Hausdorf, Summationsmethoden und Momentfolgen, Mathematische Zeitschrift, vol. 9 (1921), pp. 280-299. 
It must therefore approach a limit as $x$ becomes infinite, and hence the function $x f^{\prime}(x)$ does also. We now proceed by induction. Suppose that the limit (11.1) exists for $k=0,1, \cdots, n$. We can prove that it also exists for $k=n+1$ by considering the function

$$
f(x)-x f^{\prime}(x)+\frac{x^{2} f^{\prime \prime}(x)}{2 !}-\cdots+\frac{(-1)^{n+1}}{(n+1) !} x^{n+1} f^{(n+1)}(x)
$$

whose derivative is

$$
(-1)^{n+1} x^{n+1} f^{(n+2)}(x) / n ! .
$$

The function (11.2) being non-negative non-increasing approaches a limit as $x$ becomes infinite. All terms except the last approach a limit by assumption, so that this last must also. This completes the induction.

By Corollary 2 of Theorem 14 we see that the integrals

$$
\int_{x}^{\infty} u^{k} f^{(k+1)}(u) d u \quad(k=0,1,2, \cdots)
$$

converge for positive $x$. Hence the function

$$
S_{k, t}[f(x)]=f(\infty)+\int_{k / t}^{\infty} \frac{u^{k}}{k !}(-1)^{k+1} f^{(k+1)}(u) d u
$$

is well defined for all positive values of $t$ and for all positive integers $k$. We define the function as $f(\infty)$ for $t=0$. Since $f(x)$ is completely monotonic for $x>0$, it is clear that the integrand of the integral (11.3) is non-negative. Hence $S_{k, t}[f(x)]$ is a non-negative non-decreasing function of $t$. Since the function $f^{(k+1)}(u)$ is continuous in the neighborhood of the origin, we have

$$
0 \leqq S_{k, t}[f(x)] \leqq f(\infty)+\int_{0}^{\infty} \frac{u^{k}}{k !}(-1)^{k+1} f^{(k+1)}(u) d u \quad(0 \leqq t<\infty) .
$$

But this integral is independent of $k$. In fact

$$
(-1)^{k+1} \int_{0}^{\infty} \frac{u^{k}}{k !} f^{(k+1)}(u) d u=f(0)-f(\infty),
$$

as one sees by successive integrations by parts. Hence

$$
0 \leqq S_{k, t}[f(x)] \leqq f(0) \quad(t \geqq 0 ; k=0,1,2, \cdots) .
$$

We are thus in a position to apply Theorem 16, for the boundedness of $S_{k, t}[f(x)]$ implies the condition (10.3). Hence

$$
f(x)=f(\infty)+\lim _{k \rightarrow \infty} \int_{0}^{\infty} e^{-x t} d S_{k, t}[f(x)] .
$$


Now by a theorem of E. Helly* it is possible to pick from the bounded sequence of functions $S_{k, t}[f(x)](k=0,1,2, \cdots)$ a sub-sequence $S_{j, t}[f(x)]$ which approaches a non-decreasing function $\beta(t)$ as $j$ becomes infinite. By the Helly-Bray theorem $\dagger$ it is permissible to take the limit under the integral sign so that we have

$$
f(x)=f(\infty)+\lim _{j \rightarrow \infty} \int_{0}^{\infty} e^{-x t} d S_{j, t}[f(x)]=f(\infty)+\int_{0}^{\infty} e^{-x t} d \beta(t) .
$$

Clearly

$$
\beta(0)=f(\infty) \geqq 0
$$

Hence if we define

$$
\begin{aligned}
\alpha(t) & =\beta(t) \\
\alpha(0) & =0,
\end{aligned}
$$

it is evident that $\alpha(t)$ remains a non-decreasing function and that

$$
f(x)=\int_{0}^{\infty} e^{-x t} d \alpha(t)
$$

The theorem is completely established. We. now prove the theorem of Bernstein:

THEOREM 18. A necessary and sufficient condition that $f(x)$ should be completely monotonic for $x>c$ is that

$$
f(x)=\int_{0}^{\infty} e^{-\dot{x} t} d \alpha(t),
$$

where the function $\alpha(t)$ is non-decreasing and the integral converges for $x>c$.

The sufficiency of the condition is established simply by noting that the function

$$
(-1)^{k} f^{(k)}(x)=\int_{0}^{\infty} e^{-x t} t^{k} d \alpha(t)
$$

must be non-negative for those values of $x$ which make the integral converge if $\alpha(t)$ is non-decreasing.

The necessity of the condition is easily established by use of Theorem 17 . The important distinction between our present hypothesis and that of Theorem 17 is that here we do not know the function $f(x)$ to be completely

* Helly, Über lineare Funktionaloperationen, Wiener Sitzungsberichte, vol. 121 (1921), p. 265.

† See, for example, G. C. Evans, The Logarithmic Potential, Discontinuous Dirichlet and Neumann Problems, Colloquium Publications, vol. 6, of the American Mathematical Society, 1927, p. 15. 
monotonic outside the interval in which the Laplace integral is to converge. We note first that the function $f(x+c+\eta)$ is completely monotonic for $x>-\eta$. Applying Theorem 17 we have

$$
f(x+c+\eta)=\int_{0}^{\infty} e^{-x t} d \beta_{\eta}(t),
$$

where $\beta_{\eta}(t)$ is non-decreasing and the integral converges for $x>0$. That is,

$$
f(x)=\int_{0}^{\infty} e^{-x t} e^{(c+\eta) t} d \beta_{\eta}(t)=\int_{0}^{\infty} e^{-x t} d \alpha_{\eta}(t),
$$

where

$$
\alpha_{\eta}(t)=\int_{0}^{t} e^{(c+\eta) u} d \beta_{\eta}(u)
$$

The integral (11.4) converges for $x>c+\eta$. This argument holds for each positive value of $\eta$ and appears to give various integral expressions for $f(x)$ corresponding to the various values of $\eta$ used. But since a function can have but a single Laplace integral representation, ${ }^{*}$ we see that $\alpha_{\eta}(t)$ is independent of $\eta$ and may be denoted by $\alpha(t)$. This is a non-decreasing function. Hence

$$
f(x)=\int_{0}^{\infty} e^{-x t} d \alpha(t)
$$

where the integral converges for $x>c+\eta$. Since $\eta$ was arbitrary, the integral converges for $x>c$ and the proof is complete.

12. Representation by absolutely convergent Laplace-Stieltjes integrals. We are now able to give by the present methods a much simplified proof of a theorem of the author. $\dagger$ We state it first in the slightly less general form:

THEOREM 19. A necessary and sufficient condition that the function $f(x)$ can be expressed as

$$
f(x)=\int_{0}^{\infty} e^{-x t} d \alpha(t)
$$

where $\alpha(t)$ is of bounded variation in the infinite interval $0 \leqq x<\infty$ is that $f(x)$ should be of class $C^{\infty}$ in that interval and that

* D. V. Widder, loc. cit., p. 705. We are assuming of course that the functions $\alpha_{\eta}(t), \beta_{\eta}(t)$ etc* are all normalized. That is,

$$
\alpha_{\eta}(0)=0, \quad\left[\alpha_{\eta}(t+)+\alpha_{\eta}(t-)\right] / 2=\alpha_{\eta}(t)
$$

$\dagger$ D. V. Widder, Necessary and sufficient conditions for the representation of a function as a Laplace integral, these Transactions, vol. 33 (1931), p. 851. 


$$
\int_{x}^{\infty} \frac{u^{k}}{k !}\left|f^{(k+1)}(u)\right| d u \leqq M \quad(x>0 ; k=0,1,2, \cdots) .
$$

Our present methods enable us to improve the proof of both the necessity and the sufficiency of the condition. We begin with the necessity. Suppose the function $f(x)$ to have the representation (12.1), the total variation of $\alpha(t)$ in the interval $(0, \infty)$ being equal to $M$. Then the integral (12.1) must converge absolutely for $x \geqq 0$. Denote the total variation of $\alpha(u)$ in the interval $0 \leqq u \leqq t$ by $V(t)$. Then $0 \leqq V(t) \leqq M$ for $0 \leqq t<\infty$. Set

Then

$$
g(x)=\int_{0}^{\infty} e^{-x t} d V(t) .
$$

$$
\left|f^{(k)}(x)\right|=\left|\int_{0}^{\infty} e^{-x t} t^{k} d \alpha(t)\right| \leqq \int_{0}^{\infty} e^{-x t} t^{k} d V(t)=(-1)^{k} g^{(k)}(x),
$$

and

$$
\begin{aligned}
\int_{x}^{\infty} \frac{u^{k}}{k !}\left|f^{(k+1)}(u)\right| d u \leqq(-1)^{k+1} \int_{x}^{\infty} \frac{u^{k}}{k !} g^{(k+1)}(u) d u \\
\\
\quad(x>0 ; k=0,1,2, \cdots) .
\end{aligned}
$$

Since $V(t)$ is a non-decreasing function, $g(x)$ is completely monotonic for $x>0$. Hence the integrals (12.3) surely converge for $x>0$ as the argument used in the proof of Theorem 17 shows. Integrating the right-hand member of (12.3) by parts, we obtain

$$
\begin{gathered}
\int_{k}^{\infty} \frac{u^{k}}{k !}\left|f^{(k+1)}(u)\right| d u \leqq g(x)-x g^{\prime}(x)+\frac{x^{2}}{2 !} g^{\prime \prime}(x)-\cdots+(-1)^{k} \frac{x^{k}}{k !} g^{(k)}(x)(x>0) \\
=\int_{0}^{\infty} e^{-x t}\left[1+x t+\frac{x^{2} t^{2}}{2 !}+\cdots+\frac{x^{k} t^{k}}{k !}\right] d V(t) .
\end{gathered}
$$

Again apply integration by parts to this last integral:

$$
\int_{x}^{\infty} \frac{u^{k}}{k !}\left|f^{(k+1)}(u)\right| d u \leqq \int_{0}^{\infty} e^{-x t} V(t) \frac{x^{k+1} t^{k}}{k !} d t \quad(x>0) .
$$

Finally, since $V(t) \leqq M$ we have

$$
\int_{x}^{\infty} \frac{u^{k}}{k !}\left|f^{(k+1)}(u)\right| d u \leqq M
$$

This completes the proof of the necessity of the condition.

We turn next to the proof of the sufficiency. The condition (12.2) on 
$f(x)$ enables us to show that $S_{k, t}[f(x)]$ is of bounded variation in the infinite interval $(0, \infty)$ and that the total variation of $f(x)$ in that interval has an upper bound independent of $k$. For, let $R$ be an arbitrary positive constant. Divide the interval $(0, R)$ into sub-intervals by points $t_{i}$ such that

$$
0=t_{0}<t_{1}<\cdots<t_{n}=R .
$$

Then we have

$$
\begin{aligned}
& \sum_{i=0}^{n-1}\left|S_{k, t_{i+1}}[f(x)]-S_{k, t_{i}}[f(x)]\right| \\
& \quad=\sum_{i=0}^{n-1}\left|\int_{k / t_{i+1}}^{\infty} \frac{u^{k}}{k !}(-1)^{k+1} f^{(k+1)}(u) d u-\int_{k / t_{i}}^{\infty} \frac{u^{k}}{k !}(-1)^{k+1} f^{(k+1)}(u) d u\right| \\
& \quad \leqq \sum_{i=0}^{n-1} \int_{k / t_{i+1}}^{k / t_{i}} \frac{u^{k}}{k !}\left|f^{(k+1)}(u)\right| d u=\int_{k / R}^{\infty} \frac{u^{k}}{k !}\left|f^{(k+1)}(u)\right| d u \leqq M .
\end{aligned}
$$

Since $M$ is independent of the manner of sub-division of the interval $(0, R)$ the function $S_{k, t}[f(x)]$ is of bounded variation in the interval and its total variation in that interval is at most $M$, a number independent of $k$ and of $R$. Hence the total variation of $S_{k, t}[f(x)]$ in $(0, \infty)$ is also at most equal to $M$ for all positive integers $k$.

Now by the theorem of Helly already employed in the proof of Theorem 17 we can pick from the sequence $S_{k, t}[f(x)]$ a sub-sequence $S_{j, t}[f(x)]$ which approaches a limit $\alpha(t)$ defined for $0<t<\infty$, whose total variation in that interval is at most $M$.

The condition (12.2) clearly implies the condition (10.3), so that we may apply Theorem 16 here to show that

$$
f(x)-f(\infty)=\lim _{j \rightarrow \infty} \int_{0}^{\infty} e^{-x t} d S_{j, t}[f(x)] .
$$

Since

$$
\left|S_{j, t}[f(x)]\right| \leqq|f(\infty)|+M
$$

we may use precisely the same argument as that used in the proof of Theorem 17 to show that we may take the limit under the sign of integration in (12.4). Thus

$$
f(x)=f(\infty)+\int_{0}^{\infty} e^{-x t} d \alpha(t) \quad(\alpha(0)=f(\infty)),
$$

or if $\alpha(0)$ is defined as zero,

$$
f(x)=\int_{0}^{\infty} e^{-x t} d \alpha(t) .
$$


In either case $\alpha(t)$ is of bounded variation in $(0, \infty)$ and the proof is complete.

We now prove the more general result by use of Theorem 19.

THEOREM 20. A necessary and sufficient condition that $f(x)$ can be expressed in the form

$$
f(x)=\int_{0}^{\infty} e^{-x t} d \alpha(t),
$$

where $\alpha(t)$ is of bounded variation in the interval $(0, R)$ for every positive $R$, the integral converging absolutely for $x>c$, is that $f(x)$ should be of class $C^{\infty}$ in the interval $c<x<\infty$ and that for every positive constant $\delta$ there should exist a constant $M_{8}$ such that

$$
\int_{x}^{\infty} \frac{u^{k}}{k !}\left|f^{(k+1)}(u+c+\delta)\right| d u \leqq M_{\delta} \quad(x>0 ; k=0,1,2, \cdots) .
$$

We prove first the necessity of the condition. Let $f(x)$ have the form (12.5). Then

$$
f(x+c+\delta)=\int_{0}^{\infty} e^{-x t} d \beta(t)
$$

where

$$
\beta(t)=\int_{0}^{t} e^{-(c+\delta) u} d \alpha(u) .
$$

The integral (12.7) converges absolutely for $x>-\delta$. The total variation of $\beta(u)$ in the interval $0 \leqq u \leqq t$ is

$$
\int_{0}^{t} e^{-(c+\delta) u} d V(u)
$$

where $V(t)$ is the total variation of $\alpha(u)$ in that interval. Hence the total variation of $\beta(u)$ in the interval $0 \leqq u<\infty$ is

$$
M_{\delta}=\int_{0}^{\infty} e^{-(c+\delta) u} d V(u) .
$$

This integral converges since (12.5) converges absolutely for $x=c+\delta$. Now applying Theorem 19 to the function $f(x+c+\delta)$ we get (12.6). This establishes the necessity of the condition.

Now assume that (12.6) holds. By Theorem 19 we have

$$
f(x+c+\delta)=\int_{0}^{\infty} e^{-x t} d \beta(t)
$$


where $\beta(t)$ is of bounded variation in the interval $0 \leqq t<\infty$. This integral consequently converges absolutely for $x \geqq 0$. Hence the integral

where

$$
f(x)=\int_{0}^{\infty} e^{-x t} d \alpha(t)
$$

$$
\alpha(t)=\int_{0}^{t} e^{(c+\delta) u} d \beta(u),
$$

converges absolutely for $x \geqq c+\delta$. This argument holds for each positive $\delta$. The function $\alpha(t)$ appears to depend on $\delta$. This is not the case, however, as one sees by again appealing to the uniqueness theorem for the representation of a function by a Laplace integral.* Since $\delta$ is arbitrary it follows that (12.5) converges absolutely for $x>c$ and the proof is complete.

It is natural to inquire what sort of condition is imposed on the function $f(x)$ by (12.6) if the absolute value signs are removed from the integrand and are applied instead to the integral itself. In this connection we prove

ThEOREM 21. A necessary and sufficient condition that the function $f(x)$ can be expressed in the form

$$
f(x)=x \int_{0}^{\infty} e^{-x t} \phi(t) d t
$$

where $\phi(t)$ is integrable in $(0, R)$ for every positive $R$, is uniformly bounded in $(0, \infty)$, and is such that the limit

$$
\lim _{t \rightarrow 0} \frac{1}{t} \int_{0}^{t} \phi(u) d u
$$

exists, is that a constant $M$ should exist for which

$$
\left|\int_{x}^{\infty} \frac{u^{k}}{k !} f^{(k+1)}(u) d u\right|<M \quad(x>0 ; k=0,1, \cdots) .
$$

We first establish the necessity of the condition. Set $F(x)=f(x) / x$. If $|\phi(t)|<N$ for $0 \leqq t<\infty$, then

$$
\left|\frac{F^{(k)}(x)}{k !} x^{k+1}\right|<N \quad(x>0 ; k=0,1,2, \cdots) .
$$

The hypothesis (12.8) implies that

$$
\int_{0}^{t} \phi(u) d u \sim a t
$$

* The function $\beta(u)$ of course depends on $\delta$. It is precisely this fact that makes it possible for $\alpha(t)$ to be independent of $\delta$. 
for a suitable constant $a$. From this it follows that

$$
\int_{0}^{t} u^{k} \phi(u) d u \sim a t^{k+1} /(k+1) \quad(t \rightarrow 0) .
$$

For, by virtue of (12.11), we have

$$
\int_{0}^{t}[\phi(u)-a] d u=o(t) \quad(t \rightarrow 0) .
$$

If we set

$$
\beta(t)=\int_{0}^{t} u^{k}[\phi(u)-a] d u,
$$

we must show that

$$
\beta(t)=o\left(t^{k+1}\right)
$$

By an integration by parts (12.12) is easily established.

This result enables us to show that

$$
(-1)^{k} F^{(k)}(x) \sim a k ! / x^{k+1} \quad(x \rightarrow \infty),
$$

or that

$$
I_{k}=\int_{0}^{\infty} e^{-x t} t^{k}[\phi(t)-a] d t=o\left(x^{-k-1}\right) \quad(x \rightarrow \infty) .
$$

Integration by parts gives

$$
\begin{aligned}
I_{k} & =x \int_{0}^{\infty} e^{-x t} \beta(t) d t \\
& =x \int_{0}^{\delta} e^{-x t} \beta(t) d t+x \int_{\delta}^{\infty} e^{-x t} \beta(t) d t .
\end{aligned}
$$

Using the relation (12.12) on the first integral on the right-hand side of this equation we obtain

$$
\left|I_{k}\right| \leqq \frac{(k+1) !}{x^{k+1}} \epsilon+x e^{-\left(x-x_{0}\right) \delta} \int_{\delta}^{\infty} e^{-x_{0} t}(N+a) t^{k+1} d t \quad\left(x>x_{0}>0\right) .
$$

But

$$
x e^{-\left(x-x_{0}\right) \delta}=o\left(x^{-k-1}\right) \quad(x \rightarrow \infty) .
$$

Hence it is evident that (12.13) is true. From this fact it follows at once that

$$
\lim _{x \rightarrow \infty} f(x)=a, \quad \lim _{x \rightarrow \infty} x^{k} f^{(k)}(x)=0 \quad(k=1,2, \cdots) .
$$


By Corollary 2 of Theorem 14 we have

$$
\frac{(-1)^{k}}{k !} F^{(k)}(x) x^{k+1}=f(\infty)+(-1)^{k+1} \int_{x}^{\infty} \frac{u^{k}}{k !} f^{(k+1)}(u) d u .
$$

That is,

$$
\left|\int_{x}^{\infty} \frac{u^{k}}{k !} f^{(k+1)}(u) d u\right| \leqq N+|f(\infty)|=M .
$$

This completes the proof of the necessity of the condition.

Conversely, suppose that (12.9) holds. Applying Theorem 14 we have

$$
\left|F^{(k)}(x) x^{k+1} / k !\right|<|f(\infty)|+M \quad(x>0 ; k=0,1,2, \cdots) .
$$

Then applying a theorem of the author* we see that

$$
F(x)=\frac{f(x)}{x}=\int_{0}^{\infty} e^{-x t} \phi(t) d t,
$$

where $\phi(t)$ is uniformly bounded in $(0, \infty)$. It remains only to show that (12.8) holds. We know that $f(\infty)$ exists since the integral (12.9) converges for $k=0$ by hypothesis. That is,

$$
F(x) \sim f(\infty) / x
$$

Since $\phi(t)$ is bounded we may apply a familiar Tauberian theorem $\dagger$ and conclude that

$$
\int_{0}^{t} \phi(u) d u \sim f(\infty) t \quad(t \rightarrow 0) .
$$

This completes the proof of the theorem.

\section{PART III}

\section{Applications}

13. Zeros of Laplace integrals. In this section we shall discuss the relation between the zeros of the integral

$$
f(x)=\int_{0}^{\infty} e^{-x t} d \alpha(t)
$$

and the changes of trend of the function $\alpha(t)$. In case $\alpha(t)$ has a continu-

* D. V. Widder, Necessary and sufficient conditions for the representation of a function as a Laplace integral, these Transactions, vol. 33 (1931), p. 873, Theorem 13.

† G. H. Hardy and J. E. Littlewood, On Tauberian theorems, Proceedings of the London Mathematical Society, vol. 30 (1930), p. 23. 
ous derivative $\alpha^{\prime}(t)$, E. Laguerre* proved that the number of zeros of $f(x)$ in the interval of convergence of (13.1) can not exceed the number of changes of sign of $\alpha^{\prime}(t)$. He obtained a similar result for the case in which (13.1) reduces to a Dirichlet series. We here extend the result to the general integral (13.1).

We first make a precise definition of the notion of change of trend. In this definition we use the term interval to include an interval of zero length. A function $\alpha(t)$ is increasing (or decreasing) in the zero interval $(a, a)$ if $\alpha(a+)>\alpha(a-)$ (or $\alpha(a+)<\alpha(a-))$.

DeFINITION. Let $\alpha(t)$ be a normalized function of bounded variation in the interval $0 \leqq t \leqq R$. Then $\alpha(t)$ has $n$ changes of trend in that interval if there exist points

$$
0=t_{0} \leqq t_{1} \leqq t_{2} \leqq \cdots \leqq t_{n+1}=R,
$$

with at most two consecutive $t_{i}$ equal, such that

$$
\begin{aligned}
& \alpha\left(t_{i+1}-\right) \neq \alpha\left(t_{i}+\right) \text { if } t_{i+1} \neq 0, t_{i} \neq R, \\
& \alpha(0) \neq \alpha(0+) \quad \text { if } \quad t_{0}=t_{1}=0, \\
& \alpha(R) \neq \alpha(R-) \quad \text { if } \quad t_{n+1}=t_{n}=R \text {; }
\end{aligned}
$$

(B) $\alpha(t)$ is alternately increasing and decreasing in the intervals

$$
\left(t_{0}, t_{1}\right),\left(t_{1}, t_{2}\right), \cdots,\left(t_{n}, t_{n+1}\right) .
$$

If a function has $n$ changes of trend in $(0, R)$ for every positive $R$ sufficiently large, we say that it has $n$ changes of trend in the infinite interval $(0, \infty)$. In particular if $\alpha(t)$ has a continuous derivative $\alpha^{\prime}(t)$ then $n$ is the number of changes in sign of $\alpha^{\prime}(t)$. On the other hand if $\alpha(t)$ is a step-function, so that (13.1) reduces to a Dirichlet series, $n$ is the number of changes of sign in the sequence of the coefficients. We now establish

THEOREM 22. If the function $\alpha(t)$ is of bounded variation in the interval $(0, R)$ for every positive $R$, and if it has $n$ changes of trend in the interval $(0, \infty)$, then the function

$$
f(x)=\int_{0}^{\infty} e^{-x t} d \alpha(t)
$$

has at most $n$ zeros in the interval of convergence of the integral.

By the definition of change of trend it is clear that the function

$$
\beta(x)=\int_{0}^{x}\left(t-t_{1}\right)\left(t-t_{2}\right) \cdots\left(t-t_{n}\right) d \alpha(t)
$$

\footnotetext{
* E. Laguerre, Oeuvres, vol. 1, p. 29.
} 
is monotonic in the interval $(0, \infty)$. Now form the function

$$
f^{*}(x)=\int_{0}^{\infty} e^{-x t} d \beta(t)=\int_{0}^{\infty} e^{-x t}\left(t-t_{1}\right)\left(t-t_{2}\right) \cdots\left(t-t_{n}\right) d \alpha(t) .
$$

Since $\beta(t)$ is monotonic it follows that $f^{*}(x)$ has no zeros in the interval of convergence of the integral (13.2). Set

$$
\left(t-t_{1}\right)\left(t-t_{2}\right) \cdots\left(t-t_{n}\right)=a_{n}(-t)^{n}+a_{n-1}(-t)^{n-1}+\cdots+a_{0} .
$$

Then

$$
f^{*}(x)=a_{n} f^{(n)}(x)+a_{n-1} f^{(n-1)}(x)+\cdots+a_{0} f(x) .
$$

If $f(x)$ had more than $n$ zeros, this linear differential expression would have at least one zero, as one sees by a generalized form of Rolle's theorem. $\dagger$ Since $f^{*}(x)$ has no zeros, our result is proved.

By use of our inversion formula we can now get a more exact relation between the number of zeros of $f^{(k)}(x)$ and the number of changes of trend in $\alpha(t)$. We prove

THEOREM 23. If the function $\alpha(t)$ is a normalized function of bounded variation with $n$ changes of trend in the interval $(0, R)$ for every sufficiently large positive $R$, and if $\alpha(0+)=\alpha(0)=0$, then

$$
f^{(k)}(x)=(-1)^{k} \int_{0}^{\infty} e^{-x t} t^{k} d \alpha(t)
$$

has exactly $n$ changes of sign in the interval of convergence of the integral for all $k$ sufficiently large.

Before proving the theorem we point out that the restriction $\alpha(0+)=\alpha(0)$ is a necessary one. If it were omitted, $\alpha(t)$ could be defined as

$$
\begin{array}{llr}
\alpha(0)=0, & \alpha(t)=1 & (0<t<1), \\
\alpha(1)=\frac{1}{2}, & \alpha(t)=0 & (1<t<\infty),
\end{array}
$$

a function with one change of trend. Yet the derivatives of

$$
1-e^{-x}=\int_{0}^{\infty} e^{-x t} d \alpha(t)
$$

have no change of sign, no matter how high the order.

To prove the theorem consider the points $t_{0}, t_{1}, \cdots, t_{n}$ whose existence is

$\dagger$ G. P6lya, On the mean-value theorem corresponding to a given linear homogeneous differential equation, these Transactions, vol. 24 (1922), pp. 312-324.

D. V. Widder, A general mean-value theorem, these Transactions, vol. 26 (1924), pp. 385-394. Since the coefficients $a_{i}$ are constants, the property $W$ is satisfied in any interval. 
guaranteed by the foregoing definition. Consider two adjoining intervals $\left(t_{i-1}, t_{i}\right)$ and $\left(t_{i}, t_{i+1}\right)$. Suppose that $\alpha(t)$ is increasing in the first and decreasing in the second. We show first that points $\xi, \eta, \zeta$ exist such that

$$
\alpha(\xi)<\alpha(\eta)>\alpha(\zeta), \quad t_{i-1} \leqq \xi<\eta<\zeta \leqq t_{i+1} .
$$

We consider several cases.

CASE I. $t_{i-1} \neq t_{i}, t_{i} \neq t_{i+1}$. In this case $\alpha(t)$ has at least one point of increase in $t_{i-1} \leqq t \leqq t_{i}$. Hence we can find $\xi$ and $\eta^{\prime}$ such that

$$
\alpha(\xi)<\alpha\left(\eta^{\prime}\right) \quad\left(t_{i-1} \leqq \xi<\eta^{\prime} \leqq t_{i}\right) .
$$

Since $\alpha(t)$ has at least one point of decrease in $t_{i} \leqq t \leqq t_{i+1}$ we can determine $\eta^{\prime \prime}$ and $\zeta$ such that

$$
\alpha\left(\eta^{\prime \prime}\right)>\alpha(\zeta) \quad\left(t_{i} \leqq \eta^{\prime \prime}<\zeta \leqq t_{i+1}\right) .
$$

Choose $\eta$ equal to $\eta^{\prime}$ or $\eta^{\prime \prime}$ so that

$$
\begin{aligned}
& \alpha(\eta) \geqq \alpha\left(\eta^{\prime}\right), \\
& \alpha(\eta) \geqq \alpha\left(\eta^{\prime \prime}\right) .
\end{aligned}
$$

Then clearly (13.3) is satisfied.

CASE II. $t_{i-1} \neq t_{i}, t_{i}=t_{i+1}$. Choose $\xi$ and $\eta^{\prime}$ as in Case I. By B of the definition we see that $\alpha\left(t_{i+1}+\right)-\alpha\left(t_{i}-\right)<0$. Hence we can determine $\eta^{\prime \prime}$ such that

$$
\alpha\left(\eta^{\prime \prime}\right)>\alpha\left(t_{i+1}\right)=\frac{\alpha\left(t_{i+1}+\right)+\alpha\left(t_{i}-\right)}{2} .
$$

Choose $\eta$ as in Case I and $\zeta=t_{i+1}$. Then (13.3) is satisfied.

CASE III. $t_{i-1}=t_{i}, t_{i} \neq t_{i+1}$. The treatment of this case is similar to that of Case II and is omitted.

It is to be noted that $t_{1} \neq t_{0}$ since $\alpha(0+)=\alpha(0)$. Hence $\xi>0$ if $i=1$.

Now choose a positive number $\epsilon$ so small that

$$
\alpha(\xi)+\epsilon<\alpha(\eta)-\epsilon<\alpha(\zeta)+\epsilon .
$$

By Theorem 2 we can determine an integer $k_{0}$ so large that

$$
\left|S_{k, t}[f(x)]-\alpha(t)\right|<\epsilon \quad\left(t=\xi, \eta, \zeta ; k>k_{0}\right) .
$$

Hence

$$
S_{k, \xi}[f(x)]<S_{k, \eta}[f(x)]>S_{k, \xi}[f(x)] .
$$

Since $S_{k, t}[f(x)]$ is a function of class $C^{\prime}$ at least, it follows that it has at least one maximum in the interval $t_{i-1}<t<t_{i+1}$, where its derivative vanishes. A similar proof applies if $\alpha(t)$ is decreasing in $\left(t_{i-1}, t_{i}\right)$ and increasing in $\left(t_{i}, t_{i+1}\right)$. 
Since there are but a finite number of intervals $\left(t_{i}, t_{i+1}\right)$ we can determine $k_{0}$ so large that for $k>k_{0}$ the function $S_{k, t}$ will have at least one

$$
\begin{aligned}
& \text { maximum (minimum) in }\left(t_{0}, t_{2}\right), \\
& \text { minimum (maximum) in }\left(t_{1}, t_{3}\right), \\
& \text { maximum (minimum) in }\left(t_{2}, t_{4}\right), \\
& \ldots
\end{aligned}
$$

It thus becomes clear that the derivative of $S_{k, t}[f(x)]$ with respect to $t$ will change sign at least $n$ times in $(0, \infty)$. That is, the function

$$
\frac{k^{k}}{(k-1) !} \frac{1}{t^{k+2}} f^{(k+1)}\left(\frac{k}{t}\right)
$$

and hence also $f^{(k+1)}(x)$, must change sign at least $n$ times in $(0, \infty)$. By Theorem 22 we see that $f^{(k+1)}(x)$ must change sign exactly $n$ times in that interval and the theorem is completely established.

CoRollary 1. If $\alpha(t)$ has a maximum (minimum)* at a point $t_{0}$, then for $k$ sufficiently large $f^{(k)}(x)$ will have a change of sign at a point $x_{k}$ such that

$$
\lim _{k \rightarrow \infty} \frac{k}{x_{k}}=t_{0}
$$

Let $\epsilon$ be an arbitrary positive number. We must show that there corresponds a number $k_{0}$ such that $f^{(k)}(x)$ will have a zero $x_{k}$ for which

$$
\left|\frac{k}{x_{k}}-t_{0}\right|<\epsilon
$$

or that $f^{(k)}(k / t)$ will have a zero in the interval $t_{0}-\epsilon<t<t_{0}+\epsilon$. This follows from the inequalities

$$
S_{k, t_{0}-\epsilon}[f(x)]<S_{k, t_{0}}[f(x)]>S_{k, t_{0}+\epsilon}[f(x)]
$$

precisely as in the proof of the theorem.

Corollary 2. If

$$
\begin{aligned}
& f(x)=a_{1} e^{-\lambda_{1} x}+a_{2} e^{-\lambda_{2} x}+a_{3} e^{-\lambda_{8} x}+\cdots, \\
& 0<\lambda_{1}<\lambda_{2}<\lambda_{3}<\cdots, \quad \lim _{m \rightarrow \infty} \lambda_{m}=\infty,
\end{aligned}
$$

and if the sequence $a_{1}, a_{2}, a_{3}, \cdots$ has $n$ changes of sign, $f^{(k)}(x)$ will have exactly $n$

* We do not require that $\alpha(t)$ should be continuous at $t_{0}$. We must, however, have a maximum (minimum) in the strict sense. 
changes of sign in the interval of convergence of the series for all $k$ sufficiently large.

COROLlary 3. If $\alpha(t)$ has $n_{R}$ changes of trend in $(0, R)$ and if

$$
\lim _{R \rightarrow \infty} n_{R}=\infty,
$$

then the number of changes of sign of $f^{(k)}(x)$ in the interval of convergence of the integral becomes infinite with $k$.

Here we introduce the further

Defrnition. Let $\phi(t)$ be integrable in $(0, R)$. It has $n$ changes of sign in that interval if the function

$$
\alpha(t)=\int_{0}^{t} \phi(y) d y
$$

has $n$ changes of trend there.

For example, if $(-1)^{i} \phi(t) \geqq 0$ almost everywhere in $\left(t_{i}, t_{i+1}\right)$, the sign $>$ holding for a set of positive measure $(i=0,1,2, \cdots, n)$, then the conditions of the definition are satisfied.

COROLLARY 4. If $\phi(t)$ is integrable in $(0, R)$ and has $n$ changes of sign there for every positive $R$, then the function

$$
f^{(k)}(x)=(-1)^{k} \int_{0}^{\infty} e^{-x t} t^{k} \phi(t) d t
$$

has $n$ changes of sign in the interval of convergence of the integral for all $k$ sufficiently large.

This result includes as a special case a result which the author stated earlier without proof.* The increased generality of the present result is noteworthy.

In case the function $\phi(t)$ of Corollary 4 has its changes of sign at points in the neighborhood of which it is different from zero, our inversion formula enables us to locate the positions of the changes of sign of $\phi(t)$ if we know the positions of the changes of sign of $f^{(k)}(x)$. We first make exact the notion of change of sign at a point.

Definition. Let $\phi(t)$ be defined in the interval $(0, R)$. Then it has a change of sign at a point $t=t_{i}\left(0<t_{i}<R\right)$ if for all positive $\epsilon$ sufficiently small

or

$$
\phi\left(t_{i}-\epsilon\right)>0, \phi\left(t_{i}+\epsilon\right)<0,
$$

$$
\phi\left(t_{i}-\epsilon\right)<0, \phi\left(t_{i}+\epsilon\right)>0 .
$$

\footnotetext{
* See the author's Proceedings article cited in the Introduction, Theorem 5.
} 
We now establish

THEOREM 24. If $\phi(t)$ is integrable in $(0, R)$ for every positive $R$, the integral

$$
f(x)=\int_{0}^{\infty} e^{-x t} \phi(t) d t
$$

converging for some value of $x$, and if $\phi(t)$ has a change of sign at a point $t_{0}$, then for $k$ sufficiently large $f^{(k)}(x)$ will have a change of sign at a point $x_{k}$ such that

$$
\lim _{k \rightarrow \infty} \frac{k}{x_{k}}=t_{0}
$$

Given an arbitrary positive $\epsilon$ we wish to show that we can find an integer $k_{0}$ such that for $k>k_{0}$ the function $f^{(k)}(x)$ will have a change of sign $x_{k}$ such that

$$
\left|\frac{k}{x_{k}}-t_{0}\right|<\epsilon
$$

or that the function $f^{(k)}(k / t)$ will have a change of sign between $t_{0}-\epsilon$ and $t_{0}+\epsilon$ for all $k>k_{0}$. By Theorem 4 the function $L_{k, t}[f(x)]$ approaches $f(x)$ almost everywhere in $(0, \infty)$. Choose a point $\eta$ in the interval $t_{0}<t<t_{0}+\epsilon$ and a point $\xi$ in the interval $t_{0}-\epsilon<t<t_{0}$ such that

$$
\begin{aligned}
& \lim _{k \rightarrow \infty} L_{k, \xi}[f(x)]=\phi(\xi), \\
& \lim _{k \rightarrow \infty} L_{k, \eta}[f(x)]=\phi(\eta) .
\end{aligned}
$$

But

$$
\phi(\xi) \neq 0, \phi(\eta) \neq 0, \phi(\xi) \phi(\eta)<0 .
$$

Hence we can determine $k_{0}$ so large that for $k>k_{0}$

$$
L_{k, \xi}[f(x)]>\frac{\phi(\xi)}{2}>0, L_{k, \eta}[f(x)]<\frac{\phi(\eta)}{2}<0,
$$

or else

$$
L_{k, \xi}[f(x)]<\frac{\phi(\xi)}{2}<0, L_{k, \eta}[f(x)]>\frac{\phi(\eta)}{2}>0
$$

In either case the continuous function $L_{k, t}[f(x)]$ must vanish between $\xi$ and $\eta$. But this function vanishes only when $f^{(k)}(k / t)$ vanishes, so that the theorem is established. We point out that the theorem could also be derived by use of Corollary 1 to Theorem 22. 
CoRollary. If $\phi(t)$ is continuous in $(0, \infty)$ and has changes of sign at the points $t_{i}$

$$
0<t_{1}<t_{2}<\cdots<t_{n}
$$

and at no others, then $f^{(k)}(x)$ has exactly $n$ changes of sign for $k$ sufficiently large at points

and

$$
x_{1, k}>x_{2, k}>\cdots>x_{n, k},
$$

$$
\lim _{k \rightarrow \infty} \frac{x_{i, k}}{k}=\frac{1}{t_{i}} \quad(i=1,2, \cdots, n) .
$$

Included in this Corollary is a result stated earlier by the author.*

We now illustrate the theory by an example. Take

Then

$$
\phi(t)=(t-a)(t-b) \quad(0<a<b) .
$$

$$
\begin{aligned}
f(x) & =\frac{2}{x^{3}}-\frac{(a+b)}{x^{2}}+\frac{a b}{x} \\
f^{(k)}(x) & =\frac{(k+2) !}{x^{k+3}}-\frac{(a+b)(k+1) !}{x^{k+2}}+\frac{a b k !}{x^{k+1}} .
\end{aligned}
$$

Simple computations show that

$$
\begin{aligned}
& x_{1, k}=\left\{(a+b)(k+1)+\left[(a+b)^{2}(k+1)^{2}-4 a b(k+1)(k+2)\right]^{1 / 2}\right\} /(2 a b), \\
& x_{2, k}=\left\{(a+b)(k+1)-\left[(a+b)^{2}(k+1)^{2}-4 a b(k+1)(k+2)\right]^{1 / 2}\right\} /(2 a b) .
\end{aligned}
$$

These roots will be real if $k$ is sufficiently large. It must be so large that

$$
\frac{k+1}{k+2}>\frac{4 a b}{(a+b)^{2}} .
$$

The right-hand side of this inequality is less than unity if $a \neq b$, whereas the left-hand side approaches unity as $k$ becomes infinite. It is clear that

$$
\lim _{k \rightarrow \infty} \frac{x_{1, k}}{k}=\frac{1}{a}, \quad \lim _{k \rightarrow \infty} \frac{x_{2, k}}{k}=\frac{1}{b} .
$$

This example shows that $f^{(k)}(x)$ may have a smaller number of changes of sign than $\phi(t)$ for small values of $k$. For example, if $a=9, b=10$, the function $f^{(k)}(x)$ has no zeros for $k<359$, has two zeros for $k>359$.

As a further application of Theorem 23 we prove

* Theorem 6 of the Proceedings article cited above. 
TheOREM 25. Let the series

$$
f(z)=\sum_{m=1}^{\infty} a_{m} z^{m}
$$

have radius of convergence $R$, and let the sequence of real coefficients $a_{1}, a_{2}, \ldots$ have $n$ changes of sign. Then the function

$$
f_{k}(z)=\sum_{m=1}^{\infty} a_{m}(m)^{k} z^{m}
$$

will have exactly $n$ changes of sign in the interval $0<z<R$ for all $k$ sufficiently large.

Set $z=e^{-x}$ in the given power series. We thus obtain a Dirichlet series convergent for $x>\log (1 / R)$, to which we apply Corollary 2 of Theorem 23 . Its coefficients have exactly $n$ changes of sign. Hence its $k$ th derivative will have exactly $n$ changes of sign in the interval $\log (1 / R)<x<\infty$ for all $k$ sufficiently large. Replacing $x$ by $\log (1 / z)$ we have the result stated.*

We may also obtain a similar result concerning factorial series.

TheOREM 26. Let the sequence $a_{1}, a_{2}, \cdots$ have $n$ changes of sign, and let the series

$$
f_{l}(x)=\sum_{m=1}^{\infty} \frac{a_{m} m ! m^{l}}{x(x+1) \cdots(x+m)}
$$

converge for $x>0$. Then it is possible to determine a number $l_{0}$ such that for any fixed $l>l_{0}$ the function $f_{l}^{(k)}(x)$ will have $n$ changes of sign in the interval $0<x<\infty$ for all $k$ sufficiently large.

Since the series (13.5) converges we have $\dagger$

$$
f_{l}(x)=\int_{0}^{\infty} e^{-x t} \phi_{l}(t) d t
$$

where

$$
\phi_{l}(t)=\sum_{m=1}^{\infty} a_{m} m^{l}\left(1-e^{-t}\right)^{m-1} .
$$

The series (13.6) converges for $0<t<\infty$, or the series

$$
\psi_{l}(z)=\sum_{m=1}^{\infty} a_{m} m^{l} z^{m-1}
$$

* Cf. P6lya and Szegö, A ufgaben und Lehrsätze aus der Analysis, vol. 2 (1925), p. 44, No. 44.

† D. V. Widder, I, p. 739. 
converges for $0<z<1$. By Theorem 25 the function $\psi_{l}(z) z$ will have exactly $n$ changes of sign in $0<z<1$ if $l$ is greater than some number $l_{0}$. The same will be true of $\psi_{l}(z)$ and hence of $\phi_{l}(t)$ in $0<t<\infty$. We have now only to apply Corollary 3 of Theorem 23 to (13.7) to obtain the result stated.*

14. Special inversion formulas. We conclude Part III with several specific inversion formulas which, although they are immediate consequences of the general theory, are nevertheless worthy of separate statement. The first has to do with functions of the form

$$
f(x)=\int_{0}^{\infty} \frac{d \alpha(t)}{x+t}
$$

considered by Stieltjes. He gave a complex inversion formula when $\alpha(t)$ is an increasing function. $\dagger$ Our present methods enable us to give the following simple real solution of the Stieltjes problem.

THEOREM 27. If the function $\alpha(t)$ is of bounded variation in the infinite interval $(0, \infty)$, and if

$$
f(x)=\int_{0}^{\infty} \frac{d \alpha(t)}{x+t}
$$

then

$$
\frac{\alpha(t+)+\alpha(t-)}{2}=S_{t}\left[L_{v}[f(x)]\right] \quad(t>0) .
$$

To prove this result set

$$
\phi(y)=\int_{0}^{\infty} e^{-y t} d \alpha(t) .
$$

Since $\alpha(t)$ is of bounded total variation in $(0, \infty)$, this integral converges absolutely for $0 \leqq y<\infty$. It follows that the integral (14.1) converges for $x>0$, that $f(x)$ is analytic in the whole complex $x$-plane with the negative real axis removed, and that

$$
f(x)=\int_{0}^{\infty} e^{-x y} \phi(y) d y .
$$

This integral converges for $x>0$. But

$$
\frac{\alpha(t+)+\alpha(t-)}{2}=S_{t}[\phi(y)]
$$

* Compare P6lya and Szegö, loc. cit., vol. 2, p. 51, No. 84.

† See, for example, O. Perron, Die Lehre von den Kettenbrüchen, 1929, p. 372. 
and

$$
\phi(y)=L_{y}[f(x)]
$$

by Theorems 2 and 3 respectively. Combining these two results we have (14.2).

Another application of Theorem 2 to Dirichlet series is contained in THEOREM 28. If

$$
\begin{gathered}
f(x)=a_{1} e^{-\lambda_{1} x}+a_{2} e^{-\lambda_{2} x}+\cdots, \\
0<\lambda_{1}<\lambda_{2}<\cdots, \quad \lim _{n \rightarrow \infty} \lambda_{n}=\infty,
\end{gathered}
$$

the series converging for $x>c$, then

$$
a_{n}=\lim _{k \rightarrow \infty}(-1)^{k+1} \int_{2 k /\left(\lambda_{n}+\lambda_{n+1}\right)}^{2 k /\left(\lambda_{n-1}+\lambda_{n}\right)} \frac{u^{k}}{k !} f^{(k+1)}(u) d u \quad(n=1,2, \cdots),
$$

where $\lambda_{0}$ is defined as zero.

This follows since

$$
a_{n}=S_{\left(\lambda_{n}+\lambda_{n+1}\right) / 2}[f(x)]-S_{\left(\lambda_{n-1}+\lambda_{n}\right) / 2}[f(x)] .
$$

In a similar way we may obtain the coefficients of a series in powers of $1 / x$ in terms of the function it represents.

THEOREM 29. If

$$
f(x)=\frac{a_{0}}{x}+\frac{a_{1}}{x^{2}}+\frac{a_{2}}{x^{3}}+\cdots,
$$

the series converging for $x>c$, then

$$
a_{n}=\left\{\lim _{k \rightarrow \infty} \frac{d^{n}}{d t^{n}} L_{k, t}[f(x)]\right\}_{t=0}
$$

This follows since $f(x)$ can be represented as a Laplace integral*

where

$$
f(x)=\int_{0}^{\infty} e^{-x t} \phi(t) d t
$$

$$
\phi(t)=a_{0}+a_{1} t+\frac{a_{2} t^{2}}{2 !}+\frac{a_{3} t^{3}}{3 !}+\cdots .
$$

An application of Theorem 12 now gives the result.

Finally, the coefficients of a factorial series can also be determined in

* See for example, D. V. Widder, I, p. 728. 
terms of the function which it represents.

THEOREM 30. If

$$
f(x)=\frac{a_{0}}{x}+\frac{a_{1}}{x(x+1)}+\frac{a_{2}}{x(x+1)(x+2)}+\cdots,
$$

the series converging for $x>c$, then

$$
a_{n}=\left\{\lim _{k \rightarrow \infty} \frac{d^{n}}{d t^{n}} L_{k, \log (1 /(1-t))}[f(x)]\right\}_{t=0} .
$$

The proof is similar to that of Theorem 29 and is omitted.

PART IV

\section{COMPLEX VARIABLE}

15. Generating function analytic at infinity. In previous work we have regarded the generating function $f$ and the determining function $\phi$ as real functions of the real variable. Let us now suppose that both are functions of the complex variable. Set

$$
z=x+i y, s=\sigma+i \tau
$$

and write

$$
f(s)=\int_{0}^{\infty} e^{-s z} \phi(z) d z .
$$

We are still supposing that the path of integration is along the positive real axis. If the integral converges for some value of $s$ we easily see by breaking $\phi(x)$ into its real and imaginary parts that

$$
L_{x}[f(s)]=\phi(x)
$$

for all real positive values of $x$. It is natural to inquire if this formula still holds when $x$ is replaced by the complex variable $z$. In other words, will our inversion formula hold off the real axis? We shall be able to show that it holds in the half-plane $x>0$ if the function $\phi(z)$ is analytic there. In the present section we shall assume in addition that $f(s)$ is analytic at infinity and vanishes there. We recall that such a function can be expressed in the form (15.1). If

$$
f(s)=\sum_{n=0}^{\infty} \frac{a_{n}}{s^{n+1}},
$$

then 


$$
\phi(z)=\sum_{n=0}^{\infty} \frac{a_{n} z^{n}}{n !}
$$

and $\phi(z)$ is entire.*

We now establish

THEOREM 31. If $f(s)$ is analytic at infinity and vanishes there, then $L_{z}[f(s)]$ exists for all complex $z$ and defines an entire function $\phi(z)$ such that

$$
f(s)=\int_{0}^{\infty} e^{-s z} \phi(z) d z,
$$

the integral converging in some half-plane $\sigma>\sigma_{c}$.

Since $f(s)$ has the representation (15.2), the series converging in some neighborhood of infinity, there exist numbers $M$ and $\rho$ such that

$$
\left|a_{n}\right|<M \rho^{n} \quad(n=0,1,2, \cdots) .
$$

Simple computation gives

$$
L_{k, z}[f(s)]=\sum_{n=0}^{\infty} \frac{(n+k) !}{n !} \frac{a_{n} z^{n}}{k^{n} k !} \quad(z<1 / \rho) .
$$

It will now be shown that the series (15.4), whose terms are to be regarded as functions of the two variables $k$ and $z$, converges uniformly in the region $|z| \leqq l, k \geqq k_{0}$, where $l$ is an arbitrary positive constant and $k_{0}$ is a suitably chosen positive integer. We note that

$$
\frac{(n+k) !}{k^{n} k !}=\left(1+\frac{1}{k}\right)\left(1+\frac{2}{k}\right) \cdots\left(1+\frac{n}{k}\right)
$$

is a decreasing function of $k$ for any positive integer $n$. Having chosen $l$ arbitrarily we choose the integer $k_{0}$ greater than $l \rho$. We thus have

$$
\sum_{n=0}^{\infty} \frac{(n+k) !}{k^{n} k !} \frac{a_{n} z^{n}}{n !} \ll \sum_{n=0}^{\infty} \frac{\left(n+k_{0}\right) !}{k_{0}{ }^{n} k_{0} !} \frac{M \rho^{n} l^{n}}{n !} .
$$

Since the convergent dominant series is independent of $k$ and of $z$, the uniform convergence of (15.4) is established. Consequently we may take the limit of the series (15.4) term by term as $k$ becomes infinite for any fixed $z$ whose modulus is $\leqq l$ provided that the limit of the general term exists. But

It follows that

$$
\lim _{k \rightarrow \infty} \frac{(n+k) !}{n !} \frac{a_{n} z^{n}}{k^{n} k !}=\frac{a_{n} z^{n}}{n !} .
$$

\footnotetext{
* See, for example, D. V. Widder, I, p. 728.
} 


$$
L_{z}[f(s)]=\lim _{k \rightarrow \infty} L_{k, z}[f(s)]=\sum_{n=0}^{\infty} \frac{a_{n} z^{n}}{n !}=\phi(z)
$$

for $|z| \leqq l$. Since $l$ was arbitrary, the theorem is completely established.

COROLLARY. Under the conditions of the theorem,

$$
\lim _{k \rightarrow \infty} L_{k, z}[f(s)]=\phi(z)
$$

uniformly in any closed region of the z-plane.

This follows at once from the fact that the series (15.4) converged uniformly in $z$ as well as in $k$.

16. Determining function analytic in a half-plane. We now turn to the case in which the determining function is analytic in the half-plane. We shall show that in this case our inversion formula is valid throughout the halfplane. The result to be proved is

TheOREM 32. Let the function $\phi(z)$ be analytic in the half-plane $x>0$ and let the integral

$$
f(s)=\int_{0}^{\infty} e^{-s z} \phi(z) d z
$$

converge for some value of $s$. Then

$$
\lim _{k \rightarrow \infty} L_{k, z}[f(s)]=\phi(z)
$$

uniformly in any closed region in the half-plane $x>0$.

It is to be noted that we are not assuming that $\phi(z)$ is analytic at the origin. Thus our proof will apply to such functions as $z^{-1 / 2}$. This degree of generality is reflected in a corresponding complication in the proof.

We obtain at once the following integral representation of $L_{k, z}[f(s)]$,

$$
L_{k, z}[f(s)]=\frac{1}{k !}\left(\frac{k}{z}\right)^{k+1} \int_{0}^{\infty} e^{-k u / z} u^{k} \phi(u) d u .
$$

Here $u$ is for the present a real variable. Later we shall alter the path of integration and $u$ will be a complex variable. Set $z=\rho e^{i \psi}$ and $u=r e^{i \theta}$. Let $D$ be an arbitrary closed region in the half-plane $x>0$. We can determine positive constants $\rho_{0}, \rho_{1}$ and $\psi_{1}<\pi / 2$ such that for all points $z$ of $D$ we have $\rho_{0} \leqq \rho \leqq \rho_{1}$, $|\psi|<\psi_{1}$. Now consider the function

$$
\left|e^{-u / z} u / z\right|=(r / \rho) \exp [(-r / \rho) \cos (\theta-\psi)] .
$$

As $z$ varies in $D$ and $u$ varies along the positive real axis we have 


$$
(r / \rho) \exp [(-r / \rho) \cos \psi]<\left(r / \rho_{0}\right) \exp \left[\left(-r / \rho_{1}\right) \cos \psi_{1}\right]
$$

The right-hand side of this inequality is independent of the value of $z$ in $D$ and tends to zero as $r$ becomes infinite or as $r$ approaches zero. Consequently we can determine a positive number $\delta<\rho_{0}$ and a positive number $\Delta>\rho_{1}$ both independent of $z$ in $D$ such that

$$
\left|e^{-u / z} u / z\right|<e^{-1}
$$

for $r \leqq \delta$ and for $r \geqq \Delta$.

We can now show that

$$
\begin{aligned}
& \lim _{k \rightarrow \infty} \frac{1}{k !}\left(\frac{k}{z}\right)^{k+1} \int_{\Delta}^{\infty} e^{-k u / z} u^{k} \phi(u) d u=0 \\
& \lim _{k \rightarrow \infty} \frac{1}{k !}\left(\frac{k}{z}\right)^{k+1} \int_{0}^{\delta} e^{-k u / z} u^{k} \phi(u) d u=0
\end{aligned}
$$

uniformly in $D$. Set

$$
\alpha(u)=\int_{0}^{u} \phi(r) d r
$$

for positive real values of $u$. Then constants $M$ and $g$ exist such that

$$
|\alpha(u)|<M e^{o u} \quad(0 \leqq u<\infty) .
$$

Hence

$$
\begin{aligned}
\frac{1}{k !}\left(\frac{k}{z}\right)^{k+1} \int_{\Delta}^{\infty} e^{-k u / z} u^{k} \phi(u) d u & =-\alpha(\Delta) \frac{1}{k !}\left(\frac{k}{z}\right)^{k+1} e^{-k \Delta / z} \Delta^{k} \\
& \quad-\frac{1}{(k-1) !}\left(\frac{k}{z}\right)^{k+1} \int_{\Delta}^{\infty} \alpha(u) e^{-k u / z} u^{k-1}\left[1-\frac{u}{z}\right] d u
\end{aligned}
$$

for $k$ sufficiently large. The first term on the right-hand side of this equation satisfies the inequality

$$
|\alpha(\Delta)|\left|e^{-\Delta / z} \frac{\Delta}{z}\right|^{k} \frac{k^{k+1}}{k !} \rho^{-1} \leqq|\alpha(\Delta)| \frac{k^{k+1}}{k !} l^{k} \rho_{0}^{-1}
$$

where $l<e^{-1}$, as one sees by (16.1). The dominant function is independent of $z$ in $D$ and approaches zero with $1 / k$.

The second term on the right-hand side of (16.5) is in modulus at most equal to

$$
\frac{k^{k+1}}{(k-1) !} \frac{1}{\rho_{0}^{2}} l^{k-k_{0}} \int_{\Delta}^{\infty}|\alpha(u)| e^{-\left\{k_{0} u \cos \psi_{1}\right] / \rho_{1}}\left|1+\frac{u}{\rho_{0}}\right| u^{k_{0}-1} d u,
$$

where $k_{0}$ is chosen so large that the integral converges. This is possible by 
(16.4). The dominant function is again independent of $z$ in $D$ and approaches zero with $1 / k$, so that (16.2) is completely established. In a precisely similar way (16.3) is established, an integration by parts being necessary since $|\phi(u)|$ need not be bounded in $(0, \delta)$. However, it is unnecessary to use (16.4) in the present case since $\alpha(u)$ is continuous and hence bounded in $0 \leqq u \leqq \delta$. We omit the details of the proof.

It remains to show that

$$
\lim _{k \rightarrow \infty} \frac{1}{k !}\left(\frac{k}{z}\right)^{k+1} \int_{\delta}^{\Delta} e^{-k u / z} u^{k} \phi(u) d u=\phi(z)
$$

uniformly in $D$. To establish this point we must alter the path of integration. We replace the segment of the real axis by a curve $C$ composed of three arcs

$C_{1}$ :

$$
\begin{aligned}
& r=\delta \\
& \theta=\psi \\
& r=\Delta
\end{aligned}
$$$$
(0 \leqq \theta \leqq \psi),
$$

$C_{2}$ :$$
(\delta \leqq r \leqq \Delta) \text {, }
$$

$C_{3}$ :$$
(0 \leqq \theta \leqq \psi) \text {. }
$$

We have here supposed that $\psi \geqq 0$. If $\psi<0$, replace the inequalities defining $C_{1}$ and $C_{3}$ by $\psi \leqq \theta \leqq 0$. Since the integrand of (16.6) is analytic in the region bounded by the line segment $\delta \leqq u \leqq \Delta$ and the curve $C$, we do not alter the value of the integral by changing the path of integration as indicated. It is to be noted that the curve $C$ changes as $z$ varies in $D$.

We now show that

$$
\lim _{k \rightarrow \infty} \frac{1}{k !}\left(\frac{k}{z}\right)^{k+1} \int_{C_{i}} e^{-\dot{k} u / z} u^{k} \phi(u) d u=0 \quad(i=1,3)
$$

uniformly in $D$. We prove it for $i=1$, the proof for $i=3$ being similar. We have

$$
\begin{aligned}
& \left|\frac{1}{k !}\left(\frac{k}{z}\right)^{k+1} \int_{C_{1}} e^{-k u / z} u^{k} \phi(u) d u\right| \\
& \quad \leqq \frac{1}{k !}\left(\frac{k}{\rho_{0}}\right)^{k+1} \int_{0}^{\psi_{1}} e^{-\left[k \delta \cos \psi_{1}\right] / \rho_{1} \delta^{k+1}}\left|\phi\left(\delta e^{i \theta}\right)\right| d \theta \\
& \quad \leqq \frac{k^{k+1}}{k !} \frac{\delta l^{k}}{\rho_{0}} \int_{0}^{\psi_{1}}\left|\phi\left(\delta e^{-i \theta}\right)\right| d \theta .
\end{aligned}
$$

This upper bound is independent of $z$ in $D$ and approaches zero with $1 / k$.

We have thus reduced our problem to that of showing that

$$
\lim _{k \rightarrow \infty} \frac{1}{k !}\left(\frac{k}{z}\right)^{k+1} \int_{\delta}^{\Delta} e^{-\left[k r e^{i \psi}\right] / z} r^{k} e^{i \psi(k+1)} \phi\left(r e^{i \psi}\right) d r=\phi(z)
$$


uniformly in $D$. But by Theorem 6

$$
\lim _{k \rightarrow \infty} \frac{k^{k+1}}{k !} \frac{1}{\rho^{k+1}} \int_{\delta}^{\Delta} e^{-k r / \rho_{r} k} d r=1
$$

uniformly* in the interval $\rho_{0} \leqq r \leqq \rho_{1}$. Hence we must show that

$$
\lim _{k \rightarrow \infty} \frac{k^{k+1}}{k !} \frac{1}{\rho^{k+1}} \int_{\delta}^{\Delta} e^{-k r / \rho_{r} k}\left[\phi\left(r e^{i \psi}\right)-\phi(z)\right] d r=0
$$

uniformly in $D$. Make the change of variable $v=r / \rho$. The integral becomes

$$
I(k)=\frac{k^{k+1}}{k !} \int_{\delta / \rho}^{\Delta / \rho} e^{-k v} v^{k}[\phi(v z)-\phi(z)] d v .
$$

Given an arbitrary positive $\epsilon$ we shall show that we can determine a number $k_{1}$ independent of $z$ in $D$ such that

$$
|I(k)|<\epsilon \quad\left(k>k_{1}\right) .
$$

We first observe that

$$
|I(k)| \leqq \frac{k^{k+1}}{k !} \int_{\delta / \rho_{1}}^{\Delta / \rho_{0}} e^{-k v_{v} k}|\phi(v z)-\phi(z)| d v .
$$

Since $\phi(z)$ is uniformly continuous in $z$ we can determine a number $\zeta$ such that

$$
\left|\phi\left(z^{\prime}\right)-\phi\left(z^{\prime \prime}\right)\right|<\epsilon / 3 \quad\left(\left|z^{\prime}-z^{\prime \prime}\right|<\zeta\right)
$$

provided only that $z^{\prime}$ and $z^{\prime \prime}$ are in $D$. Set $\eta=\zeta / \rho_{1}$. Then if $|1-v|<\eta$, we have

$$
|z-v z|<\rho_{1} \eta=\zeta \text {. }
$$

Hence if $z$ is in $D$ and $|1-v|<\eta$ it follows that

$$
|\phi(z)-\phi(v z)|<\epsilon / 3 \text {. }
$$

We now have

where

$$
|I(k)| \leqq I_{1}(k)+I_{2}(k)+I_{3}(k),
$$

$$
I_{1}(k)=\frac{k^{k+1}}{k !} \int_{\delta / \rho_{1}}^{1-\eta} e^{-k v} v^{k}|\phi(v z)-\phi(z)| d v,
$$

and where $I_{2}(k)$ and $I_{3}(k)$ are similar integrals with intervals of integration $(1-\eta, 1+\eta)$ and $\left(1+\eta, \Delta / \rho_{0}\right)$ respectively.

* Take the function $\phi(t)$ of Theorem 6 equal to zero for $0 \leqq t \leqq \delta$ and for $\Delta \leqq t<\infty$, and equal to unity for $\delta<t<\Delta$. Since $\delta<\rho_{0}<\rho_{1}<\Delta$, the interval $\left(\rho_{0}, \rho_{1}\right)$ is an interval of continuity of $\phi(t)$. 
By (16.7)

$$
\left|I_{2}(k)\right| \leqq \frac{k^{k+1}}{k !} \frac{\epsilon}{3} \int_{1-\eta}^{1+\eta} e^{-k v} v^{k} d v<\frac{\epsilon}{3} .
$$

If $z$ is in $D$, and if $\delta / \rho_{1} \leqq v \leqq \Delta / \rho_{0}$, then $v z$ surely lies in the closed region

$$
\rho_{0} \delta / \rho_{1} \leqq \rho \leqq \rho_{1} \Delta / \rho_{0}, \quad|\psi| \leqq \psi_{1} .
$$

Denote the maximum of $|\phi(z)|$ in that region by $N$. Then

$$
\begin{aligned}
& \left|I_{1}(k)\right| \leqq \frac{k^{k+1}}{k !} e^{-k(1-\eta)}(1-\eta)^{k} 2 N, \\
& \left|I_{3}(k)\right| \leqq \frac{k^{k+1}}{k !} e^{-k(1+\eta)}(1+\eta)^{k} 2 N .
\end{aligned}
$$

If we determine $k_{1}$ so large that the right-hand members of (16.8) and (16.9) are each less than $\epsilon / 3$ for $k>k_{1}$, we have

$$
|I(k)|<\epsilon
$$

This completes the proof of the theorem.

17. The zeros of the determining function. The applicability of our inversion formula in the complex domain enables us to extend the study of the zeros of the determining function made in Part III to the case when these zeros are no longer on the real axis. We first take the case in which the generating function is analytic at infinity and prove

THEOREM 33. Let the function $f(s)$ be analytic at infinity and have the representation

$$
f(s)=\int_{0}^{\infty} e^{-s z} \phi(z) d z .
$$

If $\phi(z)$ has $n$ zeros not at $z=0$ in the region $|z|<l$, then there exists an integer $k_{1}$ such that $f^{(k)}(s)$ has $n$ zeros not at $z=\infty$ in the region $|s|>k_{1} / l$ for $k \geqq k_{1}$.

For, suppose that in addition to the $n$ zeros of $\phi(z)$ which are not.at the origin there are $m$ zeros at the origin. Then $\phi(z)$ has $n+m$ zeros in the region $|z|<l$. By the Corollary of Theorem 31

$$
\lim _{k \rightarrow \infty} L_{k, z}[f(s)]=\lim _{k \rightarrow \infty} \frac{(-1)^{k}}{k !} f^{(k)}\left(\frac{k}{z}\right)\left(\frac{k}{z}\right)^{k+1}=\phi(z)
$$

uniformly in the region $|z| \leqq l$. Hence by a theorem of E. Rouché* there will exist an integer $k_{1}$ such that for $k \geqq k_{1}$ the function $L_{k, z}[f(s)]$, which is surely

* See for example Pólya and Szegö, loc. cit., vol. 1, p. 122, No. 194. 
analytic at $z=0$ if suitably defined there, will have exactly $n+m$ zeros in the region $|z|<l$. If $f(s)$ and $\phi(z)$ have the power series developments (15.2) and (15.3), then

$$
a_{0}=a_{1}=\cdots=a_{m-1}=0 .
$$

Consequently the function $f^{(k)}(s) s^{k+1}$ will have $m$ zeros at infinity and $L_{k, z}[f(s)]$ will have $m$ zeros at $z=0$ and hence $n$ zeros in the region $|z|<l$ not at $z=0$. But $f^{(k)}(k / z)$ has the same zeros as $L_{k, z}[f(s)]$ except at $z=0$. It follows that $f^{(k)}(s)$ has $n$ zeros not at $z=0$ in the region $|s|>k / l \geqq k_{1} / l$. This completes the proof of the theorem.

If $f(s)$ is no longer analytic at infinity we are not at liberty to suppose that $\phi(z)$ is entire. If $\phi(z)$ is analytic in the half-plane $x>0$ we may still obtain results concerning its zeros. We prove

TheORem 34. Let the function $\phi\left(\rho e^{i \psi}\right)$ be analytic in the half-plane $-\pi / 2$ $<\psi<\pi / 2$, and let

$$
f(s)=\int_{0}^{\infty} e^{-s \rho} \phi(\rho) d \rho,
$$

the integral converging for some value of $s$. If $\phi\left(\rho e^{i \psi}\right)$ has $n$ zeros in the region

$$
0<\rho_{1}<\rho<\rho_{2},|\psi|<\psi_{2}<\pi / 2,
$$

then for all $k$ sufficiently large $f^{(k)}(s)$ will have $n$ zeros in the region

$$
k / \rho_{2}<\rho<k / \rho_{1},|\psi|<\psi_{2} .
$$

By Theorem 32 we have

$$
\lim _{k \rightarrow \infty} L_{k, z}[f(s)]=\lim _{k \rightarrow \infty} \frac{(-1)^{k}}{k !} f^{(k)}\left(\frac{k}{z}\right)\left(\frac{k}{z}\right)^{k+1}=\phi(z)
$$

uniformly in the region

$$
\rho_{1} \leqq \rho \leqq \rho_{2},|\psi| \leqq \psi_{2} .
$$

By Rouchés Theorem there exists a number $k_{1}$ such that for $k \geqq k_{1}$ the function $L_{k, s}[f(s)]$ and hence also $f^{(k)}(k / z)$ has exactly $n$ zeros in the region

$$
\rho_{1}<\rho<\rho_{2},|\psi|<\psi_{2} \text {. }
$$

We obtain the result of the theorem by replacing $z$ by $k / s$.

We turn now to the complex analogue of Theorem 24 .

THEOREM 35. Let the function $\phi(z)$ be analytic in the half-plane $x>0$, and let

$$
f(s)=\int_{0}^{\infty} e^{-s z} \phi(z) d z,
$$


the integral converging for some value of $s$. If $\phi(z)$ has a zero at a point $z_{0}, x_{0}>0$, then for $k$ sufficiently large $f^{(k)}(s)$ will have a zero at a point $s_{k}$ such that

$$
\lim _{k \rightarrow \infty} \frac{s_{k}}{k}=\frac{1}{z_{0}} .
$$

Let the zero $z_{0}$ of $\phi(z)$ be of order $\lambda$. With $z_{0}$ as center describe a circle of radius $\eta$ so small that no other zero of $\phi(z)$ lies in it and such that the circle lies entirely in the half-plane $x>0$. Then by the Theorem of Rouche we can determine an integer $k_{1}$ so large that for $k>k_{1}$ the function $f^{(k)}(k / z)$ will have have exactly $\lambda$ zeros (distinct or coincident) inside this circle. Denote any one of them by $z_{k}$. Then $f^{(k)}(s)$ will vanish at the point $s_{k}=k / z_{k}$. Then

$$
\left|\frac{k}{s_{k}}-z_{0}\right|<\eta .
$$

Since $\eta$ was arbitrarily small it follows that

$$
\lim _{k \rightarrow \infty} \frac{k}{s_{k}}=z_{0} .
$$

In a similar way we could show that if $f(s)$ is analytic at infinity and if $\phi(z)$ vanishes at a point $z_{0}$ not the origin, there would exist a zero $s_{k}$ of $f^{(k)}(s)$ for $k$ sufficiently large such that $k / s_{k}$ approaches $z_{0}$ as $k$ becomes infinite.

We illustrate the result by several examples. The first example following Theorem 24 gives us an interesting example of the present theory in case $a=b>0$. Then $\phi(t)$ has a zero at $a$ but no change of sign there, so that the real theory fails. We find that $f^{(k)}(s)$ has the complex zeros

$$
\begin{aligned}
& s_{k, 1}=\left[(k+1)+i(k+1)^{1 / 2}\right] / a, \\
& s_{k, 2}=\left[(k+1)-i(k+1)^{1 / 2}\right] / a .
\end{aligned}
$$

Hence $f^{(k)}(s)$ will have no real zeros however large $k$ may be. But it will have two complex zeros for all $k$ and

$$
\lim _{k \rightarrow \infty} \frac{s_{k, j}}{k}=\frac{1}{a}
$$$$
(j=1,2) \text {. }
$$

The same analysis holds if $a<0$ or if $a$ is complex, and provides an example to illustrate the case in which the zeros of $\phi(z)$ may be in the half-plane $x<0$. Of course $f(s)$ is analytic at infinity. 


\section{PART V}

\section{THE MOMENT PROBLEM}

18. The problem. In this part we shall consider the infinite system of equations

$$
\mu_{n}=\int_{0}^{1} t^{n} d \alpha(t) \quad(n=0,1,2, \cdots),
$$

where $\alpha(t)$ is of bounded variation in the interval $(0,1)$ and $\alpha(1)=0$. The relation of this system of equations to the integral equation (1.1) becomes evident if the substitution $t=e^{-u}$ is made:

$$
\mu_{n}=\int_{0}^{\infty} e^{-n u} d\left\{-\alpha\left(e^{-u}\right)\right\} \quad(n=0,1,2, \cdots) .
$$

Here the variable $n$, running through a discrete set, replaces the continuous variable $x$ of (1.1). We should expect then to be able to determine the function $\alpha(t)$ in terms of the sequence $\left\{\mu_{n}\right\}$ by use of an operator similar to (3.1), (3.2), replacing the derivatives of $f(x)$ in that expression by the differences of $\left\{\mu_{n}\right\}$ and the integral sign by a summation sign. We shall find that the expected analogy is complete and that we can also obtain in a similar way an operator analogous to $L_{t}[f(x)]$ which, when applied to a sequence

will yield the function $\phi(t)$.

$$
\mu_{n}=\int_{0}^{1} t^{n} \phi(t) d t
$$

19. An extension of the Laplace method. In the following section we shall need an extension of the classical Laplace method for the asymptotic evaluation of a definite integral. We state the result that we shall need as a

Lemma. Let the function $h(x)$ be of class $C^{\prime \prime}$ in the interval $a \leqq x \leqq b$ and satisfy the conditions

$$
h^{\prime}(b)=0, h^{\prime \prime}(b)<0, h(x)<h(b) \quad(a \leqq x<b) ;
$$

let the functions $\phi_{1}(x), \phi_{2}(x), \cdots$ be of class $C^{\prime}$ in $a \leqq x \leqq b$ and satisfy the conditions

$$
\begin{aligned}
\left|\phi_{k}(x)\right| \leqq \psi(x) & (a \leqq x \leqq b), \\
\left|\phi_{k}{ }^{\prime}(x)\right| \leqq M & (a \leqq x \leqq b ; k=1,2, \cdots), \\
\left|\phi_{k}(b)\right| & \geqq 1
\end{aligned}
$$

where $\psi(x)$ is integrable in $(a, b)$ and $M$ is a constant independent of $k$. Then

$$
\int_{a}^{b} \phi_{k}(x) e^{k h(x)} d x \sim \phi_{k}(b) e^{k h(b)}\left(\frac{-\pi}{2 k h^{\prime \prime}(b)}\right)^{1 / 2}
$$


This reduces to the classical result if the functions $\phi_{k}(x)$ are all equal to a single function $\psi(x)$. Since the proof is much the same as in the classical case we omit it here.*

20. A preliminary limit. We begin with a consideration of the limit of

$$
H_{k}(t)=\sum_{i=n+1}^{\infty} \frac{(i+k) !}{i ! k !} t_{0}{ }^{i}\left(1-t_{0}\right)^{k+1} \quad\left(n=\left[\frac{k t}{1-t}\right], 0<t_{0}<1\right)
$$

as $k$ becomes infinite. Here, as in the remainder of the paper, the notation $[u]$ means the largest integer contained in $u$. Taylor's series with exact remainder for the function $(1-x)^{-k-1}$ gives

$$
\left(1-t_{0}\right)^{-k-1}=\sum_{i=0}^{n} \frac{(k+i) !}{k ! i !} t_{0}^{i}+\frac{(n+k+1) !}{n ! k !} \int_{0}^{t_{0}} \frac{\left(t_{0}-x\right)}{(1-x)^{n+k+2}} d x .
$$

Hence

$$
H_{k}(t)=\left(1-t_{0}\right)^{k+1} \frac{(n+k+1) !}{n ! k !} \int_{0}^{t_{0}} \frac{\left(t_{0}-x\right)^{n}}{(1-x)^{n+k+2}} d x,
$$

or, if we set $u=\left(t_{0}-x\right) /(1-x)$,

$$
H_{k}(t)=\frac{(n+k+1) !}{n ! k !} \int_{0}^{t_{0}} u^{n}(1-u)^{k} d u .
$$

Let us first consider $H_{k}\left(t_{0}\right)$. Set

$$
v_{0}=t_{0} /\left(1-t_{0}\right), \alpha=k v_{0}-n .
$$

Then $\alpha$ depends on $k$ and satisfies the relation

$$
0 \leqq \alpha<1 \text {. }
$$

Since

we have

$$
\int_{0}^{1} u^{n}(1-u)^{k} d u=\frac{n ! k !}{(n+k+1) !}
$$

$$
\frac{1}{H_{k}\left(t_{0}\right)}-1=\frac{\int_{t_{0}}^{1}\left\{u^{v_{0}}(1-u)\right\}^{k} u^{-\alpha} d u}{\int_{0}^{t_{0}}\left\{u^{v_{0}}(1-u)\right\}^{k} u^{-\alpha} d u} .
$$

By use of the Lemma of the previous section we shall show that this quantity approaches unity and hence that $H_{k}\left(t_{0}\right)$ approaches $1 / 2$ as $k$ becomes infinite. We first obtain an asymptotic expression for the integral

* See, for example, P6lya and Szegö, Aufgaben und Lehrsätze aus der Analysis, vol. I, p. 80 , problem 212 . 


$$
\int_{0}^{t_{0}}\left\{u^{v_{0}}(1-u)\right\}^{k} u^{-\alpha} d u
$$

Let $\delta$ be any positive quantity less than $t_{0}$. Then

$$
\begin{gathered}
\int_{0}^{\delta}\left\{u^{v_{0}}(1-u)\right\}^{k} u^{-\alpha} d u<\left\{\delta^{v_{0}}(1-\delta)\right\}^{k-1} \int_{0}^{\delta} u^{v_{0}-1}(1-u) d u \\
=\left\{t_{0}^{v_{0}}\left(1-t_{0}\right)\right\}^{k} O\left(\beta^{k}\right)
\end{gathered}
$$

where $\beta$ is a positive constant less than unity and independent of $k$. We now apply the Lemma to the integral

$$
\int_{\delta}^{t_{0}}\left\{u^{v_{0}}(1-u)\right\}^{k} u^{-\alpha} d u \text {. }
$$

For the application we have

$$
\begin{gathered}
h(x)=v_{0} \log x+\log (1-x), \\
h^{\prime}(x)>0\left(x<t_{0}\right), \\
h^{\prime}\left(t_{0}\right)=0, \quad h^{\prime \prime}\left(t_{0}\right)=-\frac{1}{t_{0}\left(1-t_{0}\right)^{2}}<0, \\
\phi_{k}(x)=x^{-\alpha}, \quad\left|\phi_{k}^{\prime}(x)\right|<1 / \delta^{2}=M, \\
\phi_{k}\left(t_{0}\right)=t_{0}^{-\alpha}>1 .
\end{gathered}
$$

Hence we conclude that

$$
\int_{\delta_{0}}^{t_{0}}\left\{u^{v_{0}}(1-u)\right\}^{k} u^{-\alpha} d u \sim t_{0}{ }^{n+(1 / 2)}\left(1-t_{0}\right)^{k+1}(\pi /(2 k))^{1 / 2} \quad(k \rightarrow \infty) .
$$

By virtue of (20.3) we see that the integral (20.2) has this same asymptotic expression. Similar reasoning shows that

$$
\int_{t_{0}}^{1}\left\{u^{v_{0}}(1-u)\right\}^{k} u^{-\alpha} d u \sim t_{0}^{n+(1 / 2)}\left(1-t_{0}\right)^{k+1}(\pi /(2 k))^{1 / 2} \quad(k \rightarrow \infty)
$$

so that

$$
\lim _{k \rightarrow \infty} H_{k}\left(t_{0}\right)=\frac{1}{2}
$$

Next consider the case $t>t_{0}$. Set

$$
v=t /(1-t) .
$$

Since the function $u^{v}(1-u)$ is increasing in the interval $0 \leqq u \leqq t_{0}$ (with its maximum at $\left.u=t>t_{0}\right)$, it follows that 


$$
\begin{gathered}
\int_{0}^{t_{0}} u^{n}(1-u)^{k} d u<\left\{t_{0} v\left(1-t_{0}\right)\right\}^{k-1}, \\
H_{k}(t)<\frac{\Gamma(v k+k+2)}{\Gamma(k+1) \Gamma(k v+1)}\left\{t_{0}^{v}\left(1-t_{0}\right)\right\}^{k-1} .
\end{gathered}
$$

By use of Stirling's formula we can show that the right-hand side of this inequality approaches zero with $1 / k$. Thus

$\lim _{k \rightarrow \infty} H_{k}(t)=\lim _{k \rightarrow \infty}\left(\frac{v k+k+1}{e}\right)^{v k+k+1}\left(\frac{e}{k}\right)^{k}\left(\frac{e}{v k}\right)^{v k}\left\{t_{0^{0}}\left(1-t_{0}\right)\right\}^{k-1}\left(\frac{v+1}{2 k \pi v}\right)^{1 / 2}$.

The function of $k$ on the right may be regarded as the $k$ th term of an infinite series whose test ratio is

$$
\left(\frac{v+1}{v}\right)^{v}(v+1) t_{0}\left(1-t_{0}\right)
$$

We can show that this is less than unity. Introducing $t$ and setting $t-t_{0}=\eta$ we must show that

$$
\left(1-\frac{\eta}{t}\right)^{t /(1-t)}\left(1+\frac{\eta}{1-t}\right)<1 .
$$

Employing a familiar inequality we have

$$
\begin{aligned}
& \left(1-\frac{\eta}{t}\right)^{t}<1-\eta, \\
& \left(1+\frac{\eta}{1-t}\right)^{1-t}<1+\eta, \\
& \left(1-\frac{\eta}{t}\right)^{t}\left(1+\frac{\eta}{1-t}\right)^{1-t}<1-\eta^{2}<1,
\end{aligned}
$$

from which (20.4) follows at once. Hence the series in question converges and

$$
\lim _{k \rightarrow \infty} H_{k}(t)=0 \quad\left(t>t_{0}\right) .
$$

Finally, if $0<t<t_{0}$, we write

$$
\begin{aligned}
1-H_{k}(t) & =\frac{(n+k+1) !}{n ! k !} \int_{t_{0}}^{1} u^{n}(1-u)^{k} d u \\
1-H_{k}(t) & <\frac{\Gamma(v k+k+2)}{\Gamma(k+1) \Gamma(v k+1)}\left\{t_{0} v\left(1-t_{0}\right)\right\}^{k} \frac{1}{t_{0}} .
\end{aligned}
$$

We treat the right-hand side of this inequality as before. We are again led to 
prove (20.4), but now $\eta$ is a negative quantity greater than -1 . The inequalities employed are not affected by this change so that

$$
\lim _{k \rightarrow \infty}\left[1-H_{k}(t)\right]=0 .
$$

Consequently we have proved

THEOREM 36. If

then

$$
\begin{aligned}
H_{k}(t) & =\sum_{i=n+1}^{\infty} \frac{(i+k) !}{i ! k !} t_{0}{ }^{i}\left(1-t_{0}\right)^{k+1} \quad\left(n=\left[\frac{k t}{1-t}\right]\right), \\
& =\frac{(n+k+1) !}{n ! k !} \int_{0}^{t_{0}} u^{n}(1-u)^{k} d u,
\end{aligned}
$$

$$
\lim _{k \rightarrow \infty} H_{k}(t)=\left\{\begin{array}{lr}
1 & \left(0<t<t_{0}\right), \\
\frac{1}{2} & \left(t=t_{0}\right), \\
0 & \left(t_{0}<t<1\right) .
\end{array}\right.
$$

We have really solved here the system of moment equations

and found that

$$
\mu_{n}=\frac{t_{0}^{n+1}}{n+1}=\int_{0}^{1} t^{n} \phi(t) d t
$$

$$
\phi(t)=\lim _{k \rightarrow \infty} \frac{(n+k+1) !}{n ! k !}(-1)^{k} \Delta^{k} \mu_{n}, \quad n=\left[\frac{k t}{1-t}\right] .
$$

21. Inversion of the general sequence of moments. We now introduce the following operators.

Definition. An operator $S_{t}\left\{\mu_{n}\right\}$ is defined by the equations

$$
\begin{aligned}
S_{k, \ell}\left\{\mu_{n}\right\} & =-\mu_{\infty}-\sum_{i=n+1}^{\infty} \frac{(i+k) !}{i ! k !}(-1)^{k+1} \Delta^{k+1} \mu_{i}, \quad n=\left[\frac{k t}{1-t}\right], \\
S_{t}\left\{\mu_{n}\right\} & =\lim _{k \rightarrow \infty} S_{k, \ell}\left\{\mu_{n}\right\} .
\end{aligned}
$$

Definition. An operator $L_{t}\left\{\mu_{n}\right\}$ is defined by the equations

$$
\begin{aligned}
L_{k, t}\left\{\mu_{n}\right\} & =\frac{(n+k+1) !}{n ! k !}(-1)^{k} \Delta^{k} \mu_{n}, \quad n=\left[\frac{k t}{1-t}\right], \\
L_{t}\left\{\mu_{n}\right\} & =\lim _{k \rightarrow \infty} L_{k, t}\left\{\mu_{n}\right\} .
\end{aligned}
$$

We shall now show that $S_{t}\left\{\mu_{n}\right\}$ inverts the sequence (18.1). 
THEOREM 37. If the function $\alpha(t)$ is of bounded variation in the interval $(0,1)$ with $\alpha(1)=0$, and if

then

$$
\mu_{n}=\int_{0}^{1} t^{n} d \alpha(t) \quad(n=0,1,2, \cdots),
$$

$$
S_{t}\left\{\mu_{n}\right\}=\frac{\alpha(t+)+\alpha(t-)}{2} \quad(0<t<1) .
$$

An integration by parts gives us

Then

$$
\mu_{n}=-n \int_{0}^{1} t^{n-1} \alpha(t) d t \quad(n=1,2, \cdots) .
$$

$$
\nu_{n}=-\frac{\mu_{n+1}}{n+1}=\int_{0}^{1} t^{n} \alpha(t) d t \quad(n=0,1,2, \cdots) .
$$

We first show that $L_{t}\left\{\nu_{n}\right\}$ is well defined. We have

$$
L_{k, t}\left\{\nu_{n}\right\}=\frac{(n+k+1) !}{n ! k !} \int_{0}^{1} t^{n}(1-t)^{k} \alpha(t) d t, \quad n=\left[\frac{k t}{1-t}\right] .
$$

Let $t_{0}$ be an arbitrary point in the interval $0<t<1$. Set

Form the function

$$
g(t)=\left\{\begin{array}{rr}
-1 & \left(0<t<t_{0}\right) \\
-\frac{1}{2} & \left(t=t_{0}\right), \\
0 & \left(t_{0}<t<1\right) .
\end{array}\right.
$$

$$
\psi(t)=g(t)\left\{\alpha\left(t_{0}+\right)-\alpha\left(t_{0}-\right)\right\}+\alpha\left(t_{0}+\right),
$$

so that the function

$$
\phi(u)=\alpha(u)-\psi(u)
$$

has the property that

$$
\phi\left(t_{0}+\right)=\phi\left(t_{0}-\right)=0 .
$$

Now set up the integral

$$
I=\frac{(n+k+1) !}{n ! k !} \int_{0}^{1} y^{n}(1-y)^{k} \phi(y) d y \quad\left(n=\left[\frac{k t_{0}}{1-t_{0}}\right]\right) .
$$

We divide the interval of integration into three parts $(0,1-\eta),(1-\eta, 1+\eta)$, $(1+\eta, 1)$, denoting the corresponding integrals by $I_{1}, I_{2}, I_{3}$ respectively. Given an arbitrary positive $\epsilon$ we determine $\eta$ so small that

$$
|\phi(y)|=|\alpha(y)-\psi(y)|<\epsilon / 3 \quad\left(0<\left|y-t_{0}\right| \leqq \eta\right) .
$$


Then we have

$$
\begin{aligned}
& \left|I_{2}\right| \leqq \frac{(n+k+1) !}{n ! k !} \int_{0}^{1} y^{n}(1-y)^{k} \frac{\epsilon}{3} d y=\frac{\epsilon}{3}, \\
& \left|I_{2}\right| \leqq \frac{(n+k+1) !}{n ! k !}\left(t_{0}-\eta\right)^{(k-1) t_{0} /\left(1-t_{0}\right)}\left(1-t_{0}+\eta\right)^{k-1} M, \\
& \left|I_{3}\right| \leqq \frac{(n+k+1) !}{n ! k !}\left(t_{0}+\eta\right)^{(k-1) t_{0} /\left(1-t_{0}\right)}\left(1-t_{0}-\eta\right)^{k-1} M,
\end{aligned}
$$

where $M$ is a suitably chosen constant. We have already seen in $\$ 20$ that the right-hand members of the last two inequalities approach zero with $1 / k$, so that it is clearly possible to determine $k_{0}$ so large that

for $k>k_{0}$. But

$$
|I|<\epsilon
$$

$$
\begin{aligned}
I= & L_{k, t}\left\{\nu_{n}\right\} \\
& +\left\{\alpha\left(t_{0}+\right)-\alpha\left(t_{0}-\right)\right\} \frac{(n+k+1) !}{n ! k !} \int_{0}^{t_{0}} y^{n}(1-y)^{k} d y-\alpha\left(t_{0}+\right),
\end{aligned}
$$

so that by allowing $k$ to become infinite we obtain

$$
\begin{gathered}
0=L_{t}\left\{\nu_{n}\right\}+\frac{\alpha\left(t_{0}+\right)-\alpha\left(t_{0}-\right)}{2}-\alpha\left(t_{0}+\right), \\
L_{t}\left\{\nu_{n}\right\}=\frac{\alpha\left(t_{0}+\right)+\alpha\left(t_{0}-\right)}{2} .
\end{gathered}
$$

To evaluate the limit of the second term on the right-hand side of (21.1) we have employed Theorem 36.

It remains to show that

$$
L_{k, t}\left\{\nu_{n}\right\}=S_{*, t}\left\{\mu_{n}\right\} .
$$

To do this we prove first that

$$
\lim _{i \rightarrow \infty} \frac{(i+k+1) !}{(k+1) ! i !} \Delta^{k+1} \mu_{i+1}=\lim _{i \rightarrow \infty} \frac{(i+k+1) !}{(k+1) ! i !} \int_{0}^{1} t^{i+1}(1-t)^{k+1} d \alpha(t)=0
$$

and that $\mu_{\infty}$ exists. Introduce the function

Then

$$
\omega(t)=\alpha(t)-\alpha(1-) \quad(0 \leqq t \leqq 1) .
$$

$$
I_{i, k}=\int_{0}^{1} t^{i+1}(1-t)^{k} d \alpha(t)=\int_{0}^{1} t^{i+1}(1-t)^{k} d \omega(t) \quad(k=0,1,2, \cdots) .
$$


In particular

$$
\begin{aligned}
\mu_{i+1} & =\int_{0}^{1} t^{i+1} d \alpha(t)=\int_{0}^{1} t^{i+1} d \omega(t) \\
& =-\alpha(1-)-(i+1) \int_{0}^{1} t^{i} \omega(t) d t(i=0,1,2, \cdots) .
\end{aligned}
$$

Given an arbitrary positive $\epsilon$, we determine a number $\delta$ such that

Then

$$
|\omega(t)|<\epsilon / 2 \quad(1-\delta \leqq t<1) .
$$

$$
\begin{aligned}
\left|(i+1) \int_{0}^{1} t^{i} \omega(t) d t\right| & \leqq\left|(i+1) \int_{0}^{1-\delta} t^{i} \omega(t) d t\right|+(i+1) \int_{1-\delta}^{1} t^{i} \frac{\epsilon}{2} d t \\
& \leqq(1-\delta)^{i+1}+\epsilon / 2,
\end{aligned}
$$

where $N$ is an upper bound for $|\omega(t)|$ in $(0,1)$. We can now determine $i$ so large that $(1-\delta)^{i+1} N$ is less than $\epsilon / 2$, so that

Next consider

$$
\mu_{\alpha}=-\alpha(1-) \text {. }
$$

$$
I_{i, k}=\int_{0}^{1} t^{i+1}(1-t)^{k} d \omega(t)=\int_{0}^{1} t^{i+1} d \beta(t)=-(i+1) \int_{0}^{1} t^{i} \beta(t) d t,
$$

where

$$
\begin{aligned}
& \beta(t)=\int_{t}^{1}(1-x)^{k} d \omega(x) \\
& (0 \leqq t<1), \\
& \beta(1)=0 \text {. }
\end{aligned}
$$

Since $\beta(t)$ is continuous at $t=1$, it follows that

$$
\beta(t)=o\left((1-t)^{k}\right)
$$

Hence it is easily seen that

$$
I_{i, k}=o\left\{(i+1) \int_{0}^{1} t^{i}(1-t)^{k} d t\right\}=o\left\{\frac{(i+1) k !}{(i+k+1) !}\right\} \quad(i \rightarrow \infty) .
$$

Consequently we have proved that

$$
\lim _{i \rightarrow \infty} \frac{(i+k+1) !}{(k+1) ! i !}(-1)^{k+1} \Delta^{k+1} \mu_{i+1}=0 \quad(k=0,1,2, \cdots) .
$$

With this fact at our disposal we shall be able to show that the series

$$
\mu_{\infty}+\sum_{i=n}^{\infty} \frac{(i+k+1) !}{k !(i+1) !}(-1)^{k+1} \Delta^{k+1} \mu_{i+1}
$$


converges and has the sum

$$
\sum_{i=0}^{k} \frac{(n+i) !}{i ! n !}(-1)^{i} \Delta^{i} \mu_{n+1} .
$$

We proceed by induction. For $k=0$ the relation reduces to

$$
\mu_{\infty}-\sum_{i=n}^{\infty} \Delta \mu_{i+1}=\mu_{n+1}
$$

The series converges since $\mu_{i}$ is known to approach a limit as $i$ becomes infinite, and partial summation shows the equation to be true. Now assume that (21.3) is equal to (21.4) and prove that the same equation holds when $k$ is replaced by $k+1$. Then

$$
\begin{aligned}
\sum_{i=0}^{k+1} \frac{(n+i) !}{n ! i !}(-1)^{i} \Delta^{i} \mu_{n+1}=\mu_{\infty} & +\sum_{i=n}^{\infty} \frac{(i+k+1) !}{k !(i+1) !}(-1)^{k+1} \Delta^{k+1} \mu_{i+1} \\
& +\frac{(n+k+1) !}{(k+1) ! n !}(-1)^{k+1} \Delta^{k+1} \mu_{n+1}
\end{aligned}
$$

We observe that

$$
\frac{(i+k+2) !}{(k+1) !(i+1) !}-\frac{(i+k+1) !}{(k+1) ! i !}=\frac{(i+k+1) !}{k !(i+1) !}
$$

and apply partial summation to the right-hand side of (21.5). By virtue of (21.2) we thus obtain

$$
\sum_{i=0}^{k+1} \frac{(n+i) !}{n ! i !}(-1)^{i} \Delta^{i} \mu_{n+1}=\mu_{\infty}+(-1)^{k+2} \sum_{i=n}^{\infty} \frac{(i+k+2) !}{(k+1) !(i+1) !} \Delta^{k+2} \mu_{i+1} .
$$

The induction is complete.

But (21.3) is $-S_{k, t}\left\{\mu_{n}\right\}$. Also (21.4) is $-L_{k, t}\left\{\nu_{n}\right\}$. For,

$$
\begin{aligned}
\Delta^{k}\left\{\frac{\mu_{n+1}}{n+1}\right\}= & \sum_{i=0}^{k}\left(\begin{array}{c}
k \\
i
\end{array}\right) \Delta^{i} \mu_{n+1} \Delta^{k-i}\left\{\frac{1}{n+i+1}\right\} \\
= & \sum_{i=0}^{k}(-1)^{k-i}\left(\begin{array}{c}
k \\
i
\end{array}\right) \Delta^{i} \mu_{n+1} \frac{(n+i) !(k-i) !}{(n+k+1) !} \\
& -\frac{(n+k+1) !}{n ! k !}(-1)^{k} \Delta^{k} \nu_{n} \\
= & \sum_{i=0}^{k} \frac{(n+i) !}{i ! n !}(-1)^{i} \Delta^{i} \mu_{n+1}=-L_{k, t}\left\{\nu_{n}\right\} .
\end{aligned}
$$

Hence 


$$
S_{t}\left\{\mu_{n}\right\}=\lim _{k \rightarrow \infty} S_{k, t}\left\{\mu_{n}\right\}=\lim _{k \rightarrow \infty} L_{k, t}\left\{\nu_{n}\right\}=\frac{\alpha(t+)+\alpha(t-)}{2},
$$

and the proof of the theorem is complete.

In the course of the proof we have demonstrated

THEOREM 38. If the function $\phi(t)$ is of bounded variation in the interval $(0,1)$ with $\phi(1)=0$ and if

then

$$
\mu_{n}=\int_{0}^{1} t^{n} \phi(t) d t \quad(n=0,1,2, \cdots)
$$

$$
L_{t}\left\{\mu_{n}\right\}=\frac{\phi(t+)+\phi(t-)}{2} \quad(0<t<1) .
$$

We next establish the stronger result contained in

THEOREM 39. If the function $\phi(t)$ is integrable in $(0,1)$ and if

then

$$
\mu_{n}=\int_{0}^{1} t^{n} \phi(t) d t \quad(n=0,1,2, \cdots),
$$

$$
L_{t}\left\{\mu_{n}\right\}=\phi(t)
$$

almost everywhere in $(0,1)$.

As we observed in $\$ 4$

$$
\int_{t}^{x}|\phi(u)-\phi(t)| d u=o(|x-t|)
$$

for almost all values of $t$ in $(0,1)$. Let $t_{0}$ be such a value of $t$, and form the integrals

$$
\begin{aligned}
& I_{k}=\frac{(n+k+1) !}{n ! k !} \int_{0}^{t_{0}} y^{n}(1-y)^{k}\left\{\phi(y)-\phi\left(t_{0}\right)\right\} d y, \\
& J_{k}=\frac{(n+k+1) !}{n ! k !} \int_{t_{0}}^{1} y^{n}(1-y)^{k}\left\{\phi(y)-\phi\left(t_{0}\right)\right\} d y, \quad n=\left[\frac{k t_{0}}{1-t_{0}}\right] .
\end{aligned}
$$

It will be sufficient to show that $I_{k}$ and $J_{k}$ approach zero with $1 / k$; for,

$$
\begin{aligned}
L_{k, t_{0}}\left\{\mu_{n}\right\} & =\frac{(n+k+1) !}{n ! k !} \int_{0}^{1} y^{n}(1-y)^{k} \phi(y) d y, \\
L_{t_{0}}\left\{\mu_{n}\right\} & =\phi\left(t_{0}\right)+\lim _{k \rightarrow \infty}\left\{I_{k}+J_{k}\right\} .
\end{aligned}
$$

Since the proof is similar for the two integrals we give only that for $I_{k}$. Set 


$$
\gamma(t)=\int_{t_{0}}^{t} \frac{\left|\phi(y)-\phi\left(t_{0}\right)\right|}{y} d y .
$$

It is easy to see by integration by parts that $\gamma(t)$ is also $o\left(t_{0}-t\right)$ as $t$ approaches $t_{0}$. Hence to an arbitrary positive $\epsilon$ there corresponds a number $\delta$ such that

$$
|\gamma(y)|<\left(t_{0}-y\right) \epsilon / 2 \quad\left(0<t_{0}-\delta<y<t_{0}\right) .
$$

Introduce the integrals

so that

$$
\begin{aligned}
& I_{k}^{\prime}=\frac{(n+k+1) !}{n ! k !} \int_{t_{0}-\delta}^{t_{0}} y^{n}(1-y)^{k}\left\{\phi(y)-\phi\left(t_{0}\right)\right\} d y, \\
& I_{k}^{\prime \prime}=\frac{(n+k+1) !}{n ! k !} \int_{0}^{t_{0}-\delta} y^{n}(1-y)^{k}\left\{\phi(y)-\phi\left(t_{0}\right)\right\} d y,
\end{aligned}
$$

If we write

we have

$$
I_{k}=I_{k}^{\prime}+I_{k}^{\prime \prime} \text {. }
$$

$$
\alpha=\frac{k t_{0}}{1-t_{0}}-\left[\frac{k t_{0}}{1-t_{0}}\right]
$$

$$
\begin{aligned}
I_{k}^{\prime}=\frac{(n+k+1) !}{n ! k !} \int_{t_{0}-\delta}^{t_{0}} y^{k t_{0} /\left(1-t_{0}\right)}(1-y)^{k} y^{-\alpha}\left\{\phi(y)-\phi\left(t_{0}\right)\right\} d y, \\
\begin{aligned}
\left|\frac{n ! k !}{(n+k+1) !} I_{k}^{\prime}\right| \leqq & \int_{t_{0}-\delta}^{t_{0}} y^{k t_{0} /\left(1-t_{0}\right)}(1-y)^{k} y^{-1}\left|\phi(y)-\phi\left(t_{0}\right)\right| d y \\
= & -\gamma\left(t_{0}-\delta\right)\left(t_{0}-\delta\right)^{k t_{0} /\left(1-t_{0}\right)}\left(1-t_{0}+\delta\right)^{k} \\
& -\int_{t_{0}-\delta}^{t_{0}} \gamma(y) d\left\{y^{k t_{0} /\left(1-t_{0}\right)}(1-y)^{k}\right\} .
\end{aligned}
\end{aligned}
$$

Noting that the function

$$
y^{k t_{0} /\left(1-t_{0}\right)}(1-y)^{k}
$$

is increasing in the interval $\left(t_{0}-\delta, t_{0}\right)$ and applying (21.6) we obtain

$$
\begin{aligned}
\frac{n ! k !}{(n+k+1) !}\left|I_{k}^{\prime}\right| & <\frac{\epsilon}{2} \delta\left(t_{0}-\delta\right)^{k t_{0} /\left(1-t_{0}\right)}\left(1-t_{0}+\delta\right)^{k} \\
& +\frac{\epsilon}{2} \int_{t_{0}-\delta}^{t_{0}}\left(t_{0}-y\right) d\left\{y^{k t_{0} /\left(1-t_{0}\right)}(1-y)^{k}\right\} \\
= & \frac{\epsilon}{2} \int_{t_{0}-\delta}^{t_{0}} y^{k t_{0} /\left(1-t_{0}\right)}(1-y)^{k} d y<\frac{\epsilon}{2} \int_{0}^{1} y^{n}(1-y)^{k} d y \\
& \left|I_{k}^{\prime}\right|<\epsilon / 2 .
\end{aligned}
$$


We turn next to $I_{k}^{\prime \prime}$. It may evidently be written as

$$
I_{k}^{\prime \prime}=\frac{(n+k+1) !}{n ! k !} \int_{0}^{t_{0}-\delta} y^{k t_{0} /\left(1-t_{0}\right)}(1-y)^{k} y^{-\alpha}\left\{\phi(y)-\phi\left(t_{0}\right)\right\} d y .
$$

Let $k_{0}$ be an integer so large that

Then

$$
\frac{k_{0} t_{0}}{1-t_{0}}>1
$$

$$
y^{k_{0} t_{0} /\left(1-t_{0}\right)} y^{-\alpha}(1-y)^{k_{0}}<1 \quad(0 \leqq y \leqq 1),
$$

and

$$
\frac{n ! k !}{(n+k+1) !}\left|I_{k}^{\prime \prime}\right| \leqq \int_{0}^{t_{0}-\delta} y^{\left(k-k_{0}\right) t_{0} /\left(1-t_{0}\right)}(1-y)^{k-k_{0}}\left|\phi(y)-\phi\left(t_{0}\right)\right| d y .
$$

The function

$$
y^{\left(k-k_{0}\right) t_{0} /\left(1-t_{0}\right)}(1-y)^{k-k_{0}}
$$

is increasing in the interval $\left(0, t_{0}\right)$, so that

$$
\left|I_{k}^{\prime \prime}\right| \leqq \frac{(n+k+1) !}{n ! k !}\left(t_{0}-\delta\right)^{\left(k-k_{0}\right) t_{0} /\left(1-t_{0}\right)}\left(1-t_{0}+\delta\right)^{k-k_{0} M},
$$

where

$$
M=\int_{0}^{t_{0}-\delta}\left|\phi(y)-\phi\left(t_{0}\right)\right| d y .
$$

But the right-hand side of this inequality tends to zero with $1 / k$, as we proved in $\$ 20$, so that we can determine $k_{1}$ so large that

and

$$
\left|I_{k}^{\prime \prime}\right|<\epsilon / 2
$$

$$
\left|I_{k}\right|<\epsilon \text {. }
$$

The theorem is thus established.

As illustrations of Theorems 39 and 41 it is interesting to show by direct evaluation of the limits concerned that

$$
\begin{array}{ll}
L_{t}\left\{\frac{1}{n+2}\right\}=t & (0<t<1) \\
S_{t}\left\{\frac{1}{n+2}\right\}=\frac{t^{2}-1}{2} & (0<t<1) .
\end{array}
$$

22. Uniqueness theorems. We are now in a position to establish 
THEOREM 40. If the sequence $\left\{\mu_{n}\right\}$ satisfies the inequalities

$$
\left|\Delta^{k} \mu_{n}\right|<\frac{M n ! k !}{(n+k+1) !} \quad(n=0,1,2, \cdots ; k=0,1,2, \cdots)
$$

then

$$
\mu_{m}=\lim _{k \rightarrow \infty} \int_{0}^{1} t^{m} L_{k, t}\left\{\mu_{n}\right\} d t \quad(m=0,1,2, \cdots) .
$$

By definition of the operator $L_{k, t}\left\{\mu_{n}\right\}$ we have

$$
I_{k}(m)=\int_{0}^{1} t^{m} L_{k, t}\left\{\mu_{n}\right\} d t=(-1)^{k} \int_{0}^{1} t^{m} \frac{(n+k+1) !}{n ! k !} \Delta^{k} \mu_{n} d t
$$

where

$$
n=\left[\frac{k t}{1-t}\right]
$$

Set

$$
\begin{aligned}
& H_{k}(m)=(-1)^{k} \int_{m /(k+m)}^{1} t^{m} \frac{(n+k+1) !}{n ! k !} \Delta^{k} \mu_{n} d t \\
& J_{k}(m)=(-1)^{k} \int_{0}^{m /(k+m)} t^{m} \frac{(n+k+1) !}{n ! k !} \Delta^{k} \mu_{n} d t
\end{aligned}
$$

so that

$$
I_{k}(m)=H_{k}(m)+J_{k}(m) .
$$

Our theorem will then be established if we can show that

$$
\lim _{k \rightarrow \infty} H_{k}(m)=\mu_{m}, \quad \lim _{k \rightarrow \infty} J_{k}(m)=0 \quad(m=0,1,2, \cdots) .
$$

In the integral (22.2) make the change of variable

$$
u=k t /(1-t)
$$

so that

$(22.3) H_{k}(m)=(-1)^{k} \int_{m}^{\infty}\left(\frac{u}{k+u}\right)^{m} \frac{k}{(k+u)^{2}} \frac{([u]+k+1) !}{[u] ! k !} \Delta^{k} \mu_{[u]} d u$.

Next we observe that

$$
\mu_{m}=\sum_{p=m}^{\infty} \frac{(p+k-m-1) !}{(k-1) !(p-m) !}(-1)^{k} \Delta^{k} \mu_{p} .
$$

This result is easily proved by induction, making use of (22.1). 
Now write the summation (22.4) as an integral as follows:

$$
\mu_{m}=(-1)^{k} \int_{m}^{\infty} \frac{([u]+k-m-1) !}{(k-1) !([u]-m) !} \Delta^{k} \mu_{[u]} d u .
$$

Then

$$
\begin{aligned}
H_{k}(m)-\mu_{m}= & (-1)^{k} \int_{m}^{\infty} \Delta^{k} \mu_{[u]}\left\{\left(\frac{u}{k+u}\right)^{m} \frac{k}{(k+u)^{2}} \frac{([u]+k+1) !}{[u] ! k !}\right. \\
& \left.-\frac{([u]+k-m-1) !}{(k-1) !([u]-m) !}\right\} d u .
\end{aligned}
$$

By virtue of (22.1)

$$
\begin{aligned}
\left|\boldsymbol{H}_{k}(m)-\mu_{m}\right| \leqq & M \int_{m}^{\infty}\left(\frac{u}{k+u}\right)^{m} \frac{k}{(k+u)^{2}} \mid 1-\frac{[u]}{u} \frac{[u]-1}{u} \ldots \\
& \cdot \frac{[u]-m+1}{u} \frac{k+u}{[u]+k+1} \frac{k+u}{[u]+k} \cdots \frac{k+u}{[u]+k-m} \mid d u .
\end{aligned}
$$

But

$$
\frac{u+k}{[u]+k+j}=1+\frac{u-[u]-j}{[u]+k+j}=1+o\left(\frac{1}{k}\right) \quad(k \rightarrow \infty)
$$

uniformly for $m \leqq u<\infty$. Also

$$
\left|\frac{[u]-j}{u}-1\right|=\left|\frac{[u]-u-j}{u}\right| \leqq \frac{N}{u} \quad(j=0,1,2, \cdots, m-1),
$$

where $N$ is a constant independent of $u$ and of $j$. Hence for $m \geqq 1$ we have

$$
\begin{aligned}
H_{k}(m)-\mu_{m} & =O\left\{\int_{m}^{\infty} \frac{k}{(k+u)^{2}} \frac{1}{k} d u\right\} & & (k \rightarrow \infty) \\
& =O\left(\frac{1}{k}\right) & & (k \rightarrow \infty),
\end{aligned}
$$

so that

$$
\lim _{k \rightarrow \infty} H_{k}(m)=\mu_{m} \quad(m=1,2,3, \cdots) .
$$

For $m=0$ we have from (22.3)

$$
\begin{aligned}
H_{k}(0) & =(-1)^{k} \sum_{p=0}^{\infty} \frac{(p+k+1) !}{p ! k !} \Delta^{k} \mu_{p} \int_{p}^{p+1} \frac{k}{(k+u)^{2}} d u \\
& =(-1)^{k} \sum_{p=0}^{\infty} \frac{(p+k-1) !}{(k-1) ! p !} \Delta^{k} \mu_{p} .
\end{aligned}
$$


But reference to (22.4) shows that this is $\mu_{0}$, and (22.6) holds also for $m=0$.

It remains to show that

$$
\lim _{k \rightarrow \infty} J_{k}(m)=0 \quad(m=0,1,2, \cdots) .
$$

By virtue of (22.1) we have

$$
\left|J_{k}(m)\right| \leqq \int_{0}^{m /(k+m)} t^{m} M d t \leqq \frac{M m}{k+m},
$$

so that (22.7) is evident. The theorem is thus established.

We turn next to a corresponding result for Stieltjes integrals.

TheOREM 41. If

$$
\left|\sum_{p=m}^{\infty} \frac{(p+k) !}{p ! k !} \Delta^{k+1} \mu_{p}\right|<M \quad(m=0,1,2, \cdots ; k=0,1,2, \cdots),
$$

then

$$
\mu_{m}-\mu_{\infty}=\lim _{k \rightarrow \infty} \int_{0}^{1} t^{m} d S_{k, t}\left\{\mu_{n}\right\} \quad(m=0,1,2, \cdots),
$$

provided $S_{k, 1}\left\{\mu_{n}\right\}$ is defined as $-\mu_{\infty}$.

To prove this result we rewrite the sum (22.8) as follows:

$$
(-1)^{k} \sum_{p=m}^{\infty} \frac{(p+k-1) !}{p !(k-1) !} \Delta^{k} \mu_{p}=(-1)^{k} \sum_{p=m}^{\infty}\left\{\frac{(p+k) !}{k ! p !}-\frac{(p+k-1) !}{k !(p-1) !}\right\} \Delta^{k} \mu_{p} \text {. }
$$

Now

$$
\begin{aligned}
(-1)^{k} \sum_{p=m}^{q} \frac{(p+k-1) !}{p !(k-1) !} \Delta^{k} \mu_{p}= & (-1)^{k} \sum_{p=m}^{q} \frac{(p+k) !}{k ! p !} \Delta^{k} \mu_{p} \\
& -(-1)^{k} \sum_{p=m}^{q} \frac{(p+k-1) !}{k !(p-1) !} \Delta^{k} \mu_{p}
\end{aligned}
$$

for any integer $q>m$. Changing the summation variable in the last of these sums we have

$$
\begin{aligned}
&(-1)^{k} \sum_{p=m}^{q} \frac{(p+k-1) !}{p !(k-1) !} \Delta^{k} \mu_{p} \\
& \quad=(-1)^{k} \sum_{p=m}^{q} \frac{(p+k) !}{k ! p !} \Delta^{k} \mu_{p}-(-1)^{k} \sum_{p=m+1}^{q+1} \frac{(p+k) !}{k ! p !} \Delta^{k} \mu_{p+1}
\end{aligned}
$$




$$
\begin{aligned}
=(-1)^{k+1} & \sum_{p-m+1}^{q} \frac{(p+k) !}{k ! p !} \Delta^{k+1} \mu_{p} \\
& +(-1)^{k} \frac{(m+k) !}{k ! m !} \Delta^{k} \mu_{m}-(-1)^{k} \frac{(q+k+1) !}{k !(q+1) !} \Delta^{k} \mu_{q+2} .
\end{aligned}
$$

Now let $q$ become infinite. Since the summations on both sides of the equation approach limits by hypothesis, it follows that

$$
\lim _{q \rightarrow \infty} \frac{(q+k) !}{k ! q !} \Delta^{k} \mu_{q+1}
$$

exists $(k=0,1,2, \cdots)$. We can show, in fact, that all these limits are zero except perhaps that corresponding to $k=0$. For, suppose

$$
\lim _{q \rightarrow \infty} q \Delta \mu_{q}=B>0 \text {. }
$$

Then for $q \geqq q_{0}$ we have

$$
q \Delta \mu_{q}>B / 2
$$

and

$$
\mu_{q_{0}+n+1}-\mu_{q_{0}}=\sum_{q=q_{0}}^{q_{0}+n} \Delta \mu_{q}>\frac{B}{2} \sum_{q=q_{0}}^{q_{0}+n} \frac{1}{q} .
$$

As $n$ becomes infinite the right-hand side of this inequality becomes positively infinite, so that $\mu_{q}$ can approach no limit as $q$ becomes infinite, contrary to the fact established above. If $B<0$ we deduce a contradiction by applying the foregoing proof to the sequence $\left\{-\mu_{q}\right\}$. Proceeding by induction suppose it has been established that

$$
\lim _{q \rightarrow \infty} \frac{(q+k) !}{k ! q !} \Delta^{k} \mu_{q+1}=0 ;
$$

let us show the same to be true when $k$ is replaced by $k+1$. We have

$$
\text { 9) }(q+1) \Delta\left\{\frac{(q+k) !}{k ! q !} \Delta^{k} \mu_{q+1}\right\}=\frac{(q+k+1) !}{k ! q !} \Delta^{k+1} \mu_{q+1}+\frac{(q+k) !}{q !(k+1) !} \Delta^{k} \mu_{q+1} \text {. }
$$

By assumption the right-hand side approaches a limit. It follows that

$$
q \Delta\left\{\frac{(q+k) !}{k ! q !} \Delta^{k} \mu_{q+1}\right\}
$$

approaches the same limit. Since

$$
\frac{(q+k) !}{k ! q !} \Delta^{k} \mu_{q+1}
$$


also approaches a limit we may apply the argument used for the case $k=0$ to the sequence (22.10). It follows that the limit of this sequence must be zero and hence by (22.9) that

$$
\lim _{q \rightarrow \infty} \frac{(q+k+1) !}{(k+1) ! q !} \Delta^{k+1} \mu_{q+1}=0 .
$$

We showed in $\$ 21$ that

$$
\frac{(n+k+1) !}{n ! k !}(-1)^{k} \Delta^{k}\left\{\frac{\mu_{n+1}}{n+1}\right\}
$$

provided that

$$
=\mu_{\infty}+(-1)^{k+1} \sum_{i=n}^{\infty} \frac{(i+k+1) !}{k !(i+1) !} \Delta^{k+1} \mu_{i+1}
$$

$$
\lim _{i \rightarrow \infty} \frac{(i+k+1) !}{(k+1) ! i !} \Delta^{k+1} \mu_{i+1}=0 .
$$

But we have just established this latter result. Then by (22.2) and (22.8) we have

$$
\left|\frac{(n+k+1) !}{n ! k !} \Delta^{k}\left\{\frac{\mu_{n+1}}{n+1}\right\}\right|<\left|\mu_{\infty}\right|+M .
$$

By Theorem 40 it follows that

$$
\frac{\mu_{m+1}}{m+1}=\lim _{k \rightarrow \infty} \int_{0}^{1} t^{m} L_{k, t}\left\{\frac{\mu_{n+1}}{n+1}\right\} d t
$$

By (22.11) we see that

Hence

$$
L_{k, \ell}\left\{\frac{\mu_{n+1}}{n+1}\right\}=-S_{k, t}\left\{\mu_{n}\right\}
$$

$$
\begin{aligned}
\frac{\mu_{m+1}}{m+1} & =-\lim _{k \rightarrow \infty} \int_{0}^{1} t^{m} S_{k, t}\left\{\mu_{n}\right\} d t \\
& =-\left.\lim _{k \rightarrow \infty} \frac{t^{m+1}}{m+1} S_{k, t}\left\{\mu_{n}\right\}\right|_{t=0} ^{t=1}+\lim _{k \rightarrow \infty} \frac{1}{m+1} \int_{0}^{1} t^{m+1} d S_{k, t}\left\{\mu_{n}\right\}, \\
\mu_{m+1} & =\lim _{k \rightarrow \infty} \int_{0}^{1} t^{m+1} d S_{k, t}\left\{\mu_{n}\right\}+\mu_{\infty} .
\end{aligned}
$$

This completes the proof of the theorem. 
23. Hausdorff's theorem. Just as our inversion operator enabled us to give a proof of Bernstein's theorem so will the present inversion operator enable us to give a proof of a familiar theorem of Hausdorff.

THEOREM 42. A necessary and sufficient condition that the equations

$$
\mu_{n}=\int_{0}^{1} t^{n} d \alpha(t) \quad(n=0,1,2, \cdots)
$$

should have a bounded non-decreasing solution $\alpha(t)$ is that the sequence $\left\{\mu_{n}\right\}$ should be completely monotonic:

$$
(-1)^{k} \Delta^{k} \mu_{n} \geqq 0 \quad(k=0,1,2, \cdots ; n=0,1,2, \cdots) .
$$

The necessity of the condition follows at once from the equation

$$
(-1)^{k} \Delta^{k} \mu_{n}=\int_{0}^{1} t^{n}(1-t)^{k} d \alpha(t)
$$

To prove the sufficiency apply the operator $S_{t}$ to the sequence $\left\{\mu_{n}\right\}$. We must first show that $S_{k, t}\left\{\mu_{n}\right\}$ exists. To do this we show that for a completely monotonic sequence $\mu_{n}$ we have

$$
\lim _{n \rightarrow \infty} \frac{(n+k) !}{n ! k !} \Delta^{k} \mu_{n+1}=c_{k} \quad(k=0,1,2, \cdots) .
$$

We use induction. The result is immediate for $k=0$, for the sequence $\left\{\mu_{n}\right\}$ is non-negative non-increasing. Next form the sequence

$$
\nu_{n}=\mu_{n+1}-(n+1) \Delta \mu_{n+1} \quad(n=0,1,2, \cdots) .
$$

This is also a non-negative sequence. Moreover,

$$
\begin{aligned}
\Delta \nu_{n} & =\Delta \mu_{n+1}-(n+2) \Delta^{2} \mu_{n+1}-\Delta \mu_{n+1} \\
& =-(n+2) \Delta^{2} \mu_{n+1} .
\end{aligned}
$$

This is not greater than zero, so that the sequence $\left\{\nu_{n}\right\}$ is also non-increasing, and must therefore approach a limit. Since $\mu_{n+1}$ has a limit, the same must be true of $(n+1) \Delta \mu_{n+1}$. We proceed by induction. Suppose that (23.1) has been established for $k<m$. Form the sequence

$$
\nu_{n}=\mu_{n+1}-(n+1) \Delta \mu_{n+1}+\frac{(n+1)(n+2)}{2 !} \Delta^{2} \mu_{n+1}-\cdots
$$

$$
+(-1)^{m} \frac{(n+m) !}{m ! n !} \Delta^{m} \mu_{n+1} .
$$


Simple computation gives

$$
\Delta \nu_{n}=(-1)^{m} \frac{(n+m+1) !}{m !(n+1) !} \Delta^{m+1} \mu_{n+1} \leqq 0 .
$$

It follows that $\nu_{n}$ is a non-negative, non-increasing sequence and hence must approach a limit. Thus every term in (23.2) except the last is known to have a limit. It follows that (23.1) holds for $k=m$, and the induction is complete.

In the proof of Theorem 41 we showed that the existence of the limits $c_{k}$ implied that they were all zero except perhaps $c_{0}$. This, in turn, implies the convergence of the series

$$
(-1)^{k+1} \sum_{i=m}^{\infty} \frac{(i+k+1) !}{k !(i+1) !} \Delta^{k+1} \mu_{i+1} .
$$

Moreover

$$
\begin{aligned}
\sum_{i=n}^{\infty} \frac{(i+k+1) !}{k ! i !}(-1)^{k+1} \Delta^{k+1} \mu_{i+1} & \\
= & \sum_{i=n}^{\infty} \frac{(i+k+2) !}{(k+1) !(i+1) !}(-1)^{k+2} \Delta^{k+2} \mu_{i+1} .
\end{aligned}
$$

The derivation of this formula involved (23.1) as well as the convergence of the series. But the series on the left surely converges for $k=0$ since the limit of $\mu_{n}$ exists. Hence by induction we see that (23.3) converges for all $k$.

Having verified that the operator $S_{k, t}\left\{\mu_{n}\right\}$ exists we prove next that it defines a non-decreasing function. We have

$$
S_{k, t}\left\{\mu_{n}\right\}=-\mu_{\infty}-\sum_{i=n}^{\infty} \frac{(i+k+1) !}{k !(i+1) !}(-1)^{k+1} \Delta^{k+1} \mu_{i+1}, \quad n=\left[\frac{k t}{1-t}\right] .
$$

As $t$ increases $n$ is non-decreasing, so that

$$
\sum_{i=n}^{\infty} \frac{(i+k+1) !}{k !(i+1) !}(-1)^{k+1} \Delta^{k+1} \mu_{i+1}
$$

is surely non-increasing. That is, $S_{k, t}\left\{\mu_{n}\right\}$ is non-decreasing. The same must be true of $S_{t}\left\{\mu_{n}\right\}$. Clearly

$$
\begin{aligned}
-S_{k, t}\left\{\mu_{n}\right\} & \leqq-S_{k, 0}\left\{\mu_{n}\right\}=\mu_{\infty}+(-1)^{k+1} \sum_{i=0}^{\infty} \frac{(i+k+1) !}{k !(i+1) !} \Delta^{k+1} \mu_{i+1} \\
& \leqq \mu_{\infty}+(-1)^{k+1} \sum_{i=0}^{\infty} \frac{(i+k+1) !}{k !(i+1) !} \Delta^{k+1} \mu_{i+1}+(-1)^{k+1} \Delta^{k+1} \mu_{0} \\
& =\mu_{\infty}+\sum_{i=0}^{\infty} \frac{(i+k) !}{k ! i !}(-1)^{k+1} \Delta^{k+1} \mu_{i}=\mu_{0} .
\end{aligned}
$$


This latter result is certainly exact for $k=0$ and is easily seen to be true in general by induction using (23.4). Hence the inequalities (22.8) are satisfied by the given completely monotonic sequence $\left\{\mu_{n}\right\}$. Hence by Theorem 41

$$
\mu_{m}-\mu_{\infty}=\int_{0}^{1} t^{m} d S_{k, 8}\left\{\mu_{n}\right\}
$$

Now we employ Helly's theorem precisely as we did in the proof of Theorem 17 to select from the bounded sequence of non-decreasing functions $S_{k, t}\left\{\mu_{n}\right\}$ a sub-set which approaches a non-decreasing function $\beta(t)$. As in that proof we see that

$$
\mu_{m}-\mu_{\infty}=\int_{0}^{1} t^{m} d \beta(t) .
$$

Here $\beta(1)=-\mu_{\infty}$. We define $\alpha(t)$ as follows:

$$
\begin{array}{ll}
\alpha(t)=\beta(t) & (0 \leqq t<1), \\
\alpha(1) & =0 .
\end{array}
$$

The function $\alpha(t)$ remains non-decreasing since $-\mu_{\infty} \leqq 0$ and

$$
\mu_{m}=\int_{0}^{1} t^{m} d \alpha(t) \quad(m=0,1,2, \cdots) .
$$

This completes the proof of the theorem. $\dagger$

The present methods are powerful in the discussion of what sequences are moment sequences. We use them to prove one further result of Hausdorff.

† Compare F. Hausdorff, Momentprobleme für ein endliches Intervall, Mathematische Zeitschrift, vol. 16 (1923), pp. 220-248. It is of interest to compare Hausdorff's method of approximation to the function $\alpha(t)$ with our own. His $k$ th approximating function $\chi_{k}(t)$ is a step-function which vanishes at $t=0$ and has a jump of amount

$$
(-1)^{k-n}\left(\begin{array}{l}
k \\
n
\end{array}\right) \Delta^{k-n} \mu^{n}
$$

at the point $n / k(n=0,1,2, \cdots, k)$. Our $k$ th approximating function, $S_{k, t}\left\{\mu^{n}\right\}$, is also a step-function vanishing at the origin and with jump of amount

$$
(-1)^{k+1}\left(\begin{array}{c}
n+k \\
n
\end{array}\right) \Delta^{k+1} \mu^{n}
$$

at the points $n /(n+k)(n=0,1,2, \cdots)$. Thus the function $\chi_{k}(t)$ has $(k+1)$ jumps which depend on differences of order less than or equal to $k$. On the other hand the function $S_{k, 2}\left\{\mu^{n}\right\}$ has infinitely many jumps which cluster about the point $t=1$, the amount of the jumps depending on the $(k+1)$ th differences only. We note further that the function $L_{k, t}\left\{\mu^{n}\right\}$ is also a step-function with jumps at the same points $n /(n+k)$, the amounts of the jumps depending on differences of order $k$ only. 
Theorem 43. A necessary and sufficient condition that

$$
\mu_{n}=\int_{0}^{1} t^{n} d \alpha(t) \quad(n=0,1,2, \cdots)
$$

where $\alpha(t)$ is of bounded variation in $(0,1)$ is that for a suitable constant $M$

$$
\sum_{p=m}^{\infty} \frac{(p .+k) !}{p ! k !}\left|\Delta^{k+1} \mu_{p}\right|<M \quad(m=0,1,2, \cdots ; k=0,1,2, \cdots) .
$$

We first prove the necessity of the condition. Assume that $\mu_{n}$ is defined by (23.5) with $\alpha(t)$ of bounded variation in $(0,1)$. Denote by $V(t)$ the total variation of $\alpha(x)$ in the interval $0 \leqq x \leqq t$. Then by Theorem 42 the sequence

$$
\nu_{n}=\int_{0}^{1} t^{n} d V(t) \quad(n=0,1,2, \cdots)
$$

is completely monotonic. Hence

$$
\begin{gathered}
\left|\Delta^{k+1} \mu_{p}\right| \leqq(-1)^{k+1} \Delta^{k+1} \nu_{p}, \\
\sum_{p=n}^{\infty} \frac{(p+k) !}{p ! k !}\left|\Delta^{k+1} \mu_{p}\right| \leqq \sum_{p=0}^{\infty} \frac{(p+k) !}{p ! k !}(-1)^{k+1} \Delta^{k+1} \nu_{p}=\nu_{0}-\nu_{\infty}=M .
\end{gathered}
$$

This completes the proof of the necessity.

We turn to the sufficiency of the condition. By condition (23.6) we see at once that Theorem 41 is applicable to the sequence $\left\{\mu_{n}\right\}$ so that

$$
\mu_{m}-\mu_{\infty}=\lim _{k \rightarrow \infty} \int_{0}^{1} t^{m} d S_{k, t}\left\{\mu_{n}\right\} .
$$

Simple computation shows that the function $S_{k, t}\left\{\mu_{n}\right\}$ is a function of bounded variation and that its total variation in the interval $(0,1)$ is not greater than $M$.

We now employ the theorem of Helly used in the proof of Theorem 19 to select a convergent sequence of functions from the sequence $S_{k, t}\left\{\mu_{n}\right\}$. The limit function $\alpha(t)$ will itself be a function of bounded variation and by (23.7) we have

$$
\mu_{m}-\mu_{\infty}=\int_{0}^{1} t^{m} d \alpha(t)
$$

If $\alpha(t)$ is redefined at $t=1$ to be zero there, we have

$$
\mu_{m}=\int_{0}^{1} t^{m} d \alpha(t) \quad(m=0,1,2 \ldots
$$


and the proof of the theorem is complete.

24. The changes of sign in a moment sequence. The operators $S$ and $L$ are useful in discussing the changes of sign in a sequence

$$
\mu_{n}=\int_{0}^{1} t^{n} d \alpha(t) \quad(n=0,1,2, \cdots)
$$

in terms of the changes of trend in the function $\alpha(t)$ or in terms of the changes of sign in the derivative $\alpha^{\prime}(t)$, if it exists. We begin by proving

THEOREM 44. If the function $\alpha(t)$ has $m$ changes of trend in the interval $(0,1)$ then the sequence

$$
\mu_{n}=\int_{0}^{1} t^{n} d \alpha(t) \quad(n=0,1,2, \cdots)
$$

can have at most $m$ changes of sign.

For, set

$$
\begin{aligned}
& \mu(x)=\int_{0}^{1} t^{x} d \alpha(t)=\int_{0}^{\infty} e^{-x t} d \beta(t), \\
& \beta(t)=-\alpha\left(e^{-t}\right) .
\end{aligned}
$$

Since $\mu(x)$ is continuous (analytic in fact), and since $\mu(n)=\mu_{n}$, the function $\mu(x)$ would have more than $m$ zeros if the sequence (24.1) had more than $m$ changes of sign. But this is impossible by Theorem 22. We next establish

THEOREM 45. If the function $\alpha(t)$ is a normalized function of bounded variation with $\alpha(1)=\alpha(1-)=0$ and with $m$ changes of trend in $(0,1)$, then the sequence

$$
(-1)^{k} \Delta^{k} \mu_{n}=\int_{0}^{1} t^{n}(1-t)^{k} d \alpha(t) \quad(n=0,1,2, \cdots)
$$

has exactly $m$ changes of sign for all $k$ sufficiently large.

We prove exactly as in the proof of Theorem 23 that corresponding to two adjoining intervals $\left(t_{i-1}, t_{i}\right),\left(t_{i}, t_{i+1}\right)$ of the intervals referred to in the definition of number of changes of trend (in the first of which $\alpha(t)$ is increasing, in the second of which, decreasing) there are three points $\xi, \eta, \zeta$ such that

$$
\alpha(\xi)<\alpha(\eta)>\alpha(\zeta) \quad\left(t_{i-1} \leqq \xi<\eta<\zeta \leqq t_{i+1}\right) .
$$

We then determine $k_{0}$ so large that for $k>k_{0}$ we have

$$
\left[\frac{\xi k}{1-\xi}\right]<\left[\frac{k \eta}{1-\eta}\right]<\left[\frac{k \zeta}{1-\zeta}\right]
$$


and such that

$$
S_{k, \xi}\left\{\mu_{n}\right\}<S_{k, \eta}\left\{\mu_{n}\right\}>S_{k, 5}\left\{\mu_{n}\right\}
$$

for $k>k_{0}$. This is possible by Theorem 37 . Hence

$$
\begin{array}{ll}
(-1)^{k+1} \sum_{i=n}^{n \eta-1} \frac{(i+k+1) !}{k !(i+1) !} \Delta^{k+1} \mu_{i+1}>0, & n_{\xi}=\left[\frac{k \xi}{1-\xi}\right], \quad n_{\eta}=\left[\frac{k \eta}{1-\eta}\right], \\
(-1)^{k+1} \sum_{i=n \eta}^{n \zeta} \frac{(i+k+1) !}{k !(i+1) !} \Delta^{k+1} \mu_{i+1}<0, & n_{\zeta}=\left[\frac{k \zeta}{1-\zeta}\right],
\end{array}
$$

There is at least one term in each series, so that there must be at least one change of sign in the sequence $\left\{\Delta^{k+1} \mu_{n}\right\}(n=0,1,2, \cdots)$ between the terms $n_{\xi}$ and $n_{\xi}$. We are thus able to show as in the proof of Theorem 23 that the sequence (24.1) has at least $m$ changes of sign for $k$ sufficiently large. That it can not have more follows from Theorem 44 .

COROLLARY 1. If the function $\alpha(t)$ has a maximum (minimum) at a point $t=t_{0}$, then for $k$ sufficiently large the sequence

$$
(-1)^{k} \Delta^{k} \mu_{n}=\int_{0}^{1} t^{n}(1-t)^{k} d \alpha(t) \quad(n=0,1,2, \cdots)
$$

will have a change of sign at a term $n=n_{k}$ such that

$$
\lim _{k \rightarrow \infty} \frac{n_{k}}{n_{k}+k}=t_{0} \text {. }
$$

For, if $\epsilon$ is an arbitrary positive number we must show that a number $k_{0}$ exists such that the sequence $\Delta^{k} \mu_{n}$ has a change of sign at $n=n_{k}$, where

$$
\left|\frac{n_{k}}{n_{k}+k}-t_{0}\right|<\epsilon .
$$

As in the proof of the theorem we see that for $k$ sufficiently large the sequence changes sign between the terms with indices $n_{\xi}$ and $n_{\zeta}$ where now

$$
\xi=t_{0}-(\epsilon / 2), \eta=t_{0}+(\epsilon / 2) .
$$

Suppose that a change of sign occurs at the term $n=n_{k}$. Then

Hence

$$
k \xi /(1-\xi)-1<n_{\xi}<n_{k}<n_{\xi} \leqq k \zeta /(1-\zeta) .
$$

$$
\begin{gathered}
\frac{n_{k}}{n_{k}+k}<\zeta<t_{0}+\epsilon, \\
\xi<\frac{n_{k}+1}{n_{k}+k+1}, \xi-\frac{k}{\left(n_{k}+k\right)\left(n_{k}+k+1\right)}<\frac{n_{k}}{n_{k}+k} .
\end{gathered}
$$


Since the left-hand side of this last inequality approaches $\xi$ as $k$ becomes infinite we can find a number $k_{0}$ so large that

$$
t_{0}-\epsilon=\xi-\frac{\epsilon}{2}<\frac{n_{k}}{n_{k}+k} \quad\left(k>k_{0}\right) .
$$

This proves the corollary.

COROLLARY 2. If the function $\phi(t)$ is integrable in $(0,1)$ and has a change of sign at $t_{0}$ between 0 and 1 , then the sequence

$$
(-1)^{k} \Delta^{k} \mu_{n}=\int_{0}^{1} t^{n}(1-t)^{k} \phi(t) d t
$$

has a change of sign at a term with index $n_{k}$ such that

$$
\lim _{k \rightarrow \infty} \frac{n_{k}}{n_{k}+k}=t_{0} \text {. }
$$

One proves this by Corollary 1 , setting

$$
\alpha(t)=\int_{t}^{1} \phi(u) d u
$$

and observing that $\alpha(t)$ has a maximum or minimum at $t_{0}$. This result is a generalization of a theorem of Fekete cited in the introduction. Fekete considered only functions $\phi(t)$ which are continuous.

25. Complex variable. Hitherto we have considered only real moment sequences. It is natural to inquire whether our inversion operator is still valid for complex sequences. Let

$$
\mu_{n}=\int_{0}^{1} t^{n} \phi(t) d t
$$

where now $\phi(t)$ is a complex function. We shall continue to take the path of integration as the real axis. By breaking $\phi(t)$ into real and imaginary parts we could easily show that

$$
L_{t}\left\{\mu_{n}\right\}=\phi(t)
$$

for all real $t$ between 0 and 1 . However, we wish also to obtain an inversion formula which will be valid for complex $t$. The operator $L$ becomes meaningless for complex $t$ since its definition involves the greatest integer contained in $k t /(1-t)$. We may define an operator $L_{t}^{*}$ which is applicable to any sequence defined by (25.1) as follows. 
Definition. An operator $L_{t}^{*}\left\{\mu_{n}\right\}$ is defined by the equations

$$
\begin{gathered}
L_{k, t}^{*}\left\{\mu_{n}\right\}=\frac{\Gamma(\omega+k+2)}{\Gamma(\omega+1) \Gamma(k+1)}(-1)^{k} \Delta^{k} \mu_{\omega}, \quad \omega=\frac{k t}{1-t}, \\
L_{t}^{*}\left\{\mu_{n}\right\}=\lim _{k \rightarrow \infty} L_{k, t}\left\{\mu_{n}\right\} .
\end{gathered}
$$

In this definition the factor $\Delta^{k} \mu_{\omega}$ is taken to mean

$$
\Delta^{k} \mu_{\omega}=(-1)^{k} \int_{0}^{1} u^{\omega}(1-u)^{k} \phi(u) d u
$$

when $t$ is not an integer. We shall be able to show that this operator inverts the sequence (25.1) not only for all real $t$ between 0 and 1 but for all $t$ in a circle of unit diameter with center at $t=\frac{1}{2}$. In fact we prove

THEOREM 46. If the function $\phi(u)$ is analytic in the circle

$$
\left|u-\frac{1}{2}\right|<\frac{1}{2}
$$

and if

$$
\mu_{n}=\int_{0}^{1} u^{n} \phi(u) d u
$$

then for any $t$ in that circle

$$
L_{t}^{*}\left\{\mu_{n}\right\}=\phi(t) .
$$

If $t_{0}$ is in the circle $C$ described in the theorem, then

$$
R\left(t_{0} /\left(1-t_{0}\right)\right)>0,
$$

where the symbol $R$ denotes "the real part of." Hence the integral

$$
\int_{0}^{1} u^{k \omega_{0}}(1-u)^{k} \phi(u) d u, \omega_{0}=t_{0} /\left(1-t_{0}\right)
$$

converges for all positive $k$, and we have

$$
L_{k, t_{0}}^{*}\left\{\mu_{n}\right\}=\int_{0}^{1} u^{k \omega_{0}}(1-u)^{k} \phi(u) d u / \int_{0}^{1} u^{k \omega_{0}}(1-u)^{k} d u .
$$

By the function $u^{k \omega_{0}}$ we mean $\exp \left(k \omega_{0} \log u\right)$, the real determination of the logarithm being taken. To evaluate the limit of $L_{k}{ }^{*} t_{0}\left\{\mu_{n}\right\}$ as $k$ becomes infinite we employ the method of $O$. Perron. $†$ To make use of this method we

† O. Perron, Über die näherungsweise Berechnung von Funktionen grosser Zahlen, Sitzungsberichte der Akademie der Wissenschaften zu München, mathematisch-physikalischen Klasse, vol. 7 (1917), pp. 191-219. 
must alter the path of integration in both integrals (25.2) so as to make it pass through the point $t_{0}$.

Set

$$
g(u)=u^{\omega_{0}}(1-u)=\exp \left\{\omega_{0} \log u+\log (1-u)\right\},
$$

where $u$ is in the circle $C$ and where that determination of the logarithm is taken which is real when $u$ is real. It is easily verified that $g^{\prime}\left(t_{0}\right)=0$. If we set

$$
h(v)=\left(\frac{v+t_{0}}{t_{0}}\right)^{\omega_{0}}\left(\frac{1-v-t_{0}}{1-t_{0}}\right)
$$

the power series development of $\log h(v)$ begins as follows:

$$
\log h(v)=-\frac{v^{2}}{2 t_{0}\left(1-t_{0}\right)^{2}}+\cdots .
$$

Now define $r$ and $\beta$ by the equation

$$
r e^{i \beta}=-1 /\left\{2 t_{0}\left(1-t_{0}\right)^{2}\right\}
$$

and apply Perron's result. We obtain

$$
\begin{array}{ll}
\int_{0}^{1} u^{k \omega_{0}}(1-u)^{k} \phi(u) d u \sim(k r)^{-1 / 2} i e^{i \beta / 2}(\pi)^{1 / 2} \phi\left(t_{0}\right), & \\
\int_{0}^{1} u^{k \omega_{0}}(1-u)^{k} d u \sim(k r)^{-1 / 2} i e^{i \beta / 2}(\pi)^{1 / 2} & (k \rightarrow \infty) .
\end{array}
$$

It follows that

$$
\lim _{k \rightarrow \infty} L_{k}^{*}\left\{\mu_{n}\right\}=\phi\left(t_{0}\right) .
$$

One of the hypotheses assumed by Perron, when stated for the case in hand, was that it should be possible to pass a curve from $u=0$ to $u=1$ in the circle $C$ such that for all points of the curve except $u=t_{0}$

$$
|g(u)|<\left|g\left(t_{0}\right)\right| \text {. }
$$

To establish the existence of such a curve consider the level lines

$$
|g(u)|=\left|g\left(t_{0}\right)\right| \text {. }
$$

We can show that they consist of two curves intersecting only at $t_{0}$ which divide the circle into four parts. In two of these parts

$$
|g(u)|<\left|g\left(t_{0}\right)\right|
$$

and in two

$$
|g(u)|>\left|g\left(t_{0}\right)\right| \text {. }
$$

Without setting down the details of the proof we point out that it will 
be quite sufficient to show that, on the boundary of $C,|g(u)|$ has only two relative maxima and only two minima ( $u=0$ and $u=1$ ).

If $u=\rho e^{i \theta}$, simple computation shows that on the boundary of $C$, where $\rho=\cos \theta$,

$$
|g(u)|=(\cos \theta)^{a} e^{-b \theta}(1-\cos \theta)=\gamma(\theta) .
$$

Here $a$ and $b$ are the real and imaginary parts of $\omega_{0}$ respectively. It will be sufficient to show that the logarithmic derivative of $\gamma(\theta)$ vanishes just twice for $-\pi / 2<\theta<\pi / 2$. But

$$
\frac{\gamma^{\prime}(\theta)}{\gamma(\theta)}=-a \tan \theta-b+\cot (\theta / 2) .
$$

Since $a>0$ the curve

$$
y=a \tan x+b
$$

consists of two branches, one descending from $+\infty$ to $-\infty$ as $x$ varies from $-\pi / 2$ to 0 , the other descending from $+\infty$ to $-\infty$ as $x$ varies from 0 to $\pi / 2$. On the other hand the curve

$$
y=\cot (\theta / 2)
$$

consists of a single branch ascending from $-\infty$ to $+\infty$ as $x$ varies from $-\pi / 2$ to $\pi / 2$. These two curves can intersect in only two points, the points where $\gamma(\theta)$ is maximum. One of these points clearly lies between $-\pi / 2$ and 0 , the other between 0 and $\pi / 2$. The level lines through $t=t_{0}$ must then cut the circle at only four points, thus dividing the circle into four parts as described above. This completes the proof of the theorem.

HARVARD UNIVERSITY,

Cambridge, Mass. 